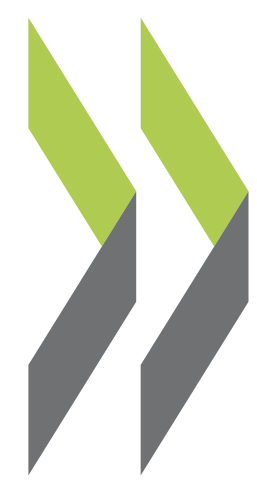

Documents de travail de l'OCDE sur la gouvernance publique No. 12

La gestion du changement dans l'administration des pays de l'OCDE : Un Oscar Huerta Melchor premier aperçu général 
Documents de travail sur la gouvernance publique $\mathrm{n}^{\circ} 12$

\section{La gestion du changement dans l'administration des pays de l'OCDE}

un premier aperçu général

Oscar Huerta Melchor

Juin 2008 



\begin{abstract}
AVANT-PROPOS
Dans son programme de travail et son budget pour 2007-2008, la Direction de la gouvernance publique et du développement territorial de l'OCDE (GOV) prévoit la réalisation d'une étude sur la gestion du changement dans l'administration. L'objectif est d'étudier les différentes manières dont les pays de I'OCDE gèrent le changement produit par l'introduction de grandes mesures de réforme destinées à transformer la culture administrative du service public afin de le rendre plus performant et efficace.

Afin de préparer le terrain avant l'étude, GOV a entrepris un examen des travaux publiés et a rédigé un document préliminaire soumis pour examen à la réunion du Groupe de travail sur l'emploi et la gestion publics de l'OCDE en novembre 2007. Six pays de l'OCDE ont présenté leur expérience en matière de gestion du changement: la Finlande, la France, I'Italie, le Portugal, l'Espagne et la Suisse. Ce document tire des conclusions préliminaires à étudier et examiner plus avant, qui pourraient être intégrées à un futur programme de travail.

Ce projet a été dirigé par Oscar Huerta Melchor (Secrétariat de l'OCDE) et contrôlé par Elsa Pilichowski (Secrétariat de l'OCDE) sous la supervision de Barry Anderson (Secrétariat de l'OCDE). Les six études de cas sont des versions révisées des rapports présentés à la réunion du Groupe de travail sur l'emploi et la gestion publics en 2007 par les agents publics suivants des pays Membres de l'OCDE :
\end{abstract}

- Finlande : M. Veikko Liuksia, directeur de la politique du personnel au Ministère des finances, M. Asko Lindqvist, responsable du programme Finwin ;

- France : Mlle Françoise Waintrop, Direction générale de l'administration et de la fonction publique ;

- Italie : M. Francesco Verbaro, Bureau du Premier ministre, directeur du bureau de la fonction publique ;

- Portugal: Mlle Joana Ramos, Direction générale de l'administration et de la fonction publique, Mlle Teresa Ganhão, Direction de l'administration publique ;

- Espagne: M. Emilio Viciana Duro, Ministère des administrations publiques, Mlle Isabel Hernández, Ministère des administrations publiques ;

- Suisse : M. Thierry Borel, Département fédéral des finances. 


\section{SYNTHÈSE}

La réforme du secteur public est une question complexe. Les pays Membres et non membres de l'OCDE ont de plus en plus de difficultés à concrétiser le changement. II ne suffit plus désormais d'adopter des réformes novatrices pour répondre aux demandes de la société ; les gouvernements doivent accompagner leurs propositions de réforme d'une stratégie de gestion du changement. La gestion du changement conditionne, dans une large mesure, la réussite ou l'échec d'une action de réforme.

Le présent document fait valoir que les concepts de réforme et de changement sont généralement utilisés indifféremment, ce qui n'est pas toujours judicieux, les réformes ne produisant pas toujours du changement et le changement n'étant pas toujours le produit d'efforts de réforme. La présente étude fait appel à la notion de réceptivité pour expliquer la pratique de la gestion du changement dans six pays de l'OCDE : la Finlande, la France, l'Italie, le Portugal, l'Espagne et la Suisse. Au cours des dernières années, ces six pays de I'OCDE ont adopté de grandes mesures de réforme pour moderniser la gestion de leur service public afin de répondre aux attentes croissantes de la société dans un contexte de ressources financières limitées et de pressions politiques. La réceptivité est un concept encore peu développé destiné à mettre en évidence les facteurs qui conduisent les organisations à se prêter peu, pas du tout ou très bien au changement. La présente étude soutient que la gestion du changement est une variable indépendante dans l'analyse du changement dans l'administration car elle détermine en grande partie la réussite de l'action publique. Quatre facteurs d'analyse étroitement liés sont utilisés pour expliquer la gestion du changement dans l'administration : vision idéologique, conduite du changement, politique institutionnelle, et capacité de mise en œuvre.

Le changement est axé sur la culture administrative, les valeurs traditionnelles, les priorités, les tâches quotidiennes, et par-dessus tout la mentalité du personnel des organismes publics étant particulièrement en question. L'ampleur du changement demeure cependant incertaine. Le présent document conclut que même si la notion de réceptivité est un moyen d'analyser et d'expliquer le changement dans l'administration, elle n'a pas suffisamment de valeur explicative pour permettre de déterminer si le changement s'est réellement produit. De plus, il soutient que les pays de l'OCDE sous-estiment l'importance de la gestion du changement lorsqu'ils conçoivent et mettent en œuvre des réformes de l'action publique. Cela tient au fait que les instruments utilisés pour gérer le changement correspondent plus à une méthode casuistique de formulation des politiques qu'à une stratégie délibérée de gestion des effets de la mise en œuvre d'une proposition de réforme. Aucun élément qui atteste de l'existence d'une stratégie cohérente de gestion du changement parallèle aux activités de réforme n'a été identifié. 


\section{Table des matières}

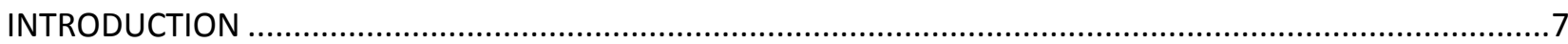

PARTIE I -- COMPRENDRE LE CHANGEMENT DANS L'ADMINISTRATION ......................................10

1. QU'EST-CE QUE LA GESTION DU CHANGEMENT DANS L'ADMINISTRATION ? ................................10

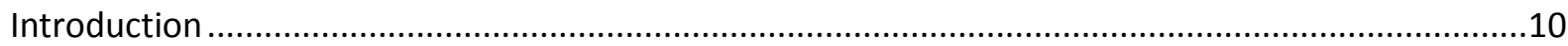

Les notions de changement et de réforme ......................................................................... 10

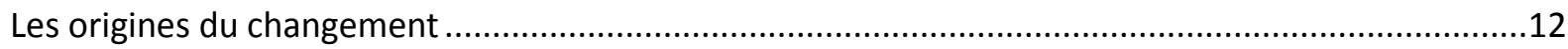

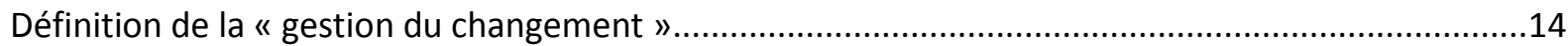

Réforme du service public et gestion du changement .........................................................16

2. EXPLIQUER LE CHANGEMENT DANS L'ADMINISTRATION - LA NOTION DE « RÉCEPTIVITÉ »..............19

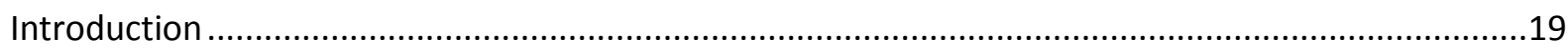

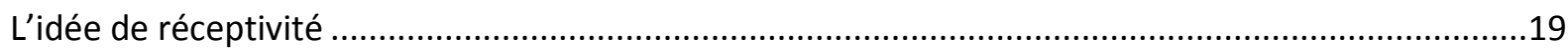

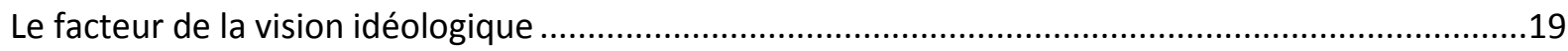

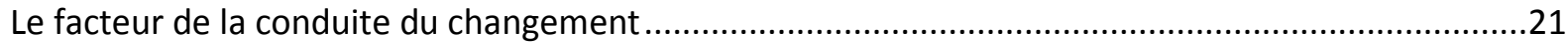

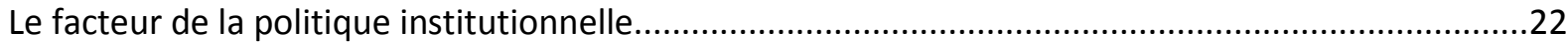

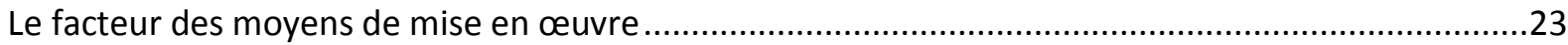

PARTIE II -- LA PRATIQUE DE LA GESTION DU CHANGEMENT DANS LES PAYS DE L'OCDE .....................26

3. LA MANIÈRE DONT LES PAYS DE L'OCDE GÈRENT LE CHANGEMENT - UNE COMPARAISON DES

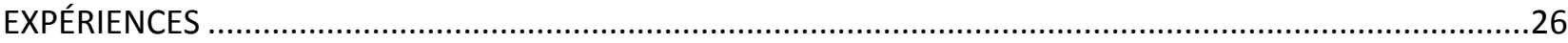

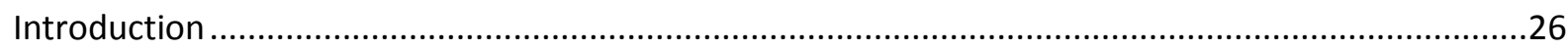

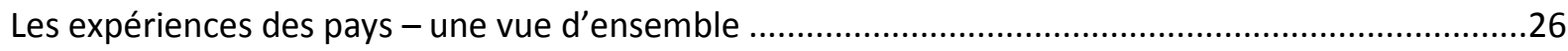

Facteurs à l'origine du besoin de changement.......................................................................28

Comparaison des expériences - Comment le changement est-il géré dans les pays de l'OCDE ?.......29

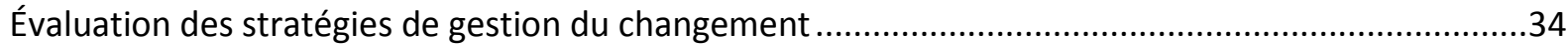

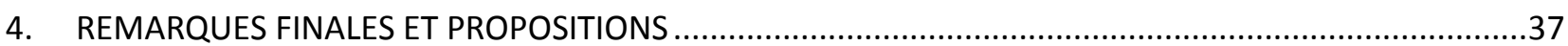

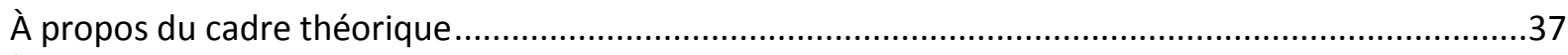

À propos de la pratique de la gestion du changement dans les pays de l'OCDE ...............................37

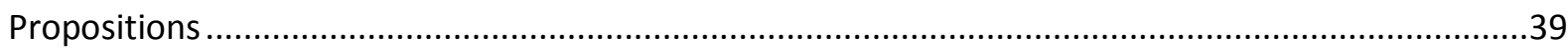

La route à suivre en matière de gestion du changement ........................................................42

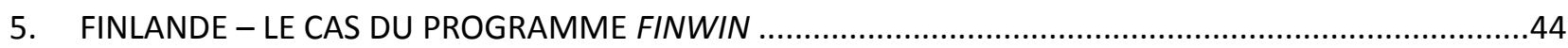

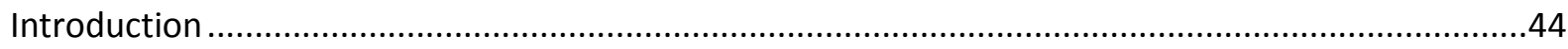

La force motrice -la population vieillissante de la Finlande ........................................................44

Affronter les difficultés liées à une fonction publique vieillissante - deux mesures de réforme ........45

Traiter le problème du manque de leadership - le programme Finwin ..........................................45

La perception du programme par le public ............................................................................46

En conclusion -- quels sont les enseignements pour la gestion du changement ? ..........................47 
6. LA FRANCE - LE CAS DE LA LOI ORGANIQUE RELATIVE AUX LOIS DE FINANCE...................................48

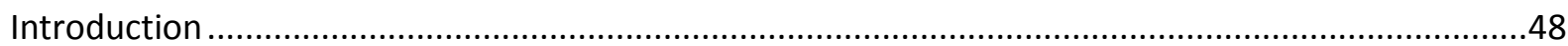

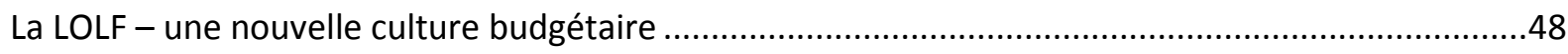

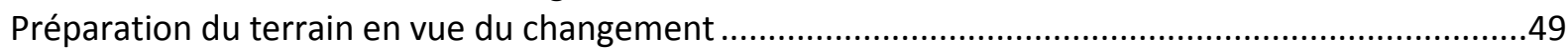

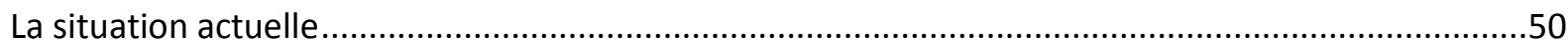

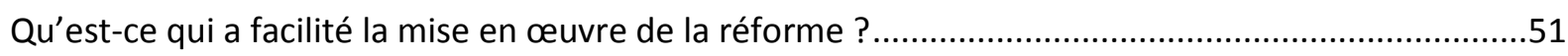

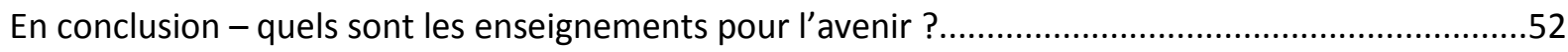

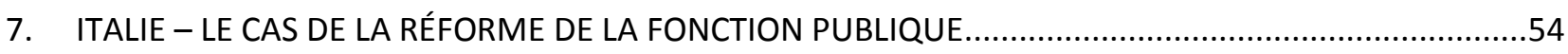

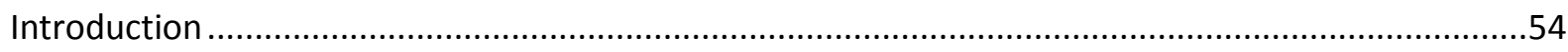

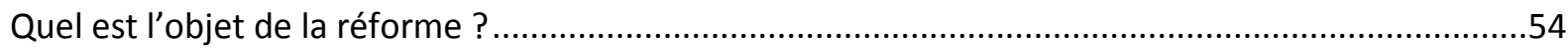

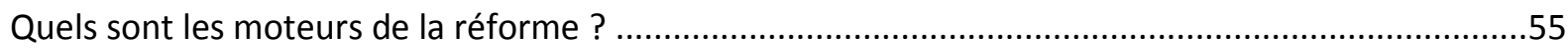

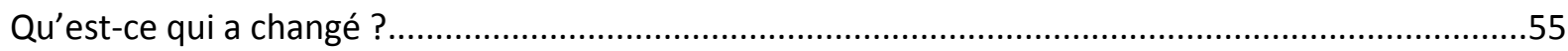

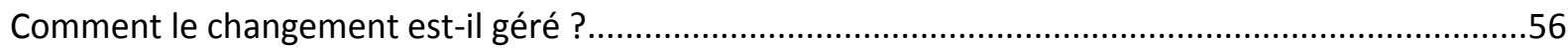

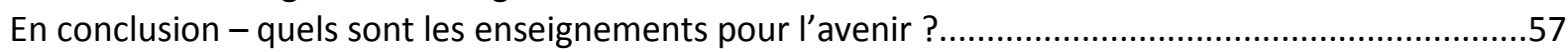

8. PORTUGAL - LE CAS DU PROCESSUS DE RÉFORME DE L'ADMINISTRATION PUBLIQUE .....................58

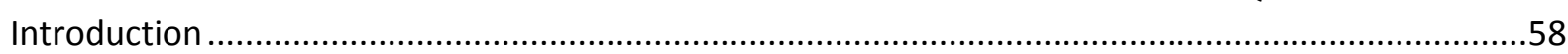

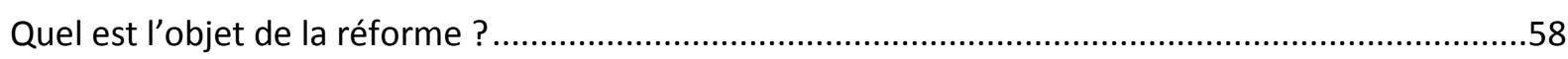

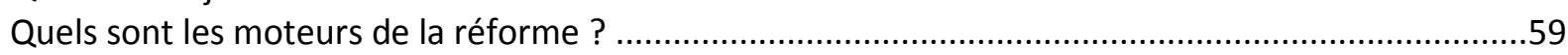

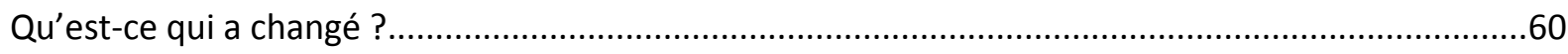

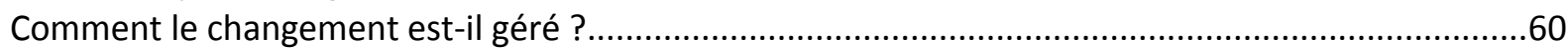

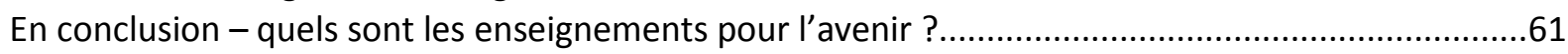

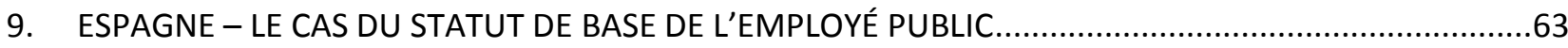

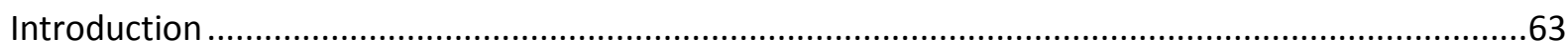

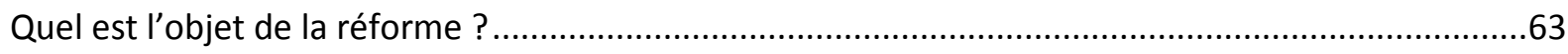

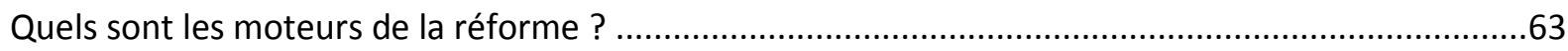

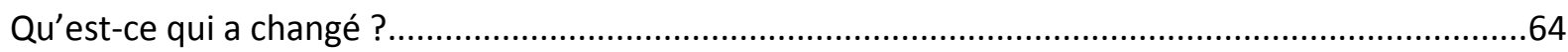

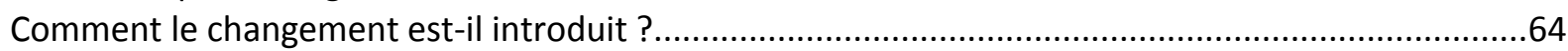

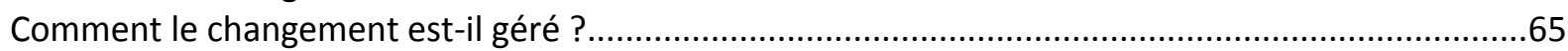

En conclusion - quels sont les enseignements pour l'avenir ? ........................................................66

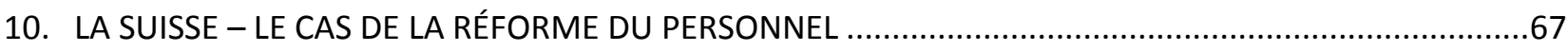

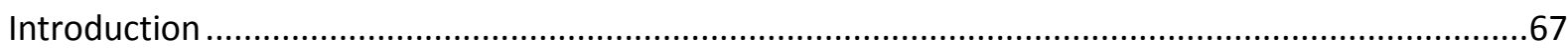

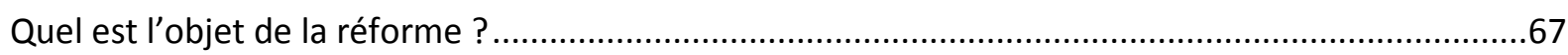

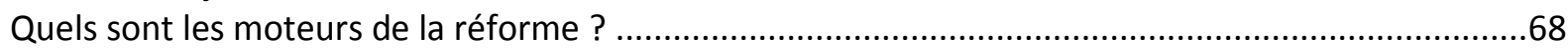

Qu'est-ce qui a changé ?

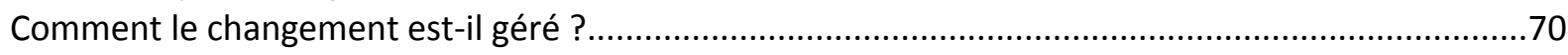

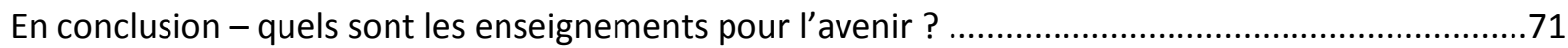

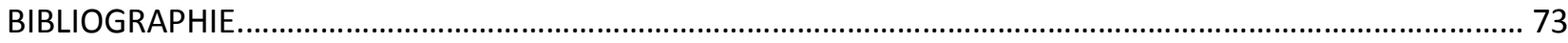




\section{INTRODUCTION}

1. Le monde actuel est très concurrentiel et exigeant. La société est mieux informée et attend plus des organisations publiques comme des organisations privées. Les processus et institutions publics traditionnels répondent moins bien aux besoins des individus. La mondialisation, I'utilisation généralisée des technologies de communication et d'information et l'émergence de la société du savoir, entre autres, sont en train de modifier rapidement l'ordre mondial. Cela a engendré de nouveaux défis pour les Étatsnations, les attentes des individus à l'égard des pouvoirs publics ayant augmenté, les demandeurs d'emploi étant plus exigeants quant au contenu des postes, et les sociétés préconisant davantage d'investissements dans l'enseignement, la santé et le collectif, mais étant peu désireuses de payer plus d'impôts (OCDE, 2005). Les systèmes de gestion du personnel s'adaptent moins bien à ces nouveaux défis. En effet, les pratiques traditionnelles de l'administration publique sont nées de contextes différents dans lesquelles les priorités étaient différentes. Désormais, les gouvernements jouent un nouveau rôle dans la société et assument de nouvelles responsabilités mais manquent généralement des outils nécessaires pour les gérer de façon constructive. On attend des gestionnaires publics qu'ils améliorent leurs performances en mettant l'accent sur l'efficience, l'efficacité et la bonne administration, qui ne figuraient pas parmi les priorités il y a 50 ans. De ce fait, pour être en mesure de s'adapter à un environnement en transition, le secteur public doit transformer ses structures, ses processus, ses procédures et surtout sa culture.

2. Dans ce nouvel ordre, la gestion du changement a été identifiée comme une variable déterminante pour la réussite ou l'échec d'une politique de réforme. La gestion du changement vise à s'assurer que les conditions nécessaires à la réussite d'une action de réforme sont réunies. Une politique de réforme peut faillir à apporter le changement, peut générer des résultats inattendus ou se heurter à la résistance d'organisations et/ou d'individus dont les intérêts sont touchés. C'est pour cela que les responsables de l'élaboration des politiques et les hommes politiques doivent accorder une attention particulière aux questions telles que le leadership, une vision commune, l'ordre de succession, les ressources nécessaires au changement et les valeurs culturelles, lorsqu'ils conçoivent et mettent en œuvre une action de réforme.

3. L'objet du présent document est de donner un aperçu général de la manière dont les pays de I'OCDE gèrent le changement dans le service public. Le présent document vise en particulier à : 1) identifier les variables déterminantes pour l'analyse de la gestion du changement dans le secteur public ; 2) confronter ces variables à l'expérience des pays Membres de I'OCDE pour déterminer leur pertinence ; et 3) tirer des enseignements de ces expériences en définissant de meilleures pratiques pour la gestion du changement dans le secteur public.

4. Les questions principales auxquelles le présent document s'efforce de répondre sont les suivantes: comment les gouvernements de l'OCDE gèrent-ils des changements aussi profonds, sur lesquels ils ont très peu ou pas du tout de contrôle ? Ces changements sont-ils concrètement intégrés dans la culture du service public ? Qui assure la gestion ? Enfin, les gouvernements disposent-ils au sein de leur personnel des compétences nécessaires pour conduire le service public vers le changement ? La réponse à ces questions s'inspire du débat et des conclusions de la réunion du Groupe de travail sur l'emploi et la gestion publics de l'OCDE des 28-29 novembre 2007. 
5. Le présent document fait appel à la notion de " réceptivité " pour expliquer la manière dont les pays de l'OCDE intègrent et gèrent le changement. Pour ce faire, il utilise quatre facteurs de réceptivité (Pettigrew, 1997; Butler, 2003) pour structurer l'analyse: la vision idéologique, la conduite du changement, la politique institutionnelle et la capacité de mise en œuvre. Le document relève que la réforme et le changement sont deux concepts distinctifs mais étroitement liés. La réforme est considérée comme le moyen pour les pouvoirs publics de produire du changement. C'est une activité délibérée destinée à modifier les processus, les procédures, les attitudes et les comportements. Le changement, quant à lui, est le fruit de la mise en œuvre de la réforme qui se traduit par un nouvel environnement administratif ou de nouvelles dispositions institutionnelles. La gestion du changement conditionne en grande partie la réussite ou l'échec de l'action publique parce que les réformes ne sont pas sans risque et peuvent avoir des conséquences inattendues ou être perçues de façon négative. Partant, ce document soutient que la gestion du changement constitue un moyen de réduire les niveaux de risque des réformes et qu'il faut par conséquent la considérer comme une variable indépendante dans l'analyse de la réussite de l'action publique. L'idée de réceptivité est indispensable pour expliquer et comprendre la gestion du changement, et des enseignements pourraient en être tirés pour des processus de réforme futurs. La notion de réceptivité est destinée à mettre en évidence les facteurs qui font que des organisations se prêtent peu, pas du tout ou très bien au changement.

6. L'idée de réceptivité permet de dégager des facteurs analytiques du processus de changement, elle facilite les comparaisons entre différents processus de changement, ce qui permet d'établir des généralités et des particularités, et elle intègre un large éventail de facteurs d'analyse abordés dans les travaux publiés sur la gestion du changement tels que le leadership, la communication, la vision et I'implication. Cependant, selon Pettigrew (1997), il n'existe aucune tradition théorique pertinente sur laquelle s'appuyer, la notion de réceptivité étant encore peu développée. De plus, ses arguments et conclusions doivent être étayés par des données empiriques détaillées, lesquelles ne sont pas toujours disponibles car il n'existe pas de phénomène de causalité en matière de production du changement. La notion de réceptivité s'appuie sur des données qualitatives, ce qui peut donner lieu à une certaine subjectivité et à des conclusions partielles en raison d'une d'observation limitée.

7. Les conclusions du présent document s'inspirent de l'analyse de six études de cas présentées lors de la réunion du Groupe de travail sur l'emploi et la gestion publics. Les critères de sélection des études de cas ont été les suivants : a) de grandes réformes ayant le potentiel de produire du changement dans la culture du secteur public ; b) des réformes qui couvrent différents domaines de la gouvernance ; c) des réformes mises en œuvre au cours des dix dernières années; et d) des mesures de réforme de pays disposés à présenter leurs expériences lors de la réunion du Groupe de travail sur l'emploi et la gestion publics.

8. Travailler avec ces études de cas présente un triple avantage. Premièrement, elles couvrent différents domaines de l'action publique (budgétisation, gestion des ressources humaines, évolution institutionnelle, etc.) qui illustrent la concrétisation de différents types de changement : opérationnel, stratégique, culturel et politique. Deuxièmement, elles se déroulent à différents niveaux de la gouvernance, ce qui enrichit le débat et renforce la pertinence des conclusions. Enfin, il s'agit de programmes de réforme continus, ce qui garantit l'actualisation des informations.

9. Ce document est divisé en trois parties principales. La partie I se propose de faire une synthèse des éléments théoriques permettant de comprendre et expliquer la gestion du changement dans le secteur public. Elle examine les concepts de réforme et de changement et explique en quoi la notion de réceptivité est utile pour comprendre le changement dans le secteur public. La partie II concerne la 
pratique de la gestion du changement dans les pays de l'OCDE. Elle procède à un vaste examen des constatations des études de cas et tire quelques conclusions générales. Enfin, la partie III décrit l'expérience de la Finlande, de la France, de l'Italie, du Portugal, de l'Espagne et de la Suisse en matière de gestion du changement dans le service public et s'en sert pour étayer les conclusions.

10. Le présent document a pour but de contribuer au débat sur la gestion du changement dans le secteur public en comblant les lacunes des travaux publiés sur la gestion publique. Son originalité et sa contribution résident dans I'utilisation de la notion de réceptivité pour expliquer des cas de gestion du changement dans une optique comparative. Les études de cas sont des travaux originaux, ces mesures de réforme n'ayant pas été analysées du point de vue de la gestion du changement. De plus, ce document est destiné à contribuer au développement de la notion de réceptivité comme cadre uniforme permettant d'expliquer et de comprendre le changement. Les conclusions du présent document montrent qu'il faudra des travaux de recherche et des débats supplémentaires pour mettre au point une méthodologie de gestion du changement propre à guider les travaux des décideurs et des responsables de l'élaboration des politiques. En ce sens, le présent document ouvre un nouveau champ pour la recherche dans I'OCDE, car il introduit des questions ou des perspectives intéressantes pour de futurs programmes de travail. 


\section{PARTIE I -- COMPRENDRE LE CHANGEMENT DANS L'ADMINISTRATION}

\section{QU'EST-CE QUE LA GESTION DU CHANGEMENT DANS L'ADMINISTRATION ?}

\section{Introduction}

11. L'introduction du changement dans le secteur public est très controversée et complexe car elle touche, directement ou indirectement, aux intérêts et aux modes de vie des membres de la société. Les organisations publiques et privées ne peuvent faire l'économie du changement si elles veulent survivre dans un environnement de plus en plus mondialisé, très interdépendant et en évolution rapide. La présente section a pour objet d'étudier les concepts de réforme et de changement, d'examiner les sources du changement, de définir ce qu'est la gestion du changement et de démontrer l'importance qu'elle revêt dans le contexte du service public.

\section{Les notions de changement et de réforme}

12. Le changement et la réforme sont deux concepts communément utilisés dans le discours politique et intellectuel. En politique, par exemple, l'élaboration des politiques est décrite comme une activité orientée vers le changement destinée à transformer, modifier ou revoir le statu quo. Les notions de changement et de réforme sont habituellement utilisées indifféremment, ce qui n'est cependant pas nécessairement toujours judicieux. La réforme n'est qu'une manière parmi d'autres de produire du changement; elle correspond à une méthode particulière de résolution des problèmes. Parfois la moindre réforme engendre des changements dans les organisations, dans d'autres cas certaines réformes ne débouchent sur aucun ou quasiment aucun changement. Si le changement sous la forme d'une transformation ou d'une modification peut être ou non un phénomène volontaire, la réforme est un processus structuré et délibéré de production du changement, quelle que soit son ampleur. Les réformes peuvent concerner les domaines politique, économique, social et administratif et intègrent des réflexions sur les problèmes et les solutions. Butler (2003), quant à lui, soutient que le changement est historique, contextuel et processuel.

Le changement est historique en raison d'un lien horizontal à travers le passé, le présent et le futur, il est contextuel en raison d'un lien vertical à travers différents niveaux de la société et il est processuel parce qu'il relie entre eux les processus et l'action. (Butler, 2003 : S50)

13. Deux aspects intéressants de cette conceptualisation du changement doivent être mis en évidence. Le premier est que le changement peut être présenté comme un processus qui décrit la manière dont les choses changent avec le temps. Pettigrew (1997 : 338) définit ce processus comme une suite d'événements, d'actions et d'activités individuels et collectifs qui se déploient 
progressivement en contexte. Le deuxième aspect est que le changement est dépendant du contexte. En ce sens, contexte et action sont liés.

Le contexte n'est pas seulement un environnement stimulant mais un système imbriqué de structures et de processus façonnés par les interprétations subjectives des acteurs qui perçoivent, appréhendent, apprennent et se rappellent. (Pettigrew, 1990 cité dans Butler, 2003 : S50)

14. Selon Brunsson (2006), l'existence de problèmes, un large éventail de solutions et l'oubli sont les éléments nécessaires à la concrétisation des réformes. La perception de problèmes présents et futurs d'une organisation, qu'elle soit publique ou privée, incite à mettre en oeuvre des changements. Par exemple, l'écart entre ce qu'une organisation est supposée fournir et la réalité peut déclencher le besoin de réforme. II arrive aussi que les réformes aient des conséquences inattendues qui justifient l'adoption de nouvelles mesures de réforme. Les réformes exigent également un bon stock de solutions potentielles aux problèmes présents ou futurs, lesquelles sont parfois fortement tributaires des modes intellectuelles et/ou pratiques en matière de résolution des problèmes. Brunsson (2006) souligne que la mode peut réduire ou accroître la diversité des solutions car elle limite le nombre de solutions acceptables. À la fin des années 80 et au début des années 90 par exemple, la Nouvelle gestion publique (NGP) était considérée comme le succès du moment dans les travaux publiés sur la gestion publique (Peters, 1997 ; Pollitt et Bouckaert, 2004) et elle a contribué à généraliser l'utilisation d'une méthode axée sur l'usager, la délégation et l'individualisation, supposée se prêter le mieux à la modernisation de la gestion du service public. À I'heure actuelle, le nouveau succès du moment est l'approche à l'échelle de l'administration (OCDE, 2005 ; Christiensen et Laegreid, 2007), qui propose une coordination de nature plus horizontale et non uniquement verticale afin de remédier aux inconvénients de la NGP. ${ }^{1}$ Les réformes peuvent aussi être la répétition d'anciennes réformes quelque peu remaniées. Lorsqu'une proposition de réforme perd de son efficacité et que la complexité s'accroît, d'anciennes solutions sont ressuscitées. ${ }^{2} C^{\prime}$ est pourquoi l'oubli facilite la réalisation des réformes.

15. Les réformes ne sont pas sans risque. Elles peuvent être soutenues ou repoussées par certains en fonction de leur point de vue, elles peuvent produire des résultats inattendus, elles peuvent être difficiles à mettre en œuvre, elles peuvent créer le besoin de nouvelles réformes, ou elles peuvent tout simplement ne pas fonctionner et mettre en danger la survie et la légitimité des organisations publiques et leur leadership managérial et politique. Partant, un aspect important des réformes est la gestion du changement ou des effets produits par sa mise en œuvre. Cette réalité suscite deux questions : comment gérer le changement produit par un processus de réforme, et quelles caractéristiques les réformes doivent-elles posséder pour être gérables? Le présent document portera particulièrement sur des cas de réforme administrative constituant une activité

1 L'exemple du Royaume-Uni montre comment les modes intellectuelles et pratiques influent sur l'élaboration des politiques ; l'administration Thatcher, par exemple, mettait fortement l'accent sur des éléments de NGP pour la réforme du service public tandis que les nouvelles politiques travaillistes sous l'administration Blair étaient axées sur l'approche à l'échelle de l'administration.

2 Cet aspect peut être illustré par les phénomènes de nationalisation et de privatisation. Au Mexique, par exemple, en 1982 les pouvoirs publics ont décidé de nationaliser toutes les banques privées pour gérer la crise économique engendrée par la corruption à l'intérieur des institutions et la chute des prix du pétrole. Dix ans plus tard, l'administration mexicaine a considéré que le meilleur moyen de renforcer le développement économique du pays était de privatiser les banques. 
ou un effort délibéré pour transformer le mode de fonctionnement ou de gestion des organisations publiques.

\section{Les origines du changement}

16. Le besoin de changement naît de l'insatisfaction suscitée par la situation du moment ou la perception d'un problème. Cette situation, ainsi que le laisse entendre Brunsson (2006), pousse à adopter une proposition de réforme qui envisage ou présente la vision d'une situation améliorée. L'insatisfaction peut avoir différentes sources telles que des réglementations, processus et procédures et/ou idéologies obsolètes. Stoker (2006) soutient par exemple que les citoyens de démocraties parvenues à maturité sont de plus en plus désabusés vis-à-vis du processus politique, ce dont témoigne une faible affiliation aux partis politiques, une baisse de la participation aux processus électoraux et un manque de confiance à l'égard des hommes politiques. Cette situation a amené les gouvernements à mettre en œuvre des réformes pour faire en sorte que le scénario politique soit plus ouvert.

17. De plus, les organisations publiques font partie d'un système de gouvernance à plusieurs niveaux (Evans, 2004). Le besoin de réforme est dû à des facteurs externes et/ou internes se situant à différents niveaux de la gouvernance. Les développements intervenant à certains niveaux de la gouvernance déclenchent le changement à d'autres niveaux. Le fait d'admettre l'importance et I'influence de ces éléments dans le processus de formulation des politiques permet de comprendre pourquoi et comment les changements se produisent.

\section{Sources externes}

18. L'environnement extérieur évolue rapidement et les pays ont de plus en plus besoin de s'adapter. Cependant, ils sont limités par l'interférence complexe d'agents des secteurs publics et privés aux niveaux mondial, international, national et local. Des interactions constantes et le fait de dépendre des ressources ont poussé les gouvernements nationaux à intégrer des réseaux d'action publique auto-organisés et interdépendants. ${ }^{3}$ Les pays ont ainsi vu leur capacité à définir leurs programmes de réforme de manière indépendante significativement restreinte, l'introduction de changements pouvant engendrer une réaction de la part d'autres membres du réseau du fait de leur interactions. En ne tenant pas compte des intérêts des autres agents, on peut provoquer l'échec de l'action et une résistance au changement.

19. Le transfert d'idées, d'innovations, et de meilleures pratiques entre pays a été facilité par des sources mondiales, internationales et transnationales de changement d'orientation. Le niveau de dépendance entre les membres d'un réseau d'action publique détermine le caractère coercitif ou volontaire d'un processus de transfert de politique et, ce faisant, il contribue à notre compréhension des origines du changement dans l'administration. ${ }^{4}$

\footnotetext{
${ }^{3}$ Pour un examen plus détaillé des réseaux d'action publique, de la gouvernance et du transfert de politiques, voir Rhodes (1999) et Evans (2004).

${ }^{4}$ Voir Evans (2004) pour un examen plus détaillé de formes «volontaires » et «coercitives » de transfert de politiques.
} 


\section{Encadré 1. Comprendre le « changement » - les sources externes}

- Une gestion efficace du changement exige la prise en compte des facteurs internes et externes qui ont déclenché le besoin de changement car ils déterminent l'attachement des individus à l'action de réforme (réforme du Trésor néo-zélandais).

- $\quad$ Des changements extérieurs au service public peuvent déclencher et définir des réformes administratives dans des organisations publiques (la crise économique de 1994-95 a conduit l'État mexicain à réformer la fonction publique puis à adopter des mesures pour améliorer la transparence dans le service public).

- Une interaction constante avec d'autres agents à différents niveaux de la gouvernance et le fait de dépendre de leurs ressources amènent les pouvoirs publics nationaux à intégrer un réseau d'action publique qui influe de manière formelle et/ou informelle sur les décisions politiques et sur le besoin de changement (ALENA, Mercosur et enceintes telles que le Groupe de travail international sur les biens publics mondiaux).

- $\quad$ Les décisions et priorités doivent être définies en respectant les intérêts des autres agents membres du réseau d'action publique à différents niveaux de la gouvernance afin de garantir des ressources, de limiter la résistance et de réduire le risque d'échec de l'action publique (ALENA, Mercosur et UE).

- $\quad$ Des forces mondiales, internationales et transnationales favorisent la concrétisation du changement dans le secteur public (politique environnementale grecque, programme mexicain de réforme administrative, réformes NGP en Asie du Sud-Est, réforme du Trésor néo-zélandais).

- $\quad$ Lorsque le besoin de changement est imposé par des forces extérieures (transfert coercitif), il est plus difficile pour l'ensemble des parties prenantes de s'impliquer dans l'action de réforme, ce qui peut compromettre la réussite de l'action de réforme (réforme du secteur de la santé au Kirghizstan).

\section{Sources internes}

20. L'insatisfaction des citoyens, une baisse de confiance à l'égard des pouvoirs publics et la participation du public à l'élaboration des politiques sont des forces qui déclenchent le changement dans l'administration. ${ }^{5}$ La capacité à gérer le changement efficacement dépend, dans une large mesure, de la participation ou non du public à la définition du programme de réforme. Bien qu'un processus d'élaboration des politiques ouvert exige davantage de consultation et de négociation, il incite de toute évidence à soutenir l'action de réforme, en particulier les solutions à long terme, donne de la légitimité au processus d'élaboration des politiques dans son ensemble, renforce l'implication de l'ensemble des parties prenantes à l'égard de la réforme, et facilite l'acceptation et la gestion du changement. ${ }^{6}$ Waugh $(2002$ : 379) par exemple soutient que la participation directe du public aux délibérations au sujet de l'action publique sur le risque environnemental fait qu'il y a plus

5 Pour les besoins du présent document, la participation publique est un concept générique qui fait référence à des groupes de la société civile tels que : les partis politiques, les ONG, les syndicats, les groupes savants, les membres de cercles financiers, les entrepreneurs et d'autres agents non étatiques. Pour un examen supplémentaire des avantages que présente la participation publique à l'élaboration de la politique, voir Waugh (2002). En ce qui concerne le renforcement de la confiance à l'égard des pouvoirs publics, voir OCDE (2006).

6 Il convient de noter qu'il est quasiment impossible de parvenir à des décisions à l'unanimité ; les politiques de réforme se heurteront toujours à une certaine opposition. Un consensus général peut permettre aux gouvernements de prendre une décision et d'entreprendre le processus de réforme. Si l'on attend l'unanimité, le processus risque d'être assez lent et le bon moment pour mettre en œuvre la réforme risque d'être dépassé. 
de chances que les orientations choisies correspondent aux besoins locaux et que les moyens locaux de réponse aux problèmes collectifs se développent.

21. Des événements internes tels que les crises économiques, l'agitation politique, une mauvaise gestion des catastrophes naturelles, les urgences sanitaires etc., peuvent également provoquer des changements à l'intérieur du secteur public dans le but de traiter plus efficacement les problèmes. Dans ce cas, la gestion du changement peut s'avérer un problème compliqué, le manque d'information et de sensibilisation, et même de préparation mentale et émotionnelle risquant de compromettre l'acceptation du changement. L'introduction d'une action de réforme pose le problème de l'adaptation à une nouvelle situation. Des réformes progressives, en revanche, faciliteraient la compréhension du changement et réduiraient la résistance.

\section{Encadré 2. Comprendre le « changement » - les sources internes}

- $\quad$ Les changements de gouvernement constituent un cadre propice au changement d'orientation (nouveau gouvernement travailliste britannique).

- Le manque de confiance à l'égard des pouvoirs publics peut favoriser un changement d'orientation (mesures en faveur de l'accès aux informations pour tenter de regagner la confiance des citoyens à l'égard des pouvoirs publics, réforme Copernic dans l'administration publique belge, Charte du citoyen au Royaume-Uni, réforme administrative mexicaine).

- $\quad$ Lorsque ce sont des forces ou groupes politiques, économiques et sociaux autochtones qui se rendent compte du besoin de changement, il est plus facile d'impliquer l'ensemble des parties prenantes dans la poursuite des résultats souhaités (flexicurité danoise, système néerlandais de retraite anticipée et d'allocations d'invalidité).

- La gestion du changement est facilitée par l'intégration des agents touchés par une action de réforme au processus de formulation des politiques (flexicurité danoise).

- Les réformes progressives facilitent la gestion et l'acceptation du changement et favorisent l'adaptation (réforme du Trésor néo-zélandais).

\section{Définition de la « gestion du changement »}

22. Comme indiqué plus haut, la réforme et le changement sont des phénomènes distincts mais étroitement liés. En ce sens, la gestion d'une réforme a plus à voir avec la mise en œuvre d'une proposition de réforme. Pressman et Wildavsky (1973 : xiii) définissent la mise en oeuvre à l'aide de synonymes tels que réaliser, mener à bonne fin, exécuter, produire, achever. Weissert et Goggin (2002 : 212), quant à eux, définissent la mise en oeuvre comme le processus de transformation de plans en actions, de traduction d'un projet de politique en un programme opérationnel réel. La gestion du changement désigne la manière de faire face aux conséquences volontaires ou non d'un programme de réforme. Selon cette logique, on peut avancer que la gestion du changement fait partie du processus de conception et de mise en œuvre d'une mesure des pouvoirs publics. L'objet du présent document n'est pas d'examiner la gestion des réformes, qui a été amplement analysée dans les travaux publiés sur la mise en œuvre; il mettra néanmoins l'accent sur la gestion du changement en tant que partie intégrante des processus de formulation et de mise en œuvre des politiques.

23. Même si la gestion du changement a été largement étudiée pour ce qui est de la gestion des entreprises (Garvin, 1993 ; Kotter, 1995 ; Butler, 2003 ; Nickols, 2006 en ligne ; Goman, 2000 en 
ligne ; Lorenzi et Riley, 2000 en ligne), les soins infirmiers (Baulcomb, 2003), l'aide sociale (McDonald et Harrison, 2004) et même la gestion de musées (Husion, 1999), elle n'a pas fait l'objet de la même attention dans les travaux publiés sur la gestion publique, ce qui est surprenant étant donné que les organisations privées comme les organisations publiques sont mises au défi de changer pour pouvoir survivre aux transitions qui touchent la société mondiale (Giddens, 2000 : 122). Dans le domaine privé, les clients exigent des services plus nombreux et de meilleure qualité, ce qui amène les organisations à changer rapidement pour répondre aux besoins de ces derniers et éviter d'être dépassées par leurs concurrents. Dans le secteur public, les citoyens attendent plus des pouvoirs publics, ce qui incite les organismes publics à modifier leurs structures et leurs procédures pour répondre efficacement à leurs demandes.

24. Selon Nickols (2006 en ligne), la gestion du changement signifie au moins deux choses. Tout d'abord, elle désigne la réalisation de changements d'une manière planifiée et systématique. Par exemple, les programmes de régionalisation et de productivité finlandais sont des mesures destinées à préparer le secteur public à affronter les difficultés liées à une population vieillissante. Ensuite elle désigne la réponse aux changements sur lesquels l'organisation a peu ou pas de contrôle, comme les crises économiques et les troubles politiques. Cependant, ces définitions gomment la différence entre réforme et changement. Si la réforme est une manière de faire face à un problème et que le changement est l'effet produit par la mise en œuvre de la réforme, alors ce que décrit Nickols ne correspond pas à des définitions de la gestion du changement mais à deux types de réforme : proactive et réactive.

25. Reed (1996) laisse entendre que la gestion de la culture et de la transformation culturelle est une compétence et une caractéristique de base de la gestion moderne. Cependant, Driscoll et Morris (2001) déclarent que les grands changements dans le secteur public sont problématiques ou ne sont viables que si les systèmes de valeurs et de croyances des membres de l'organisation connaissent un changement analogue. Partant, pour le présent document, la définition de la gestion du changement qui prévaut est celle de la capacité à influer sur les mentalités, la culture, les attitudes et les pratiques des gens pour les adapter à un nouvel environnement et à de nouvelles organisations. Pour commencer il faut convaincre les gens qu'il existe un problème et une solution plus viable, et par conséquent qu'une réforme est nécessaire. 


\section{Encadré 3. La gestion du changement dans le secteur public : les fondements}

- Le fait de comprendre pourquoi le changement se produit fournit les bases permettant de définir au mieux le mode de gestion du changement (réforme du Trésor néo-zélandais).

- $\quad$ La gestion du changement désigne l'adaptation des mentalités, de la culture et des attitudes des gens à un nouvel environnement, ce permet aux mesures de réforme de produire les résultats souhaités en surmontant les effets secondaires gênants et la résistance au changement (programmes de réforme administrative mexicains et portugais, nouvelles réformes de la gestion publique à Hong Kong, en Malaisie et à Singapour, flexicurité danoise, réforme de l'administration locale en Ukraine, New Deal britannique).

- La gestion du changement joue un rôle déterminant dans la réussite ou l'échec d'une action de réforme (flexicurité danoise, système néerlandais de retraite anticipée et d'allocations d'invalidité).

- Des processus de changement simultanés accentuent la complexité et le besoin de compréhension et de gestion du changement dans l'administration (processus de mondialisation et tradition).

- La réussite d'une réforme et la production du changement exigent le soutien et l'implication des hommes politiques, des fonctionnaires et de la société civile (flexicurité danoise).

- Les réformes ont leurs limites et comportent une grande part de risque car elles peuvent avoir des effets négatifs inattendus susceptibles d'exiger de nouvelles réformes (programmes de régionalisation et de productivité finlandais).

\section{Réforme du service public et gestion du changement}

26. Avec l'adoption d'idées et de techniques de NGP, les pays de l'OCDE ont connu un changement radical dans leur manière de fonctionner et de répondre aux exigences de la société par rapport à il y a 30 ans (Rhodes, 1999 ; OCDE, 2005; Dussauge, 2007). Peters (1997) a analysé l'ampleur de l'adoption des idées et techniques managériales par les pays de l'OCDE et conclu que même si le type de réforme était différent d'un pays à l'autre, certains éléments d'appréciation indiquaient une transition vers un secteur public à orientation plus managériale. La figure 1 illustre en quoi les valeurs traditionnelles de l'administration devraient être radicalement transformées par l'adoption d'un certain nombre de réformes de l'action publique. Bien que la portée de l'adoption de ces instruments de réforme soit variable, le but est très proche : l'instauration d'un service public fort et fiable, capable de répondre aux attentes des citoyens.

27. À l'heure actuelle les pays Membres de l'OCDE et les non membres sont en train de consolider les réformes s'inspirant de la $\mathrm{NGP}^{7}$ ou d'évoluer vers une nouvelle génération de réformes appelée l'approche à l'échelle de l'administration ${ }^{8}$, ce qui montre que le secteur public est en constante évolution. La difficulté actuelle des gouvernements est de gérer le changement produit par la mise en œuvre des programmes de réforme.

\footnotetext{
Pour un examen détaillé, voir OCDE (2007), Pollitt et Bouckaert (2004) et Rhodes (1999).

8 Voir Christensen et Laegreid (2007) pour un nouvel examen de l'approche à l'échelle de l'administration.
} 
Figure 1. Transformation culturelle dans l'administration

Valeurs traditionnelles

Hiérarchies de contrôle

Conformité

Caractère impersonnel du travail

Autorité liée au poste

Paradigme contrôle-commandement
Instruments de réforme

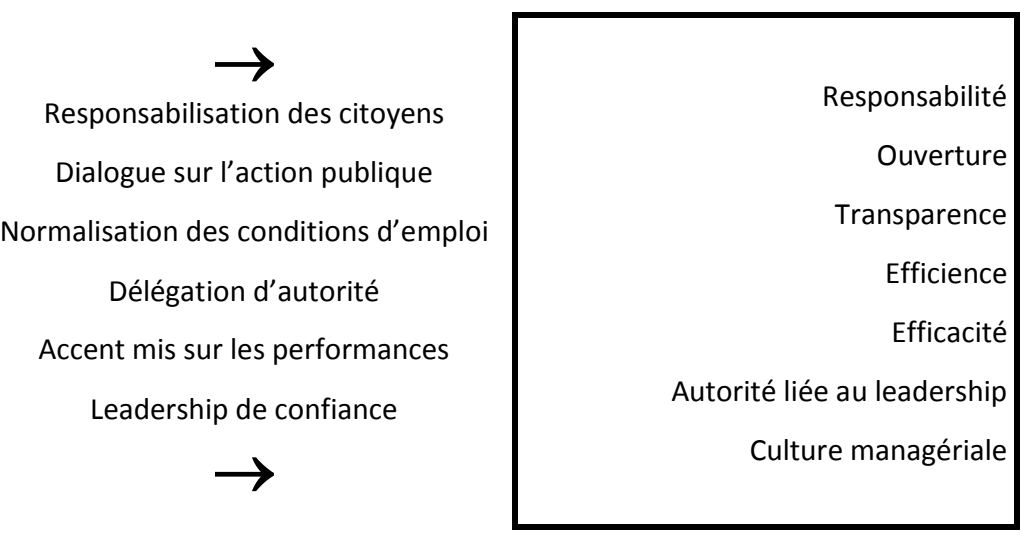

Source : OCDE (GOV)

28. Les réformes du secteur public sont complexes, dans bien des cas impopulaires, contestées, comportent une grande part de risque, et mettent beaucoup de temps à produire des résultats et à démontrer en quoi elles sont bénéfiques ${ }^{9}$, ce qui amène à s'interroger sur la manière d'entretenir la légitimité, d'accroître le soutien, de soutenir l'élan de réforme et d'éviter de continuer de perdre la confiance des gens tout en introduisant des mesures de réforme controversées mais nécessaires. Des techniques de gestion du changement efficaces devraient contribuer à maintenir l'élan de réforme tout en surmontant toute opposition au changement. L'encadré 4 recense certains des facteurs susceptibles d'être à l'origine d'une réaction négative au changement.

29. La complexité de la gestion du changement dans le secteur public est accentuée en cas de processus de changement simultanés qui font de la gestion et de la compréhension du changement une véritable gageure, et qui montrent à quel point il est nécessaire d'étudier la manière dont les pays gèrent en réalité les effets de leurs mesures de réforme. C'est pourquoi le présent document s'appuie sur deux hypothèses principales. Premièrement, il soutient qu'il faut considérer la gestion du changement comme une variable indépendante dans l'analyse de la réussite de l'action publique car elle détermine, dans une large mesure, la réalisation des objectifs de réforme. Deuxièmement, l'idée de réceptivité est essentielle pour expliquer et comprendre la gestion du changement dans le secteur public étant donné que l'adoption d'une proposition de réforme dépend de la réceptivité ou de l'ouverture au changement des organisations publiques. Cela tient au fait que la réceptivité des institutions et des fonctionnaires autochtones est un facteur déterminant du changement comme dans les cas de la politique de réforme environnementale grecque (Ladi, 2004), la réforme de l'administration locale en Ukraine (Ivanova et Evans, 2004), et la réforme de l'éducation en Équateur (Lana et Evans, 2004). La notion de réceptivité devrait fournir des éléments permettant d'esquisser quelques propositions pour l'élaboration d'une méthodologie de gestion du changement.

9 Pour un examen plus détaillé de la réforme des organismes de service public, voir Ferlie et al (2003). 


\section{Encadré 4. Facteurs entraînant une résistance au changement}

- Le manque d'homogénéité de la réforme et de compatibilité avec d'autres mesures de réforme peut entraîner de la confusion et susciter une opposition.

- La peur et l'incertitude face à un nouvel environnement de travail suscitent l'opposition à une action de réforme.

- Les implications négatives perçues par les individus et les groupes peuvent déclencher une résistance au changement.

- La complexité de l'action de réforme peut se traduire par une opposition si elle n'est pas clairement expliquée par les dirigeants et les gestionnaires et comprise par l'ensemble des parties prenantes.

- Le fait que le changement soit imposé peut susciter une opposition à l'action de réforme.

- Le changement peut être perçu différemment par les gens se situant à des niveaux différents de l'organisation.

- La réduction de l'importance du facteur humain dans le processus de changement.

- Le manque d'information et de communication peut accroître l'incertitude et le manque de confiance ce qui entraîne une résistance au changement. 


\section{EXPLIQUER LE CHANGEMENT DANS L'ADMINISTRATION - LA NOTION DE « RÉCEPTIVITÉ 》}

\section{Introduction}

30. Pour comprendre et expliquer le changement dans l'administration, il convient de répondre à certaines questions décisives : pourquoi le changement se produit-il et qu'est-ce qui le provoque, qui conduit le changement, que faut-il pour gérer le changement, et pourquoi les gens résistent-ils au changement ? L'objectif de la présente section est de définir les fondements théoriques de l'étude en s'appuyant sur l'idée de réceptivité. C'est pourquoi elle analysera ce qu'est la réceptivité. Ensuite elle expliquera chacun des quatre facteurs qui intègrent la notion de réceptivité : la vision idéologique, la conduite du changement, la politique institutionnelle et la capacité de mise en œuvre.

\section{L’idée de réceptivité}

31. Oliver et Pemberton (2004: 415) soutiennent que les moyens qui permettent de traduire les idées en action, et les conditions d'une évolution réussie des idées et de l'action publique demeurent incertains. Cependant, Butler (2003) laisse entendre que la notion de réceptivité est essentielle pour comprendre et gérer le changement dans les organisations du secteur public. La réceptivité est une notion en plein essor, mais encore peu développée, qui se propose de mettre en évidence les facteurs qui font que des organisations se prêtent peu, pas du tout ou très bien au changement (Butler, 2003 : S48). Bien qu'elle soit encore en cours de développement, l'idée de réceptivité peut fournir les outils analytiques permettant d'expliquer à quel point les organisations publiques sont ouvertes ou réceptives au changement et comment elles le gèrent. La réceptivité recouvre quatre facteurs d'analyse : vision idéologique, conduite du changement, politique institutionnelle, et capacité de mise en œuvre.

32. L'utilisation de la notion de réceptivité pour expliquer le changement et la manière dont il est géré présente trois avantages principaux. Premièrement, elle permet de dégager des aspects analytiques du processus de changement et de déterminer l'importance de chaque facteur dans la réalisation des objectifs de réforme et la production du changement. C'est un élément décisif car il n'existe pas de phénomène de causalité en matière de changement et les informations concernant les facteurs qui incitent les individus ou les organisations au changement sont limitées. Deuxièmement, elle facilite la comparaison entre différents processus de changement pour définir des tendances générales mais aussi pour déterminer ce qui fait le caractère unique de chacune des expériences de réforme. Et troisièmement, elle tient compte d'autres éléments d'analyse mentionnés dans les travaux publiés sur la gestion du changement tels qu'un leadership de confiance, des canaux de communication, une gestion des connaissances et de l'information performants, et l'implication des parties prenantes.

\section{Le facteur de la vision idéologique}

33. Butler (2003: S52) soutient que la vision idéologique fait référence à l'existence d'un programme stratégique, mais reconnaît que le programme peut résulter des intérêts d'un groupe défini au sein d'une organisation. Ce facteur comporte deux aspects que l'on retrouve souvent dans les 
travaux publiés : la vision et l'idéologie qui reflètent la culture du secteur public et sont les éléments qui cimentent l'organisation ou les organisations.

\section{Encadré 5. L'importance de l’homogénéité dans la gestion du changement}

- Les objectifs de la réforme doivent pouvoir être compris par l'ensemble des parties prenantes et compatibles avec d'autres mesures de réforme pour faciliter l'acceptation et la gestion du changement (réforme du Trésor néo-zélandais, flexicurité danoise).

- La compatibilité permet d'éviter confusion et effets secondaires négatifs, et favorise l'avènement du changement (réforme du Trésor néo-zélandais, mesures du Mexique en matière d'administration en ligne).

- Les réformes stimulent ou imposent généralement l'adoption de nouvelles réformes dans d'autres domaines et à d'autres niveaux de la gouvernance, et la compatibilité réduit le risque de situations confuses et accroît les chances d'acceptation des réformes (réforme du Trésor néo-zélandais, réforme de l'administration locale en Ukraine).

34. Selon Pettigrew (1997) la vision suppose une action publique de qualité et cohérente. En effet, la compatibilité avec des politiques de réforme plus générales est essentielle à la réussite d'une action de réforme. ${ }^{10}$ Elle doit correspondre aux moyens et à la nature du secteur public pour être intégrée au programme de réforme et pénétrer dans l'esprit des gens. Des réformes compatibles avec des politiques de réforme socio-économique générales sont considérées comme un élément favorable à la poursuite d'objectifs plus généraux qui, à leur tour, facilitent la gestion du changement. II est utile de souligner que les mesures de réforme dans un domaine ou à un niveau de la gouvernance stimulent ou provoquent généralement l'adoption de réformes dans d'autres domaines ou à d'autres niveaux de la gouvernance en raison de l'interdépendance des membres du réseau d'action publique. ${ }^{11}$ Par conséquent, la cohérence est essentielle si l'on veut éviter les réactions négatives des autres membres du réseau et préserver les ressources.

35. L'idéologie est un concept plus complexe car il désigne l'ensemble des croyances d'un groupe ou d'un individu particulier qui dictent son comportement. L'idéologie s'inspire des modes intellectuelles dans un contexte donné et est l'expression de l'aspect culturel du changement. La réceptivité des institutions et des fonctionnaires indigènes est un facteur déterminant du changement. Selon Driscoll et Morris (2001: 806), si le changement de comportement exige un changement dans la culture administrative, alors il se peut que le secteur public ait besoin d'une "révolution culturelle ». En effet, comme nous le verrons dans la deuxième partie du présent document, dans les pays Membres et non membres de l'OCDE la principale évolution culturelle a été l'effort pour rendre les organisations du secteur public plus managériales et moins bureaucratiques. La NGP, qui était supposée favoriser une culture administrative plus dynamique dans le secteur public, a donné la marche à suivre pour transformer le secteur public. Le gouvernement Thatcher en Grande-Bretagne et l'administration Reagan aux États-Unis peuvent être considérés comme les tournants dans cette transformation. Cependant, ces dernières années, les politiques s'inspirant de la NGP ont été considérées comme la raison de la fragmentation croissante du secteur public et des services publics. Cela tient au fait que la NGP a tendance à ignorer le problème de la coordination horizontale et que des outils tels que la

10 Voir Doig et Wilson (1998) et Wallis et Dollery (2001) pour avoir des exemples sur l'importance de la cohérence des politiques.

11 Wallis et Dollery (2001 : 204) ont constaté que pendant le processus de réforme du Trésor néo-zélandais, le personnel conseiller a eu toute latitude pour faire des propositions de réforme courageuses et novatrices à condition qu'elles tendent à faire progresser le processus parallèle de libéralisation, de stabilisation et de recentrage des pouvoirs publics sur leurs activités essentielles. Cette caractéristique a joué un rôle décisif dans la réussite de la réforme au sein de Trésor. 
gestion des performances et les organisations à fin unique ont tendance à mettre l'accent principalement sur la coordination verticale.

\section{Le facteur de la conduite du changement}

36. Le leadership est un élément déterminant dans la réussite de la mise en œuvre et la gestion du changement. La conduite du changement fait référence à l'idée que les dirigeants peuvent être des individus ou de petits groupes qui peuvent être issus de toutes sortes de métiers et de tout niveau hiérarchique d'une organisation (Pettigrew, 1997 ; Butler, 2003). Les dirigeants doivent pouvoir être en mesure d'inciter les gens à se concentrer sur une stratégie commune en donnant une autre signification à ce qu'ils valorisent, croient ou recherchent. Whiteley (1995) soutient que la séparation entre les "stratèges du changement" (hauts responsables), les "exécutants du changement " et les " bénéficiaires du changement " entretient le statu quo car les bénéficiaires n'entreprennent aucune activité de conception structurelle. Pour gérer efficacement le changement, il est nécessaire que les membres de l'organisation remplissent tous un peu des rôles de stratèges, d'exécutants et de bénéficiaires. Ce facteur d'analyse montre que tous des membres du personnel sont utiles à la production du changement.

37. Les dirigeants sont maintenant tenus d'obtenir l'implication et non plus la simple adhésion de leurs partisans. ${ }^{12}$ Pour faire face efficacement à un environnement changeant, les dirigeants doivent posséder les compétences nécessaires pour influencer leur peuple par la négociation, la persuasion et la persévérance. Les dirigeants ne sont pas nécessairement les auteurs ou les gestionnaires de la réforme, mais ceux qui peuvent stimuler et convaincre les gens et obtenir leur soutien. Si la gestion met l'accent sur des systèmes et processus formels, le leadership cherche en premier lieu à influencer les gens au moyen de valeurs et de visions d'avenir. Les dirigeants ne sont pas seulement des individus mais aussi des organisations. Les organisations publiques doivent conserver le premier rôle dans la formulation du programme de réforme de manière à ne pas connaître une perte d'autorité et pour être en mesure de gérer le changement plus efficacement. ${ }^{13}$

38. C'est aux dirigeants de faire en sorte que leur peuple accepte le changement. Lorenzi et Riley (2000 en ligne) soutiennent qu'un leadership efficace peut fortement limiter la résistance comportementale au changement. De la même manière, le document OCDE (2001) concluait que le leadership joue un rôle important dans la mise en oeuvre de la réforme du secteur public parce qu'il fait intervenir deux des aspects les plus importants de la réforme : le changement et les gens. Pour gérer le changement, les dirigeants ne doivent pas imposer les changements ou les réformes, mais privilégier l'adaptation. Pour ce faire, les dirigeants doivent être en mesure de persuader les gens de soutenir une cause ou une vision commune et en même temps de combattre la résistance au changement.

12 Pour un examen approfondi du leadership dans le secteur public, voir OCDE (2001).

13 Voir Wallis et Dollery (2001) pour un examen plus poussé du leadership au niveau d'une organisation s'appuyant sur le cas de la réforme du Trésor néo-zélandais et sur le changement de paradigme dans le secteur public. 


\section{Encadré 6. Le facteur de la conduite du changement dans la gestion du changement}

- Un leadership fort, de confiance et impliqué est un facteur déterminant dans la concrétisation d'une réforme (mesures de réforme du secteur public en Asie du Sud-Est, nouvelles politiques travaillistes en GrandeBretagne).

- Le leadership est un facteur déterminant pour réduire la résistance au changement (mesures de réforme du secteur public en Asie du Sud-Est, réforme du Trésor néo-zélandais).

- Un leadership efficace passe par l'obtention de l'engagement des gens par la persuasion, la négociation et en influençant leurs valeurs et leur culture (nouvelles politiques travaillistes en Grande-Bretagne).

- Même si les valeurs sont difficiles à changer, il est possible de donner une autre signification à ce qui est valorisé et, de cette manière, de transformer la culture de l'organisation (réforme du Trésor néo-zélandais).

- Des stratégies de perfectionnement du leadership sont indispensables pour préparer les dirigeants du secteur public à assumer leur rôle d'artisans de la réforme (I'ENA en France ; l'Office of Personnel Management aux États-Unis; le projet britannique sur le leadership de la haute fonction publique ; le conseil national suédois pour la qualité et le développement).

39. Le leadership fait partie intégrante des systèmes de gestion des ressources humaines. L'influence et l'importance du leadership varient d'un pays à l'autre mais son rôle dans la concrétisation du changement est communément accepté. Partant, la sélection des dirigeants et le développement de compétences de leadership sont indispensables à la gestion du changement. Les pays de l'OCDE tels que la France, l'Allemagne, le Mexique, la Nouvelle-Zélande, la Suède, le Royaume-Uni et les États-Unis ont adopté des stratégies de développement du leadership (OCDE, 2001). La formation des dirigeants dans le secteur public va de l'intervention directe de l'administration centrale comme dans le cas de I'École Nationale d'Administration (ENA) en France, à une approche de marché pour développer le leadership dans le service public, qui est adoptée dans des pays tels que la Nouvelle-Zélande.

\section{Le facteur de la politique institutionnelle}

40. La politique institutionnelle indique où sont prises les décisions dans les organisations publiques et privées. Butler (2003: S52) soutient que la politique institutionnelle témoigne de l'importance des réseaux coopératifs d'organisations. Ces réseaux sont avant tout inter-organisation, mais les membres du réseau peuvent également se situer à l'extérieur de l'organisation. Correctement gérés, les médias, par exemple, pourraient être des membres très utiles dans un réseau en préconisant le changement, une partie de leur travail consistant à définir les problèmes. La perception que les médias ont des politiques de réforme peut donner une idée de la façon dont la société réagira. C'est aux dirigeants de persuader les médias de soutenir une action de réforme.

41. Les réseaux d'organisations agissent comme des coalitions de cause et jouent un rôle fondamental dans l'introduction d'une réforme et la gestion du changement. Cela tient au fait que, comme le soutient Sabatier (1991:

Une coalition de cause se compose d'acteurs issus de nombreuses organisations publiques et privées à tous les niveaux de l'administration, qui partagent un ensemble de croyances (objectifs $d^{\prime}$ action publique et autres perceptions) et s'efforcent de manœuvrer les règles de différents organismes publics pour atteindre à terme ces objectifs.

42. Les coalitions de cause ont généralement de l'influence dans différentes sphères de l'action publique et à différents niveaux de la gouvernance, apportant un soutien économique et politique utile à une action de réforme à toutes les étapes du processus d'élaboration des politiques. Leur tâche est 
considérée comme terminée une fois le changement consolidé et les "nouvelles règles du jeu » intégrées à la culture des pouvoirs publics.

\section{Encadré 7. Le facteur de la politique institutionnelle dans la gestion du changement}

- La participation du public à la détermination du programme est indispensable pour obtenir la légitimité, le soutien et l'implication en faveur du changement (flexicurité danoise, politique mexicaine d'information du public).

- La négociation entre différents groupes au sein de l'organisation est nécessaire pour parvenir à un accord sur la nécessité d'entreprendre des programmes de réforme et obtenir et maintenir l'implication des parties prenantes à l'égard de la proposition de réforme (mesures de réforme du secteur public en Asie du Sud-Est, réforme du Trésor néo-zélandais).

- Les coalitions de cause dans les organisations publiques peuvent également accueillir des membres extérieurs issus des médias, des personnes influentes et des universitaires pour soutenir les changements dans le service public (processus de formulation de la politique mexicaine d'information du public).

\section{Le facteur des moyens de mise en œuvre}

43. La gestion du changement exige d'avoir les moyens d'action nécessaires. Butler (2003 : S52) laisse entendre que :

Les moyens de mise en œuvre désignent les mécanismes utilisés par ceux qui conduisent le changement pour façonner et influer sur la mise en œuvre de la stratégie/politique et le comportement d'autres parties prenantes dans le réseau institutionnel.

44. Le facteur des moyens de mise en œuvre fait ressortir l'importance de la disponibilité de compétences et de ressources (financières, matérielles, humaines, en connaissances et simultanément) au sein de l'organisation et de leur utilisation par les acteurs publics. Des moyens financiers et matériels ainsi que du personnel formé sont des éléments nécessaires à la réalisation des objectifs de l'action publique. Cependant, pour gérer le changement, les connaissances et le temps jouent un rôle encore plus important. Les connaissances dépendent de la disponibilité des informations qui permettent aux parties prenantes de comprendre la nécessité du changement, sa raison d'être et les moyens d'y parvenir. ${ }^{14}$ La disponibilité des informations favorise la mise en place de processus d'apprentissage à l'intérieur de l'organisation, ce qui crée les structures nécessaires au renforcement des capacités et à la production de connaissances locales. Les informations ne constituent pas des connaissances en soi. Ce sont l'analyse des informations et les enseignements qui en sont tirés qui engendrent les connaissances. Ce processus peut conduire les responsables de l'élaboration des politiques à produire de nouvelles solutions de réforme qui ont plus de chances d'être acceptées par les communautés d'action autochtones car considérées comme des innovations locales. En effet, Waugh (2002:380) soutient que les systèmes d'apprentissage favorisent l'adaptation à une situation changeante. Les collectivités ont plus de chances d'être innovantes et réceptives aux besoins locaux si elles disposent des informations techniques appropriées. Les dirigeants doivent par conséquent inciter les gens à créer et à partager des connaissances.

45. L'apprentissage est donc indispensable à la gestion du changement. Il existe, selon Oliver et Pemberton (2004), une relation entre l'apprentissage en matière d'action publique et le changement d'orientation. De la même manière, Garvin (1993) laisse entendre que pour accroître les chances de

14 Simon (1997) soutient que les institutions et les fonctionnaires vivent dans un monde de rationalité limitée qui fait que les informations ne sont pas toujours adaptées et disponibles au bon moment. 
réussite, les organisations doivent avoir l'apprentissage à cœur. Selon lui, un système d'apprentissage s'appuie sur cinq éléments constitutifs : i) résolution systématique des problèmes ; ii) expérimentation ; iii) enseignements tirés de sa propre expérience; iv) enseignements tirés de l'expérience des autres; et v) transfert de connaissances dans toute l'organisation. La création de connaissances peut permettre aux organisations publiques de répondre de manière plus performante et efficace aux attentes des gens. ${ }^{15}$ Cependant, les nouvelles idées doivent bénéficier d'un soutien politique pour être acceptées. En effet, selon Oliver et Pemberton (2004: 419) le triomphe des paradigmes de l'action publique dépend de l'aptitude des groupes d'intérêt à l'adopter, de leur capacité à promouvoir la nouvelle idée et à veiller à son adoption par ceux qui détiennent le pouvoir. Ainsi, il faut que les moyens de changer incluent le soutien politique, élément déterminant permettant de garantir l'adoption d'une action de réforme.

\section{Encadré 8. Le facteur des moyens de mise en oeuvre dans la gestion du changement}

- Une gestion efficace du changement exige que les organisations aient les moyens de changer (spécifiquement, l'accès aux ressources de tout type) et dépend de la capacité d'apprentissage de l'organisation et de son implication en la matière (réforme de l'éducation en Équateur, méthodologie Kaizen au Japon).

- La gestion du changement exige des ressources en temps et en connaissances plus que tout autre chose (réforme de l'éducation en Équateur, réforme de l'administration locale en Ukraine, réforme du Trésor néozélandais, réformes de la gestion publique au Mexique et en Asie du Sud-Est).

- Les connaissances et les informations créent un système d'apprentissage à l'intérieur de l'organisation qui favorise l'adaptation à une situation changeante (mesures du Mexique en matière d'administration en ligne).

- Le temps disponible pour le changement est généralement limité et la production du changement est très souvent un processus de longue haleine (réforme de l'administration locale en Ukraine, réforme du Trésor néo-zélandais, réformes de la gestion publique au Mexique et en Asie du Sud-Est).

46. Le temps est une ressource très utile qui n'est pas toujours disponible. La production du changement est un processus de longue haleine et les premiers résultats peuvent n'être visibles qu'après un délai encore plus long. En ce sens, Kotter (1995: 59) soutient que le processus de changement passe par une série de phases qui, au total, exigent généralement un laps de temps considérable. Une gestion efficace du changement doit veiller à maintenir l'élan de réforme pendant tout ce temps. Ainsi que Christensen et Laegreid (2007: 1063) le présentent, de nouvelles compétences, la modification de la culture institutionnelle, et l'établissement de relations de confiance mutuelle exigent de la patience. Le rôle d'un bon agent de la réforme est de se comporter plus en jardinier qu'en ingénieur ou en architecte. C'est également un travail psychologique car si les parties prenantes ne voient pas d'effets positifs à court terme, leur confiance à l'égard du leadership et leur intérêt pour la réforme elle-même risquent de s'émousser.

47. Par ailleurs, les objectifs de l'action publique sont très fortement dictés par des programmes politiques dont d'autres priorités peuvent se dégager en fonction du contexte politique du moment. La plupart du temps, le thème de la réforme administrative doit rivaliser avec des thèmes d'action publique qui peuvent être plus prioritaires pour les pouvoirs publics. Partant, les dirigeants doivent

15 L'apprentissage dans le secteur public a été amplement examiné dans les travaux publiés sur le transfert de l'action et les enseignements tirés des expériences; voir Evans (2004) et Rose (2005) pour une analyse approfondie. 
convaincre les hommes politiques des avantages de la réforme en dépit du fait que les résultats positifs ne se feront sentir qu'à plus long terme ${ }^{16}$.

16 Selon Wallis et Dollery (2001: 201), les décideurs qui sont réellement perplexes peuvent donc être disposés, à ce moment-là [moment de crise], à s'en remettre au leadership de réformateurs radicaux qui préconisent la reconstruction de l'action publique sur la base d'un nouveau paradigme, à condition que ce paradigme soit cohérent et qu'il fasse autorité. 


\section{PARTIE II -- LA PRATIQUE DE LA GESTION DU CHANGEMENT DANS LES PAYS DE L'OCDE}

\section{LA MANIÈRE DONT LES PAYS DE L'OCDE GÈRENT LE CHANGEMENT - UNE COMPARAISON DES EXPÉRIENCES}

\section{Introduction}

48. Les pays de I'OCDE sont confrontés à un nombre croissant de difficultés découlant de la mondialisation, de la délégation et de la transformation de la gouvernance. Les sociétés attendent plus des pouvoirs publics dans les domaines, entre autres, de l'éducation, de la santé, de la sécurité et de la protection de l'environnement. Le rôle des pouvoirs publics a considérablement changé avec leur intégration progressive à des réseaux d'action publique dans lesquels ils ne sont qu'un acteur parmi les autres dans le processus de prise de décision.

49. La réforme du service public a pour but d'améliorer la gestion des ressources humaines, de faire un meilleur usage des fonds publics, de fournir des services publics améliorés et de modifier l'image du service public en tant qu'employeur. Des contraintes économiques, des exigences sociales croissantes et une dépendance internationale de plus en plus grande font que des réformes sont indispensables si les pouvoirs publics veulent pouvoir répondre de façon constructive à ces défis.

50. L'objet de la présente section est d'analyser l'expérience de six pays de l'OCDE -- Finlande, France, Italie, Portugal, Espagne et Suisse -- en matière de gestion du changement produit par la mise en œuvre de réformes dans le service public. Pour ce faire, cette section présentera une vue d'ensemble des mesures de réforme. Elle mettra en évidence certains des facteurs qui ont conduit à réformer le service public dans ces pays. Elle analysera ensuite la manière dont ces pays de l'OCDE gèrent concrètement le changement en s'appuyant sur la notion de réceptivité. Enfin, elle mettra en évidence, en guise de conclusion, certains enseignements tirés de la pratique de la gestion du changement et elle esquissera un certain nombre d'éléments de base pour la mise au point d'une méthodologie de la gestion du changement.

\section{Les expériences des pays - une vue d'ensemble}

51. La Finlande, la France, l'Italie, le Portugal, l'Espagne et la Suisse ont assisté à la transformation progressive de leur culture administrative, leurs processus, leurs procédures et leur organisation institutionnelle étant incompatibles avec les besoins et priorités nationaux. Leurs systèmes de gestion du personnel traditionnels se sont avérés peu modulables pour répondre à de nouveaux défis nationaux et internationaux. Cependant, l'introduction du changement dans le service public constitue 
en soi un défi, les mesures de réforme se heurtant généralement à l'opposition de fonctionnaires et autres membres de la société qui continuent de douter de leur efficacité et de leurs bénéfices. ${ }^{17}$

52. Les finalités et les objectifs des mesures de réforme dans ces six pays de l'OCDE dépendent en grande partie sur leur contexte politique, historique, social et économique propre. Cependant, il est utile de noter qu'en dépit de situations différentes, l'objectif des programmes de réforme est, en général, d'améliorer l'efficacité du service public pour répondre aux attentes croissantes de la société dans un contexte de ressources financières limitées et de pressions politiques.

53. Le programme finlandais Finwin est destiné à remédier au manque de leadership et de communication dans le service public. La réussite du programme dépend en quasi-totalité de son impact positif sur les mesures de changement telles que les programmes de régionalisation et de productivité. L'expérience finlandaise sur le plan de l'introduction du changement dans le service public illustre les dangers d'un manque de stratégie de communication et de leadership impliqué. La formulation des politiques et la conception d'une stratégie de communication sont deux processus dépendants nécessaires à la concrétisation du changement. Cependant, ils doivent s'appuyer sur un dialogue entre l'ensemble des parties prenantes et un leadership impliqué.

54. Depuis peu la France applique pleinement la Loi organique relative aux lois de finances (LOLF) qui établit une nouvelle logique budgétaire. La LOLF vise à transformer une culture centrée sur les procédures en une culture orientée vers les résultats. La mise en oeuvre progressive de la LOLF a favorisé le développement d'un processus d'adaptation culturelle avant sa pleine mise en œuvre. Le soutien politique, un leadership impliqué, un processus de mise en œuvre progressive, une stratégie de communication, la formation et les tests sont les facteurs qui ont permis aux autorités françaises de gérer le changement culturel provoqué par la mise en œuvre de la LOLF.

55. La réforme de la fonction publique italienne montre que la production du changement est un processus de longue haleine qui exige, entre autres, une implication politique, la capacité de tirer des enseignements d'autres expériences, et une approche auto-critique. La mise en oeuvre de réformes d'inspiration managériale est censée changer la culture du service public. À l'heure actuelle, il s'agit pour la fonction publique italienne de consolider les réformes entreprises il y a plus de dix ans. Pour ce faire, les autorités italiennes adoptent une nouvelle méthode auto-critique pour évaluer leurs atouts et leurs faiblesses et tirer des enseignements des autres pays afin d'améliorer leur propre processus de réforme.

56. À l'heure actuelle le Portugal est en train de remanier sa stratégie de modernisation de l'administration. Cinq programmes de réforme ont pour but de faciliter l'introduction d'une culture de la gestion des performances mais leur mise en œuvre a été progressive, seuls deux d'entre eux étant opérationnels. Pour que ces mesures de réforme portent leurs fruits, il faut parvenir à faire évoluer les mentalités. Même si l'opinion des fonctionnaires sur la réforme n'est pas complètement favorable, les médias et les acteurs économiques jugent les mesures essentielles pour parvenir à la croissance économique. L'expérience portugaise de la gestion du changement dans le secteur public illustre combien il est opportun de mettre en oeuvre progressivement une réforme et la nécessité de maintenir la dynamique pour parvenir aux résultats souhaités.

17 Teresa Ganhão soutient que la résistance des fonctionnaires est naturelle dans tous les processus de changement [en particulier] lorsque la réforme de domaines sensibles liés à leurs conditions de travail est en jeu. [Teresa Ganhão, Département des relations internationales, de la communication et de la documentation du Portugal, dans ses commentaires sur l'étude portugaise]. 
57. L'Espagne a récemment mis en œuvre un cadre réglementaire général pour les employés publics à tous les niveaux de l'administration. Le statut de base de l'employé public (EBEP) est censé donner de la cohérence à des pratiques variées de gestion des ressources humaines dans le service public. Ce statut constitue une rupture importante dans la culture administrative traditionnelle espagnole car il introduit des pratiques à orientation managériale dans le secteur public. L'expérience espagnole résume à elle seule l'utilité d'instaurer le dialogue et la consultation avec l'ensemble des acteurs concernés dans le processus de formulation des politiques pour gérer le changement plus efficacement. La consultation et le dialogue permettent de mettre au point une vision commune à laquelle adhèrent la majorité des forces politiques et sociales.

58. Les pressions financières, des procédures rigides et coûteuses, et une compétitivité en baisse sur le marché du travail ont conduit les autorités suisses à réformer, entre autres domaines, son système de gestion des ressources humaines. Deux projets transversaux visent à optimiser la gestion du personnel et à simplifier le cadre juridique, et sont censés changer l'image du service public d'ici 2015. L'expérience de la Suisse témoigne du besoin d'élaborer une vision d'avenir pour donner une orientation aux efforts de réforme, et de l'utilité de mettre en place une coalition directrice pour diriger le projet de réforme.

\section{Facteurs à l'origine du besoin de changement}

59. Les éléments d'appréciation disponibles laissent supposer que les moteurs de la réforme dans les pays de I'OCDE sont principalement des facteurs structurels internes. Ils varient en fonction du contexte politique, économique et social local de chaque nation. Cependant, les études de cas analysées révèlent que les pressions financières constituent le principal moteur de la réforme (voir en particulier l'expérience de la France, de l'Italie, du Portugal et de la Suisse). Des ressources financières limitées conduisent les nations à rechercher de meilleures solutions pour faire davantage avec moins de moyens. La masse salariale représentant un pourcentage considérable du budget d'un pays, les gouvernements doivent réorganiser la fonction publique pour mieux utiliser les ressources financières et humaines. Les pressions politiques peuvent être considérées comme le deuxième moteur le plus important de la réforme. En effet, une répartition inégale du pouvoir comme dans le cas de la France, des pressions de la part de groupes politiques comme en Espagne, ou des acteurs économiques et des médias comme au Portugal, ont déclenché ou renforcé le besoin et le caractère urgent des réformes.

60. Des processus et procédures administratifs et budgétaires dépassés constituent une autre source de changement. II s'agit d'un moteur plus technique de la réforme qui a une incidence sur les sources politiques et économiques de changement. Les possibilités d'une société mieux informée, la nécessité d'une réponse plus efficiente et efficace aux attentes des citoyens et de mieux utiliser les ressources ont conduit les gouvernements à modifier leur mode de fonctionnement traditionnel. En Espagne, par exemple, il a fallu instaurer un cadre réglementaire général pour donner de la cohérence aux pratiques différentes de GRH des trois niveaux de l'administration. En France, il a fallu mettre en place un cadre budgétaire moderne pour améliorer la souplesse, la transparence et l'obligation de rendre des comptes dans la gestion de ses ressources financières. Les autorités italiennes ont reconnu leur efficacité limitée dans la gestion des fonds publics.

61. Les cas italien et finlandais présentent deux moteurs particuliers du changement. D'une part, l'Italie a été influencée par des sources extérieures de changement notamment en exploitant les enseignements tirés de l'expérience des autres nations de l'Union européenne (UE). Les expériences de réforme dans les pays de I'UE constituent une référence qui permet aux autorités italiennes de déterminer où elles se situent par rapport à leurs homologues européens. D'autre part, la Finlande tire des enseignements de son expérience dans la mise en œuvre de mesures de réforme (programmes de 
productivité et de régionalisation) pour élaborer un programme destiné à remédier au manque de leadership et de communication dans le secteur public et, ainsi, favoriser l'avènement du changement.

Tableau 1. Liens de causalité dans la réforme administrative

\begin{tabular}{|c|c|}
\hline Causes du changement & Pays \\
\hline Pressions financières & France, Italie, Portugal, Suisse \\
\hline Pressions politiques & France, Portugal, Espagne \\
\hline Processus et procédures dépassés & France, Espagne \\
\hline Influence externe & Italie \\
\hline Expérience locale & Finlande \\
\hline
\end{tabular}

Source : OCDE (GOV)

62. Le tableau 1 laisse à penser que la mise en œuvre de réformes administratives, en particulier en matière de $\mathrm{RH}, \mathrm{n}$ 'est pas en soi la principale préoccupation des gouvernements : la réforme du service public est un moyen de répondre à des exigences financières et politiques. En d'autres termes, l'importance des réformes de la GRH, par exemple, tient au fait qu'elles facilitent ou encouragent la réalisation d'autres objectifs de réforme dans les domaines économique et politique. On peut alors se demander si, en l'absence de pressions économiques et politiques, la réforme du service public en général, et de la GRH en particulier, aurait lieu. Les éléments d'appréciation disponibles semblent laisser supposer qu'en l'absence de facteurs de cohésion tels que ceux recensés au le tableau 1, la réforme du service public a peu de chances de se réaliser. En effet, les six expériences de réforme semblent indiquer que le meilleur moment pour entreprendre un processus de réforme est une période de contraintes économiques, d'agitation politique et/ou de mécontentement social. La nécessité d'un grand changement dans les six pays analysés apparaissait clairement du fait de résultats médiocres de l'administration en matière de gestion des ressources publiques et d'une réponse inefficace aux attentes des citoyens. En effet, le " paradoxe du changement " est le fait qu'un contexte de ressources limitées et de résultats médiocres permet de convaincre plus facilement les gens du besoin de changement mais laisse moins de marge de manœuvre, les ressources disponibles n'étant pas suffisantes. Dans une période de développement économique et de stabilité sociale et politique, il est plus difficile de justifier le besoin de changement malgré le fait que l'on dispose de ressources suffisantes pour y travailler. Par conséquent, pour entreprendre un processus de réforme, il semble essentiel d'établir le besoin et l'urgence du changement pour garantir l'adhésion au processus de réforme.

\section{Comparaison des expériences - Comment le changement est-il géré dans les pays de I'OCDE ?}

63. L'ampleur et le calendrier des expériences des six pays de l'OCDE présentées dans le présent document est variable, et elles couvrent différents domaines de réforme administrative: GRH, questions budgétaires, et leadership. Cependant, à l'aide de la notion de réceptivité, il est possible de distinguer les points communs et les divergences dans la pratique de la gestion du changement des pays de l'OCDE.

64. Vision idéologique. Comme nous I'avons indiqué plus haut, la vision idéologique fait référence à l'existence d'un programme stratégique, défini selon les intérêts d'un groupe spécifique de l'organisation, qui expose les solutions à un problème supposé ainsi que la culture de l'organisation. En effet, les études de cas révèlent que la création d'une vision commune est de la plus haute importance pour orienter les efforts de réforme. Cela a été le cas en Suisse, en France et en Finlande où les réformes s'appuient sur une vision d'avenir préférable et souhaitée. Cette vision doit être commune à tous les membres d'une coalition directrice ou à tous les dirigeants qui doivent la faire connaître à 
l'ensemble des parties prenantes et des acteurs concernés par les réformes proposées. Faute de vision, les efforts de réforme se transforment en projets ambigus et incompatibles. Selon Liuksia, une vision commune nous dit où nous allons, ce que nous voulons, et où nous serons dans dix ans ${ }^{18}$. La LOLF française s'appuie également sur une conception de l'avenir de la gestion des finances publiques plus transparente et souple. Les initiateurs de la LOLF sont considérés comme des visionnaires qui ont été en mesure de mettre en évidence le besoin de changement et d'expliquer les risques liés au maintien du statu quo. Les autorités suisses ont établi une vision de leur service public d'ici 2015 et désormais tout effort de réforme doit être orienté en ce sens. La Finlande est en train de mettre au point une vision du service public par le biais des discussions qui ont lieu pendant les séminaires organisés dans le cadre du programme Finwin. Le dialogue horizontal et vertical entre les gestionnaires permet aux autorités finlandaises de dresser un tableau général de l'avenir du service public ${ }^{19}$. L'expérience de la Finlande, de la France, du Portugal et de la Suisse illustrent la nécessité de faire connaître la stratégie et de partager des informations avec le plus grand nombre possible d'acteurs concernés ou touchés par le processus de transformation. La Finlande, par exemple, a rencontré des difficultés lors de la mise en œuvre de ses programmes de régionalisation et de productivité parce qu'elle n'avait pas suffisamment fait connaître les objectifs du programme.

\section{Encadré 9. La modernisation du service public irlandais}

Depuis 2002, I'Irlande met en œuvre un programme de modernisation approfondie du service public dans le cadre d'une approche de partenariat social. Les objectifs de la réforme sont a) de soutenir les pouvoirs publics en matière de développement national, b) de faire un usage plus efficace des ressources, et c) d'améliorer la qualité des services publics. Le dessein de la réforme est de convertir le service public en une organisation très performantes, ouverte et souple affichant les plus hauts niveaux d'intégrité, d'équité et de responsabilité. La nouvelle approche implique la participation de plusieurs acteurs à l'élaboration des politiques : administration plus entreprises/employeurs, syndicats, monde agricole, secteurs social et associatif. Le véritable changement devrait se faire sentir dans la qualité des services aux usagers.

65. La France, I'Italie, le Portugal, l'Espagne et la Suisse s'acheminent vers la mise en place ou l'introduction dans le service public de pratiques managériales qui sont supposées améliorer la prestation de services et favoriser un meilleur usage des ressources humaines et financières. Ces réformes reflètent, en règle générale, l'idéologie à la mode dans les cercles politiques, économiques et universitaires, à savoir la méthode managériale de réforme du service public. Cela peut s'expliquer par le fait que pour répondre aux pressions financières et politiques, les élites politiques et économiques préfèrent une réforme de type technique car elle ne remet pas en cause leurs intérêts. Il est difficile de s'opposer à une réforme dont le but est d'améliorer le service public pour répondre plus utilement et efficacement aux besoins des citoyens et, indirectement, améliorer l'utilisation des ressources financières. C'est peut être la raison pour laquelle elle a été communément acceptée dans les pays de I'OCDE comme une manière viable de réformer le secteur public (Peters, 1997). Cependant, il n'est pas possible de dire si la culture managériale est un bon moyen pour produire du changement dans le service public. II y a deux principales raisons à cela. D'une part, la culture managériale, comme nous l'avons vu plus haut, a été à l'origine d'une fragmentation et d'un manque de coordination horizontale dans les organisations publiques, en ignorant le fait que les réformes doivent être conçues dans le but

18 Présentation de Veikko Liuksia, du ministère finlandais des Finances, lors de la réunion du Groupe de travail sur l'emploi et la gestion publics, au siège de l'OCDE, les 28 et 29 novembre 2007.

19 Le programme Finwin illustre également la définition du programme de réforme dans le cadre d'une approche à l'échelle de l'administration, les gestionnaires de différents ministères et niveaux de la hiérarchie étant supposés définir les priorités de changement dans le service public. 
de changer l'attitude de différents acteurs. Les changements apportés à un élément du système influent ensuite sur d'autres éléments du système (OCDE, 2005). D'autre part, les études de cas analysées concernent des réformes en cours qui ne sont qu'au début de leur processus de mise en œuvre et dont les bénéfices ou les limites ne seront visibles que sur le long terme. Par exemple, la LOLF française elle-même a pour objet la transition d'une culture de moyens à une culture de résultats mais il faudra du temps pour que ce changement soit institutionnalisé.

66. La conduite du changement. Ce facteur d'analyse indique où sont prises les décisions et analyse les actions des dirigeants. Les éléments d'appréciation présentés dans les études de cas montrent que les décisions sont prises dans les cercles politiques et ceux des hauts responsables. Les processus de consultation, comme dans le cas de l'Espagne, ont pour but de justifier ou de donner une légitimité à une décision déjà prise. Bien entendu, les réformes peuvent subir quelques modifications au niveau de leurs aspects techniques mais l'identification du problème et du besoin de réforme, la manière d'envisager la solution, et le moment choisi pour la mettre en œuvre sont des activités menées par les membres des élites politiques et d'encadrement comme l'illustre le tableau 2. Le rôle de chef de file du ministère des Finances dans les réformes analysées renforce le sentiment que les réformes administratives sont l'instrument d'objectifs d'action plus vastes. Les questions de GRH sont liées à des objectifs financiers et économiques, d'où leur rôle majeur dans le processus de réforme. Dans une certaine mesure, cela contribue à maintenir l'élan de réforme dans l'administration publique. Cependant, le risque alors est que la réforme administrative soit définie plus en fonction des priorités économiques qu'en tenant compte de ce dont l'administration a véritablement besoin pour être plus rationnelle et efficace.

67. De plus, le point commun des six expériences des pays présentées est qu'elles donnent au secteur public un rôle déterminant dans la définition des stratégies permettant d'affronter les défis futurs tels qu'une population vieillissante, la recherche de l'équité, et le développement économique. En effet, une étude de l'OCDE a conclu qu'après 20 ans de réforme les attentes de la société vis-à-vis de l'État ont augmenté, et le rôle de l'État est plus important et de nature différente (OCDE, 2005).

Tableau 2. Conduite du changement dans les pays de l'OCDE

\begin{tabular}{|l|l|l|}
\hline \multicolumn{1}{|c|}{ Pays de l'OCDE } & \multicolumn{1}{|c|}{ Lieu de prise de décision } & \multicolumn{1}{c|}{ Qui met en œuvre la réforme ? } \\
\hline Finlande & $\begin{array}{l}\text { Élite d'encadrement (commission } \\
\text { ministérielle chargée de la politique } \\
\text { économique) }\end{array}$ & Ministère des finances \\
\hline France & $\begin{array}{l}\text { Élite politique (membres de l'Assemblée } \\
\text { nationale) }\end{array}$ & Ministère de l'économie et des finances \\
\hline Italie & $\begin{array}{l}\text { Élites d'encadrement (agents publics } \\
\text { élus) }\end{array}$ & $\begin{array}{l}\text { Ministère de la réforme et de l'innovation dans } \\
\text { l'administration publique, par le biais du } \\
\text { Département de la fonction publique }\end{array}$ \\
\hline Portugal & $\begin{array}{l}\text { Élite politique d'encadrement (agents } \\
\text { publics élus) }\end{array}$ & $\begin{array}{l}\text { Ministère des finances et de l'administration } \\
\text { publique }\end{array}$ \\
\hline Espagne & $\begin{array}{l}\text { Élites politiques aux trois niveaux de } \\
\text { l'administration }\end{array}$ & $\begin{array}{l}\text { Ministère des administrations publiques en } \\
\text { coordination avec les régions autonomes et les } \\
\text { entités locales }\end{array}$ \\
\hline Suisse & $\begin{array}{l}\text { Élites politiques (Parlement suisse) et } \\
\text { d'encadrement (Conseil fédéral) }\end{array}$ & \begin{tabular}{l} 
Département fédéral des finances \\
\hline
\end{tabular}
\end{tabular}

Source : OCDE (GOV) 
68. II faut alors se demander si les dirigeants économiques sont prêts à conduire le changement. II ne fait aucun doute qu'ils ont la formation technique nécessaire pour mettre en œuvre une proposition de réforme sophistiquée, comme la LOLF française ; en revanche, leur capacité à gérer le changement produit par la réforme est une autre question. Si les programmes de régionalisation et de productivité finlandais ont posé des difficultés de mise en œuvre, ça n'est pas dû au manque de connaissances techniques mais aux compétences de direction limitées et à l'absence d'une stratégie de gestion du changement. Cela peut notamment s'expliquer par le profil éducatif des gestionnaires, qui n'ont peutêtre pas été formés à la direction d'organisations, leur formation ayant plutôt mis l'accent sur des aspects techniques et non sur la manière de gérer les effets de leurs décisions ${ }^{20}$. La Finlande, la France, l'Espagne et la Suisse considèrent que le développement des compétences de leadership des gestionnaires contribue de façon déterminante à faciliter le changement. Cela signifie deux choses. Premièrement, aussi compétents et dévoués que soient les fonctionnaires, sans un leadership impliqué et de confiance, les efforts de réforme peuvent être sans effet. Deuxièmement, les gestionnaires doivent être en mesure de se comporter en dirigeants. Selon l'expérience finlandaise, le leadership est une compétence qui peut s'apprendre, d'où l'importance de la formation.

\section{Encadré 10. Recours à de véritables gestionnaires dans le secteur public}

Les premières rencontres internationales de la gestion publique, organisées par l'Institut de la Gestion Publique et du Développement Économique en 2002, ont conclu que la dimension managériale des postes à responsabilité implique nécessairement une suite de changements radicaux, comme la nomination de responsables en fonction de leurs compétences managériales. Cependant, cela ne résout pas tout. Les compétences managériale seules ne suffisent pas à produire et gérer le changement ; elles doivent être complétées par des compétences de leadership. Les gestionnaires doivent non seulement bien faire les choses, mais ils doivent également stimuler les gens. Ils doivent jouir d'une autorité morale leur permettant de compter sur la confiance des gens et d'exiger leur soutien en faveur de la concrétisation d'une vision. Sans le soutien de l'élément humain les gestionnaires sont incapables de gérer efficacement les programmes de réforme. Les compétences managériales et de leadership sont donc les deux qualités nécessaires.

69. Le facteur de la conduite du changement évoque également l'idée que les dirigeants sont non seulement des individus mais des groupes, ce qui a été illustré par les six études de cas. Les dirigeants ne sont pas seulement des individus mais aussi des groupes qui agissent sous forme de coalitions directrices ou de cause pour expliquer le besoin de réforme, mettre au point une vision d'avenir, la faire connaître à l'ensemble des parties prenantes, et obtenir et conserver un soutien social et politique. Aucune des expériences de réforme analysées ne mentionne le rôle dominant d'un seul individu dans la conduite du processus de réforme. Dans le cas de la France, par exemple, deux membres de I'Assemblée nationale ont proposé d'apporter des réformes à la LOLF et rédigé le document initial qui a été examiné, modifié et approuvé par la suite. Mais pour que la réforme soit adoptée par le Parlement, ils ont eu besoin du soutien des différents groupes politiques qui ont constitué une coalition de cause qui a conduit à la mise en œuvre de l'action de réforme.

70. Politique institutionnelle. Ce facteur a vocation à expliquer l'importance des réseaux coopératifs d'organisations. En effet, le dialogue, le soutien politique et la participation sociale à l'élaboration des politiques jouent un rôle fondamental dans la création d'un sentiment d'appartenance et dans la légitimation des propositions de réforme. Cela donne à penser que le service public est

20 Cette hypothèse exige d'être confirmée par des recherches empiriques supplémentaires. Une analyse du niveau d'éducation des responsables peut aider à savoir si les universités et les établissements d'enseignement supérieur préparent non seulement du personnel hautement formé aux aspects techniques (par exemple : économie, finances, droit) mais aussi des dirigeants capables de gérer des organisations publiques. 
intimement lié aux aspirations, aux pressions et aux aléas du monde politique. Dans les six pays analysés, les décisions sont prises au sommet de la hiérarchie de l'organisation, ce qui illustre la prédominance d'un système descendant dans le processus de formulation des politiques, les élites politiques et managériales jouant un rôle de premier plan dans la prise de décision. Les pays de l'OCDE semblent recourir à plusieurs mécanismes pour créer des réseaux de coopération formels et informels, tel que l'utilisation des médias, d'enceintes pour la consultation et/ou les alliances politiques.

71. Dans le cas de l'Espagne, par exemple, des consultations poussées avec les représentants de la société civile ont permis de parvenir à un consensus sur les objectifs de réforme et sur la manière de les atteindre, réduisant du même coup l'opposition politique à cette initiative. En France, le dialogue et la négociation au sein des différentes forces politiques a favorisé l'acceptation de la LOLF. Pendant ces processus de négociation et de dialogue, il a fallu exprimer clairement la nécessité du changement pour convaincre les parties prenantes de la nécessité d'une réforme. C'est le moment où les initiateurs d'une action de réforme doivent expliquer les risques du maintien du statu quo et les avantages du changement pour convaincre tous les acteurs concernés et obtenir et conserver leur soutien. Cela permet de penser que les élites politiques sont, dans une certaine mesure, ouvertes à de nouvelles idées applicables tant que celles-ci ne remettent pas en cause leurs privilèges.

72. Dans les pays de l'OCDE, la création de réseaux informels pour œuvrer en faveur du changement dans le secteur public témoigne d'une stratégie plus active ou participative en matière d'élaboration des politiques, ce qui démontre que le soutien politique et le soutien social sont indispensables pour garantir l'adoption d'une action de réforme. Cela dépend néanmoins d'un leadership et d'une vision idéologique efficaces, la proposition de réforme devant être suffisamment convaincante pour remporter le soutien des gens. La France, l'Italie, le Portugal et l'Espagne ont eu besoin d'intégrer des agents extérieurs au secteur public pour constituer une coalition afin de créer un consensus ou de donner de la légitimité à une décision déjà prise. En tout état de cause, une stratégie participative facilite le lancement d'un processus de réforme en impliquant tout le monde. L'enseignement à tirer de ces expériences est donc que la construction d'un réseau de coopération pour parvenir à un consensus sur la nécessité d'une réforme afin de résoudre un problème supposé exige des canaux de communication efficaces entre l'ensemble des parties prenantes concernées. Une stratégie participative d'élaboration des politiques fait montre de transparence, de fiabilité et de responsabilité et encourage ainsi le soutien social et politique en faveur de la réforme et du changement.

73. Moyens de mise en œuvre. Ce facteur a trait aux mécanismes utilisés par les dirigeants pour façonner et peser sur la mise en œuvre des actions et sur le comportement des acteurs. Les éléments d'appréciation fournis par les études de cas révèlent que la formation des gestionnaires a été un instrument communément utilisé pour gérer le changement. En général, la formation avait deux objectifs : expliquer les dimensions politiques et techniques de l'action de réforme, et encourager le dialogue horizontal et vertical entre les gestionnaires. Certains des programmes de réforme sont très complexes et les gestionnaires doivent être formés avant et pendant la mise en œuvre de l'action : c'est le cas de la Finlande, de la France, du Portugal, de l'Espagne et de la Suisse. Les stages de formation favorisent également le dialogue et le partage d'expériences entre les gestionnaires, comme dans le cas de la Finlande et de la France. Ils constituent des enceintes de discussion qui permettent de tirer des enseignements permettant d'améliorer l'action de réforme. L'une des valeurs ajoutées de la formation est qu'elle permet d'expliquer le besoin de changement de manière plus approfondie à des personnes qui sont chargées de la mise en œuvre du programme. En Finlande, le programme Finwin constitue non seulement une enceinte où l'on peut échanger des expériences, mais il a également pour mission de démontrer aux gestionnaires des différents niveaux de l'administration qu'il y a urgence à réformer. 
74. La mise en œuvre progressive de mesures de réforme a facilité la gestion du changement. Comme le résument bien les cas français et espagnol, la mise en œuvre progressive du changement favorise l'adaptation, une compréhension plus précise de la réforme, et même l'amélioration de certains aspects techniques de l'action. De plus, il semble que les pays de l'OCDE sont conscients des risques qu'ils courent en criant trop tôt victoire. II ne suffit pas, par exemple, d'obtenir un soutien politique et social, la réelle difficulté étant de le conserver pendant toute la durée du processus de réforme. Une autre solution viable, au vu de l'expérience finlandaise, française et portugaise, réside dans la formation des gestionnaires et dans une stratégie de communication.

La France, pour sa part, a testé le programme de réforme dans certains domaines du service public. Le changement suppose généralement de l'innovation, de sorte que l'expérimentation peut permettre d'améliorer l'action avant qu'elle ne soit pleinement mise en œuvre. Garvin (1993) affirme que l'expérimentation est l'un des éléments constitutifs d'un système d'apprentissage qui détermine la réussite d'un programme de réforme. La LOLF envisageant l'adoption de pratiques budgétaires innovantes, la mise à l'épreuve de ses aspects conceptuels et techniques a permis aux gestionnaires et aux fonctionnaires en général de comprendre la réforme et d'apporter les améliorations nécessaires.

\section{Évaluation des stratégies de gestion du changement}

75. Les études de cas présentées n'indiquent aucunement l'existence d'une stratégie claire de gestion du changement. Elles évoquent l'adoption, dans une certaine mesure, d'outils destinés à faciliter la mise en œuvre de mesures de réforme. Il est vrai que cela fait partie de l'objectif d'une stratégie de gestion du changement mais ces outils en eux-mêmes ne sont pas réellement en mesure d'instaurer de nouvelles valeurs dans la culture administrative sans une stratégie définie et délibérée. En ce sens, il n'est pas possible de parler de bonnes ou de mauvaises pratiques de gestion du changement mais de mesures plus ou moins intéressantes pour la mise en œuvre d'une proposition de réforme. Cela laisse supposer que les pays de l'OCDE sous-estiment combien il est important de tenir compte de la question de la gestion du changement dans les processus d'élaboration et de mise en œuvre des politiques. Qui plus est, si le but des réformes est un changement d'orientation, il est difficile dans ce cas de savoir si un changement a bien eu lieu. Une stratégie de gestion du changement doit définir un processus cohérent et complet de gestion du changement produit ou censé être produit par l'adoption d'une réforme, y compris ses effets secondaires. Le tableau 3 met en évidence les principaux avantages et les principales limitations de chacune des mesures de réforme présentées dans le présent document. Il témoigne de l'absence d'une stratégie pour faire face aux effets des réformes menées dans le service public. 
Tableau 3. Évaluation des stratégies de conduite du changement dans les pays de l'OCDE

\begin{tabular}{|c|c|c|}
\hline $\begin{array}{l}\text { Pays de } \\
\text { l'OCDE }\end{array}$ & Atouts & Faiblesses \\
\hline Finlande & $\begin{array}{l}\text { - S'attaque aux lacunes du leadership au } \\
\text { sommet de la hiérarchie managériale. } \\
\text { _ } \text { Facilite le dialogue horizontal et } \\
\text { vertical. } \\
\text { - Met l'accent sur la création d'une } \\
\text { ébauche de vision. } \\
\text { - Crée une coalition directrice en vue } \\
\text { d'une conception de l'avenir. }\end{array}$ & $\begin{array}{l}\text { - Perception négative du programme par } \\
\text { certains gestionnaires et représentants des } \\
\text { employés. } \\
\text { - } \text { Information insuffisante de tous les acteurs } \\
\text { concernés sur les buts du programme. } \\
\text { - Durée limitée du programme, aucune } \\
\text { prolongation n'étant prévue. }\end{array}$ \\
\hline France & $\begin{array}{l}\text { - } \text { Fondements politiques solides. } \\
\text { - } \quad \text { Cadre conceptuel original. } \\
\text { - } \quad \text { S'appuie sur une stratégie. } \\
\text { - } \quad \text { Envisage une stratégie de } \\
\text { communication. } \\
\text { - } \quad \text { Prévoit de piloter l'action. } \\
\text { - Envisage la formation en vue d'une } \\
\text { sensibilisation accrue aux buts de la } \\
\text { réforme. } \\
\text { - } \quad \text { Les initiateurs ont pris le temps de } \\
\text { mûrir la réforme. }\end{array}$ & $\begin{array}{l}\text { - L'originalité de la réforme fait qu'il peut être } \\
\text { difficile de la comprendre pleinement et de } \\
\text { convaincre les fonctionnaires du besoin de } \\
\text { changement. } \\
\text { _ Bien que les hommes politiques soutiennent } \\
\text { l'initiative, les fonctionnaires n'ont pas } \\
\text { participé à la définition de la réforme, ce qui } \\
\text { peut se traduire par une résistance au } \\
\text { changement. } \\
\text { - La formation des dirigeants à la conduite du } \\
\text { changement est réduite au minimum. } \\
\text { - La mise en oeuvre de la LOLF peut déclencher } \\
\text { le besoin de réformes supplémentaires dans } \\
\text { d'autres domaines de la budgétisation et des } \\
\text { finances, ce qui peut accroître la complexité de } \\
\text { la réforme. }\end{array}$ \\
\hline Italie & $\begin{array}{l}\text { - Les réformes s'appuient sur des } \\
\text { éléments d'appréciation tirés de } \\
\text { l'expérience d'autres nations de l'UE. } \\
\text { - } \text { Adopte une approche auto-critique. } \\
\text { - Fondements politiques solides. } \\
\text { - Envisage la formation continue. }\end{array}$ & $\begin{array}{l}\text { - Absence d'une vision précise de l'avenir du } \\
\text { service public qui donnerait une cohérence } \\
\text { aux efforts de réforme. } \\
\text { _ } \quad \text { Pas de mise à l'épreuve des aspects } \\
\text { techniques les plus complexes de la réforme. } \\
\text { - } \quad \text { Les mesures de réforme n'ont pas fait l'objet } \\
\text { de consultations avec les acteurs concernés } \\
\text { (comme les dirigeants syndicaux), ce qui } \\
\text { compromet le soutien en faveur de la réforme. } \\
\text { - } \quad \text { La formation ne résout pas le problème du } \\
\text { manque de leadership dans le service public } \\
\text { pour gérer le changement. }\end{array}$ \\
\hline Portugal & $\begin{array}{l}\text { - Crée une coalition de cause pour } \\
\text { soutenir la réforme (les médias, les } \\
\text { acteurs économiques et les hommes } \\
\text { politiques). } \\
\text { - } \quad \text { La formation professionnelle s'attaque } \\
\text { au manque de leadership dans le } \\
\text { service public. } \\
\text { - Donne une continuité aux efforts de } \\
\text { réforme antérieurs. } \\
\text { - Leadership politique engagé. }\end{array}$ & $\begin{array}{l}\text { - Soutien insuffisant de la part des } \\
\text { fonctionnaires qui ont le sentiment que leurs } \\
\text { intérêts sont touchés. } \\
\text { - } \\
\text { Absence d'une vision précise de l'avenir du } \\
\text { service public permettant de donner une } \\
\text { cohérence aux efforts de réforme. } \\
\text { - Les retards dans la mise en oeuvre de la } \\
\text { réforme sont dus principalement aux priorités } \\
\text { politiques et aux procédures bureaucratiques } \\
\text { et non à une stratégie destinée à favoriser } \\
\text { l'adaptation. }\end{array}$ \\
\hline
\end{tabular}


Tableau 3. Évaluation des stratégies de conduite du changement dans les pays de l'OCDE

(suite)

\begin{tabular}{|c|c|c|}
\hline $\begin{array}{l}\text { Pays de } \\
\text { l'OCDE }\end{array}$ & Atouts & Faiblesses \\
\hline Espagne & $\begin{array}{l}\text { - La réforme a fait l'objet de } \\
\text { consultations avec un grand nombre } \\
\text { d'acteurs (hommes politiques, } \\
\text { fonctionnaires et membres de la } \\
\text { société civile). } \\
\text { - Création d'une coalition de cause pour } \\
\text { voter et appliquer la nouvelle loi. } \\
\text { - Plusieurs dispositions de la réforme } \\
\text { sont testées avant leur pleine mise en } \\
\text { œuvre. }\end{array}$ & $\begin{array}{l}\text { - } \text { Très complexe. } \\
\text { - } \quad \text { Exige des réglementations et des mesures } \\
\text { spécifiques en matière de GRH au niveau } \\
\text { fédéral et dans les communautés autonomes. } \\
\text { - } \quad \text { La formation met l'accent sur les aspects } \\
\text { techniques de la réforme mais pas sur le } \\
\text { leadership. } \\
\text { - }\end{array}$ \\
\hline Suisse & 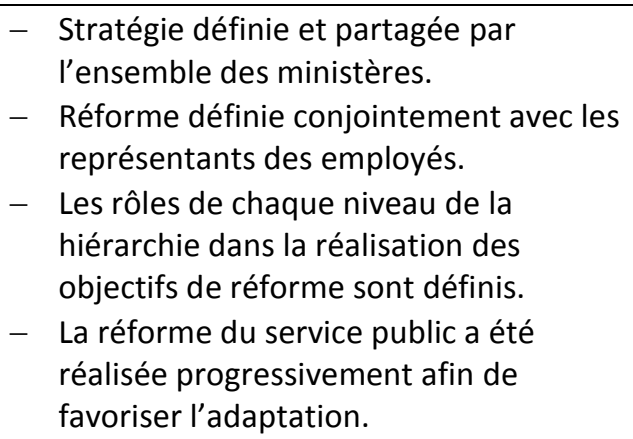 & $\begin{array}{l}\text { - Très peu d'intérêt porté aux questions de } \\
\text { leadership. } \\
\text { - Les mesures de réforme n'ont pas été testées. } \\
\text { - Aucune stratégie de communication } \\
\text { envisagée. }\end{array}$ \\
\hline
\end{tabular}

Source : OCDE (GOV).

76. Comme le montre le tableau 3, aucune des mesures de réforme ne s'accompagne d'un plan de gestion du changement. Le fait que tout programme ou action de réforme présente des atouts et des faiblesses sur le plan de la formulation des politiques n'est pas nouveau, ce qui est remarquable en revanche, c'est le peu de considération accordé à la gestion du changement et à ses effets secondaires probables. On peut se demander quel sera l'impact de l'absence de ce type de stratégie sur les résultats de la réforme. Si la gestion du changement est une variable de poids, alors pourquoi les responsables de l'élaboration des politiques et les décideurs y accordent-ils si peu de considération ? C'est probablement l'une des raisons pour lesquelles certaines réformes ne parviennent pas à atteindre leurs objectifs ; cela peut également être dû au fait que la gestion du changement ne joue pas un rôle si déterminant que cela dans la concrétisation d'un changement d'orientation. La réponse à ces questions exige cependant des recherches théoriques et empiriques supplémentaires. 


\section{REMARQUES FINALES ET PROPOSITIONS}

77. Le présent document a utilisé la notion de réceptivité pour expliquer et comprendre comment les pays de l'OCDE gèrent le changement dans l'administration. Les constatations des études de cas ont des répercussions sur la théorie et la pratique de la gestion du changement. Même si les conclusions ne sont pas censées être exhaustives, elles peuvent étayer et guider les travaux de recherche et les débats futurs dans ce domaine. Elles permettent également de suggérer certaines perspectives intéressantes de nouvelles recherches.

\section{À propos du cadre théorique}

78. La notion de réceptivité offre une manière d'aborder l'analyse du changement dans l'administration. Les quatre facteurs -- vision idéologique, conduite du changement, politique institutionnelle et moyens de mise en œuvre -- facilitent la compréhension du changement car ils permettent une analyse schématique et intègrent la plupart des éléments examinés dans les travaux publiés sur la gestion du changement. Ce cadre analytique permet d'expliquer des cas de réforme issus de contextes et domaines d'action différents, car il ne suppose pas l'utilisation d'une base idéologique, et ne spécifie pas non plus I'utilisation de certaines hypothèses. De ce fait, ce cadre peut s'avérer utile pour expliquer le changement dans les organisations publiques comme dans les organisations privées.

79. Cependant, la notion de réceptivité ne peut expliquer seule la concrétisation du changement. En effet, pour expliquer ou estimer si le changement a réellement eu lieu et dans quelle mesure, la notion de réceptivité requiert la méthode d'analyse historique comparative. Il faut du temps pour que le changement se produise; ainsi, la manière la plus efficace d'en apporter la preuve est d'analyser l'évolution d'une action de réforme et ses effets dans le temps. Une méthode historique comparative offrirait les éléments nécessaires pour vérifier si la culture managériale est une autre solution viable pour transformer le service public en un organisme plus efficient et efficace. C'est un aspect qu'il faudrait associer à l'évaluation de l'action et qui exigera des travaux de recherche théorique et empirique supplémentaires. De plus, cela donne plus de poids à l'argument selon lequel une stratégie de gestion parallèle devrait accompagner tout le cycle de l'action, car c'est un élément crucial du processus d'élaboration des politiques.

\section{À propos de la pratique de la gestion du changement dans les pays de l'OCDE}

80. Pour que le changement se produise dans l'administration, il faut une transformation de la culture et des attitudes. La mise en oeuvre d'une action de réforme entraîne invariablement l'opposition de personnes qui ont le sentiment que leurs intérêts sont touchés et qu'elle va à l'encontre de leurs valeurs. Ainsi, le fait de maîtriser la résistance et d'obtenir et de conserver le soutien des gens à l'égard d'une action de réforme témoigne d'une gestion efficace du changement. Le leadership semble jouer un rôle crucial dans la concrétisation du changement en ayant une influence morale sur les gens et en s'assurant de leur implication et de leur soutien.

81. Le tableau 4 présente les éléments ou facteurs qui permettent aux pays de l'OCDE de gérer le changement. Ils confirment les conclusions déjà formulées dans les travaux publiés (Garvin, 1993 ; Drucker, 1995 ; Kotter, 1995 ; Whiteley, 1995 ; Hushion, 1999 ; Driscoll et Morris, 2001 ; Baulcomb, 2003 ; Brunsson, 2006) et en ce sens d'aucuns diront qu'ils n'ajoutent pas grand-chose aux connaissances sur le domaine. Cependant, ils révèlent que la gestion du changement dans le secteur public est tout aussi importante que la gestion du changement dans le privé. Qui plus est, la gestion du 
changement dans les organisations publiques est plus complexe car elle fait intervenir un éventail plus large d'acteurs (publics et privés) et quels que soient ses résultats, ils ont des répercussions sur la société dans son ensemble.

82. Il est un aspect important qu'il faut souligner, à savoir le fait que les pays de l'OCDE sousestiment l'importance de la gestion du changement et son rôle dans l'élaboration des politiques. La manière dont les pays de l'OCDE gèrent le changement, comme l'illustre le tableau 4, témoigne du fait que les instruments utilisés correspondent plus une méthode casuistique de formulation des politiques qu'à une stratégie délibérée de gestion des effets de la mise en œuvre d'une proposition de réforme. II n'a pas été possible de prouver l'existence d'une stratégie globale. La politique ne peut se substituer à la gestion du changement, dont elle constitue seulement un aspect. De la même manière, une réforme élaborée ne suffit pas à garantir la concrétisation du changement car, par exemple, plus d'éléments d'appréciation ne conduisent pas nécessairement à de meilleures politiques. Ce problème peut être dû à l'absence d'une méthodologie ou du moins de principes directeurs en matière de gestion du changement. Il est un autre aspect qu'il convient de souligner, à savoir le fait que le secteur public semble être confronté à un problème de leadership. Les enseignements tirés des six expériences sont que dans les pays de l'OCDE les hauts responsables ne sont pas nécessairement de bons dirigeants, pourtant pour obtenir du changement ils doivent assumer les deux rôles. Par conséquent, il est capital de consolider les compétences en leadership des gestionnaires pour que le changement se produise. Le problème est accentué par la faible légitimité et le peu de confiance que leur accordent les citoyens (OCDE, 2001, 2006). Cela compromet, par exemple, l'introduction d'une culture orientée vers les performances dans le service public, la confiance à l'égard des gestionnaires étant capitale pour sa réussite. De plus, les informations disponibles ne permettent pas de déterminer ou d'estimer si le changement a véritablement lieu dans l'administration. Comme cela a été indiqué plus tôt dans le présent document, la concrétisation du changement est un processus de longue haleine ; il faudra donc analyser ces six expériences de réforme plus tard, car elles n'en sont encore qu'à leurs débuts, pour vérifier si les réformes administratives ont eu l'effet attendu sur la culture du service public. Si la notion de réceptivité doit être utilisée, elle devra inclure des éléments qui confirment la concrétisation du changement et pas seulement la manière dont il se produit et dont il est géré. 
Tableau 4. Enseignements tirés des expériences des pays de l’OCDE en matière de gestion du changement

\begin{tabular}{|c|c|}
\hline Pays de l'OCDE & Principaux enseignements en matière de gestion du changement \\
\hline Finlande & $\begin{array}{ll}\checkmark & \text { L'absence d'une stratégie de communication et d'une vision d'avenir commune sape et compromet les } \\
& \text { efforts de réforme. } \\
\checkmark & \text { La communication et le dialogue entre l'ensemble des parties prenantes dès les premiers stades de la } \\
\checkmark & \text { réforme sont de la plus haute importance pour la concrétisation du changement. } \\
\checkmark & \text { Un leadership impliqué est indispensable à la concrétisation du changement. } \\
\checkmark & \text { Les hauts fonctionnaires doivent être en mesure d'assumer les rôles de gestionnaires et de dirigeants. }\end{array}$ \\
\hline France & 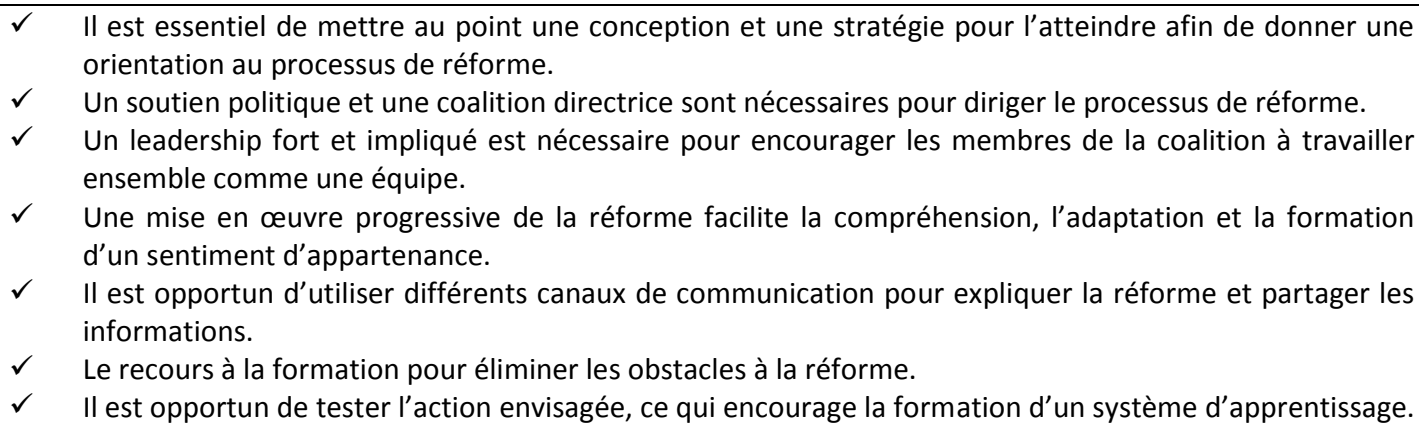 \\
\hline Italie & $\begin{array}{ll}\checkmark & \text { Les examens par les pairs et l'évaluation comparative constituent des moteurs externes du changement. } \\
\checkmark & \text { La production du changement prend beaucoup de temps et il faut veiller à entretenir l'implication } \\
& \text { politique pendant toute la durée du processus de réforme. } \\
\checkmark & \text { La gestion du changement exige une part d'auto-critique. }\end{array}$ \\
\hline Portugal & $\begin{array}{l}\checkmark \quad \text { Pour maintenir la dynamique et l'efficacité de la réforme, il est nécessaire d'adapter et de réactualiser } \\
\text { en permanence les réformes en cours. } \\
\checkmark \quad \text { Une mise en œuvre progressive des mesures de réformes accroît les possibilités d'une intégration réelle } \\
\text { du changement dans la culture du service public. }\end{array}$ \\
\hline Espagne & $\begin{array}{ll} & \text { Le dialogue avec différentes forces sociales et politiques et leur intégration débouchent sur une stratégie } \\
\checkmark & \text { commune et sur l'implication. } \\
\checkmark & \text { Une mise en œuvre progressive de la réforme accroît la réceptivité au changement. } \\
\text { particuliers pour faciliter l'acceptation et la compréhension. }\end{array}$ \\
\hline Suisse & 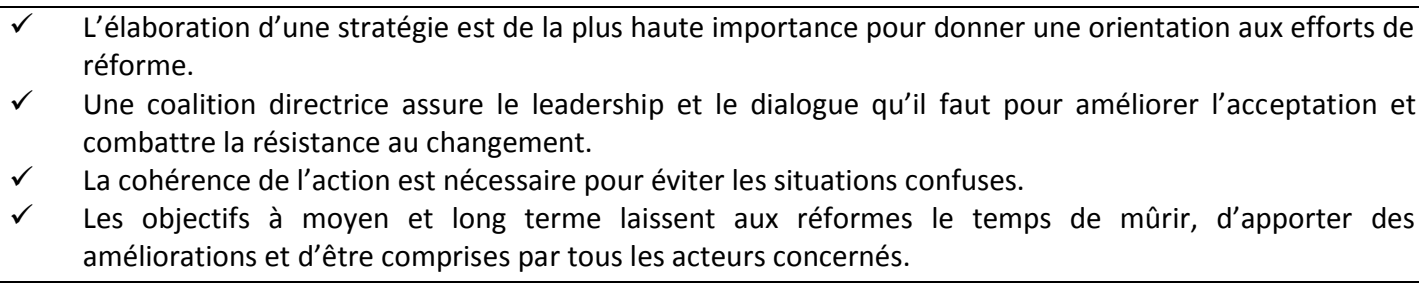 \\
\hline
\end{tabular}

Source : OCDE (GOV)

\section{Propositions}

83. S'appuyant sur une combinaison des expériences des six pays de l'OCDE et sur les éléments d'appréciation relevés dans les travaux publiés, le présent document laisse entendre qu'une réforme, en particulier une réforme administrative, doit avoir les attributs suivants pour être gérable ou, en d'autres termes, être plus facile à entreprendre, et pour pouvoir atteindre plus facilement ses objectifs.

- Elle doit être simple. Une proposition de réforme ne peut refléter toute la complexité du service public. Les propositions de réforme expriment des principes ou des opinions en vue d'améliorer une situation ; partant, elles doivent éviter d'inclure des informations détaillées sur des questions techniques. Lorsque les propositions de réforme sont trop complexes, elles ont plus de chances d'entraîner des malentendus et en conséquence une résistance au changement. 
- Elle doit être cohérente. Cela signifie que la réforme doit invoquer un unique système de valeurs et de principes (Brunsson, 2006), ce qui est particulièrement important sachant que les propositions de réforme ont tendance à modifier les fondements culturels d'une organisation. $\mathrm{Ce}$ sont les raisons pour lesquelles les réformes constituent une manière particulière d'envisager les problèmes. De plus, il n'est ni possible ni opportun d'entreprendre plusieurs réformes en même temps pour traiter le même problème.

- Elle doit s'appuyer sur des éléments d'appréciation. Une réforme doit avancer des arguments convaincants sur un problème, présent ou futur, et sa solution. Pour ce faire, on peut notamment tirer des enseignements d'autres expériences, internes et externes, ce qui exige de se lancer dans une démarche d'exploitation des enseignements (Rose, 2005). Cependant, il faut envisager la possibilité que les expériences réussies dans certains contextes ne produiront pas nécessairement les mêmes résultats dans d'autres. La recherche d'éléments d'appréciation doit s'intéresser aussi bien à des cas de réussite qu'à des cas d'échec car il est possible de tirer des enseignements d'expériences négatives, et elle doit tenir compte des différences culturelles et contextuelles entre les pays.

- Elle doit être réaliste. Généralement les ambitions des réformes sur le plan de leurs objectifs et de ce qu'elles peuvent réellement obtenir dans un contexte et une période spécifique sont simples. Les réformes doivent être viables sur le plan politique et technique. Ainsi, les responsables de l'élaboration des politiques et les décideurs doivent se demander ce qu'ils peuvent réellement obtenir et sur quelle période au vu des atouts et des limites de la proposition de réforme. C'est d'une importance capitale si l'on ne veut pas perdre le soutien à la réforme en suscitant de faux espoirs.

- Elle doit prévoir un plan d'action pour faire face aux effets secondaires éventuels. Les réformes sont un jeu de hasard, dans une certaine mesure, car elles ne sont pas sans risque et peuvent avoir des effets secondaires. La réforme d'un aspect de la structure administrative peut avoir des incidences sur d'autres aspects. Par exemple, l'évaluation des performances peut être un moyen d'améliorer l'efficacité du service public mais peut engendrer un système de favoritisme si des mesures adaptées ne sont pas prises pour l'éviter. En effet, les réformes ont leurs limites et elles ne peuvent certainement pas tout résoudre; par ailleurs les réformes peuvent entraîner d'autres réformes. Ceci étant, il faut reconnaître que les effets secondaires ne sont pas tous négatifs, et qu'il peut également y avoir des conséquences positives.

- Elle doit mettre l'accent sur l'avenir. La réforme des organisations publiques est un processus de longue haleine et l'on ne peut s'attendre à des résultats immédiats. La persévérance des dirigeants est nécessaire si l'on veut que les réformes aient lieu et que des changements se concrétisent, car les réformes promettent des avantages futurs qui parfois ne seront palpables qu'une fois le processus de mise en œuvre achevé (Brunsson, 2006).

84. Ces attributs peuvent faciliter à la fois la gestion d'une réforme et la gestion du changement. Cela tient au fait qu'une réforme simple, cohérente, fondée sur des éléments d'appréciation, réaliste et qui propose des solutions sur le long terme est relativement plus facile à expliquer et à comprendre. Ces éléments pourraient probablement être développés plus avant dans les travaux publiés sur l'élaboration des politiques car il faudrait qu'ils fassent partie intégrante du processus de formulation des politiques. Cela peut également sembler évident, mais le fait de faire une distinction entre ce qui caractérise une réforme gérable et une réforme qui ne l'est pas et de l'utiliser comme point de départ pour définir la réforme peut limiter le niveau de risque. 
85. Les études de cas donnent à penser que le processus d'élaboration des politiques devrait être complété par une stratégie de gestion du changement. C'est particulièrement important car, comme nous l'avons vu au début du présent document, la réforme et le changement sont deux notions différentes mais étroitement liées. Les enseignements tirés de l'expérience des six pays de l'OCDE analysées dans le présent document peuvent servir de point de départ pour la mise au point d'une méthodologie de gestion du changement en dépit de leurs différences de contenu et de contexte. La méthodologie, loin d'être normative, devrait servir de référence pour l'évaluation de la réussite et de l'échec d'une politique. La mise au point d'une telle méthodologie pourrait être orientée par les questions et considérations suivantes :

1. Le besoin de changement a-t-il été soigneusement établi ? Comment a-t-il été défini ?

- Il est nécessaire d'identifier une crise ou une crise potentielle ou du moins des perspectives intéressantes d'amélioration avant d'entreprendre une réforme.

- Des facteurs externes et internes peuvent conduire à l'adoption d'une réforme.

2. Existe-t-il une vision d'avenir qui donne une orientation aux efforts de réforme ? Est-elle partagée par tous ceux qui participent au processus de réforme et par les membres de la collectivité ?

- Une vision d'avenir donne une cohérence aux efforts de réforme et reflète l'idéologie dominante.

- La vision doit être portée à la connaissance d'un maximum d'acteurs participant au processus de réforme ou concernés.

- Le partage d'informations permettrait à tous de rester impliqués et contribuerait à rendre la réforme compréhensible.

- Les propositions de réforme doivent être compatibles avec la culture de l'organisation, de sorte qu'il faut être particulièrement prudent lorsque l'on tire des enseignements d'autres contextes.

3. Les membres de la société ont-ils eu la possibilité d'exprimer leur opinion sur la proposition de réforme ? Voient-ils le même problème et approuvent-ils la solution proposée ?

- Les membres de la société (universitaires, dirigeants syndicaux, entrepreneurs, etc.) doivent être consultés pour enrichir ou améliorer la proposition de réforme.

- La consultation donne de la légitimité l'action de reforme et favorise son soutien.

- Les fonctionnaires en général doivent être consultés au sujet de la faisabilité de l'action proposée et il faut les intégrer au processus de formulation pour obtenir leur soutien.

4. Existe-t-il une coalition directrice (leadership) disposant d'un pouvoir moral et politique suffisant pour diriger l'effort de réforme?

- Un leadership réel peut réduire la résistance comportementale au changement, qui est un problème permanent.

- Pour que le changement se produise, il faut qu'une coalition directrice et de cause intégrant des acteurs issus de l'intérieur et de l'extérieur du service public dirige les efforts de réforme.

- Le but de la coalition directrice doit être d'encourager l'ensemble des acteurs concernés à œuvrer en faveur de la concrétisation de la stratégie.

- La coalition directrice doit identifier les obstacles au changement et s'efforcer de les éliminer. 
5. La proposition de réforme est-elle mise en œuvre progressivement pour favoriser l'adaptation et l'amélioration ? La réforme a-t-elle été testée ?

- La mise en œuvre progressive d'une réforme favorise l'adaptation, laquelle est indispensable à la concrétisation du changement.

- La mise à l'épreuve de la réforme permet d'améliorer les aspects techniques de l'action, et facilite la familiarisation avec la proposition de réforme.

6. Les ressources disponibles sont-elles suffisantes pour mettre en œuvre la réforme et obtenir les effets souhaités?

- La mise en œuvre d'une réforme exige certaines compétences et ressources (financières, politiques, humaines, en connaissances et en temps).

- Les connaissances et le temps sont indispensables à la concrétisation du changement. L'innovation est capitale pour éviter de reproduire de vieilles pratiques. Le temps est essentiel pour institutionnaliser les nouvelles dispositions et mûrir la réforme.

7. Les effets secondaires de la réforme ont-ils été pris en compte ? Les initiateurs de la réforme sont-ils conscients des limites de la réforme?

- Les réformes ne sont pas sans risque et peuvent avoir des conséquences inattendues. Partant, une stratégie de gestion du changement doit envisager des modes d'action de nature à traiter les effets secondaires négatifs.

- Une stratégie de gestion du changement doit viser à réduire le risque et à traiter les effets inattendus.

- Un projet de réforme a ses limites et ne peut vraisemblablement pas résoudre tous les problèmes, d'où la nécessité de faire preuve de réalisme quant à ce que la réforme peut réellement apporter. Cela a des incidences sur l'obtention et le maintien du soutien politique et social.

8. Le programme de réforme est-il évalué régulièrement pour améliorer ses aspects techniques et voir s'il produit les résultats souhaités ?

- L'amélioration exige une volonté d'apprentissage et de production d'informations et de connaissances.

- Les organisations publiques doivent être en mesure de créer, d'acquérir et de transférer des connaissances, et d'adapter leur comportement en conséquence.

86. Ces questions ont pour seul but de fournir quelques lignes directrices pour la mise au point d'une méthodologie de gestion du changement. Qui plus est, des questions de ce type peuvent guider la mise en place d'une stratégie de gestion du changement. Au vu des études de cas, on peut avancer qu'une stratégie de gestion du changement doit comporter une part d'expérimentation, de formation et d'amélioration continue, de communication et d'évaluation constante. Cependant, d'autres travaux de recherche théoriques et empiriques sur le sujet sont nécessaires.

\section{La route à suivre en matière de gestion du changement}

87. La mise au point d'une méthodologie de gestion du changement sur une base empirique générale est capitale pour orienter les travaux des responsables de l'élaboration des politiques et des décideurs. Il est également utile de noter qu'aucune des six expériences analysées dans le présent document n'indiquait comment déterminer si un changement s'était réellement produit. Aucun instrument ou paramètre ne permet véritablement de constater que de réels changements 
comportementaux ou culturels ont été engendrés par l'adoption de stratégies de gestion de la performance dans le service public. Il s'agit d'un problème à la fois théorique et pratique. Les gouvernements participent semble-t-il à des processus de changement sans quasiment se soucier de la manière d'évaluer l'impact qu'une action de réforme a eu dans la culture du service public. La manière de mesurer la réussite est un élément important pour l'évaluation des politiques mais aussi pour le maintien d'un soutien politique et social. Cela tient peut-être entre autres au fait que, comme le soutient Sinner (2004), la mise en œuvre d'un processus d'évaluation est une activité complexe influencée par des questions de pouvoir, de politique et de jugements de valeur. Même si le changement culturel est une question qualitative qui relève fortement de la perception, de futures études sur la gestion du changement auront peut-être besoin d'informations quantitatives pour parvenir à établir des généralisations. Par conséquent, une méthodologie de recherche nuancée permettrait de parvenir à des conclusions plus solides. Les futurs travaux de recherche dans le domaine peuvent avoir besoin de répondre à des questions telles que celles-ci : Comment déterminer si une action de réforme produit les résultats souhaités? Comment maintenir l'opinion positive que les citoyens ont des projets politiques et du leadership sans preuves tangibles d'une évolution positive de l'action publique? Comment évaluer les mesures en faveur de l'évolution de l'action publique en écartant toute considération politique ? L'évaluation des projets de réforme de l'action est capitale pour parvenir à une convergence de vues et à un consensus en faveur de l'institutionnalisation du changement et de la production de changements supplémentaires. Ce thème exige cependant des travaux de recherche théorique et empirique plus poussés, tout le monde n'ayant pas la même conception de la réussite de l'action publique. 


\section{PARTIE III -- LES ÉLÉMENTS D’APPRÉCIATION : ÉTUDES DE CAS SUR LA GESTION DU CHANGEMENT}

\section{FINLANDE - LE CAS DU PROGRAMME FINWIN}

\section{Introduction}

88. La Finlande a commencé à mettre en œuvre des mesures de réforme destinées à affronter les difficultés liées à une population vieillissante. Ce faisant le pays s'est néanmoins heurté à des problèmes tels qu'une communication, une convergence de vues et une stratégie commune insuffisantes à l'intérieur du secteur public, qui l'ont empêché de se préparer harmonieusement à faire face à la perte imminente de personnel qualifié. Cette étude de cas vise à analyser de quelle manière la Finlande prépare le terrain pour mettre en œuvre le changement en remédiant au manque de leadership et de communication dans le service public et, ensuite, à tirer des enseignements son expérience.

\section{La force motrice -la population vieillissante de la Finlande}

89. La Finlande est le pays de l'Union européenne où la population vieillit le plus vite, ce qui a engendré un certain nombre de difficultés pour le pays, en particulier dans le service public. En 2015 une grande partie des hauts fonctionnaires sera retraitée, ce qui se traduira par une érosion de l'expérience et des compétences nécessaires au fonctionnement du service public. Le nombre de personnes âgées de plus de 65 ans devrait augmenter de $57 \%$ d'ici 2020 et le groupe des personnes âgées de 20 à 49 ans devrait diminuer de $20 \%$ sur la même période. L'administration centrale emploie $5.2 \%$ du total de la main-d'œuvre âgée de 42.9 ans en moyenne, soit deux ans de plus que la moyenne de la population active dans son ensemble. Des départs en retraite à grande échelle commenceront plus tôt dans la fonction publique, au niveau de l'administration centrale, que dans la population active dans son ensemble. ${ }^{21}$ Ce scénario constitue un immense défi pour la fonction publique sur le plan de la gestion des ressources humaines et de la prestation des services publics.

90. Une fonction publique vieillissante peut être considérée comme une chance de mieux adapter les services publics aux besoins d'une population vieillissante, de réorganiser la main-d'œuvre et de réduire les effectifs du personnel le cas échéant. Cependant, cela pose un problème de maintien des capacités dans certains domaines stratégiques en dépit de la perte attendue de personnel compétent et expérimenté. C'est pourquoi l'administration finlandaise a mis en œuvre des programmes de régionalisation et de productivité pour faire un meilleur usage de ses ressources humaines.

21 Pour de plus amples informations voir OCDE (2007c). 


\section{Affronter les difficultés liées à une fonction publique vieillissante - deux mesures de réforme}

91. Le programme de régionalisation vise à délocaliser les fonctions de l'État dans d'autres zones du pays d'ici 2015, ce qui se traduira par le déplacement d'environ $10 \%$ de l'ensemble des fonctions de l'État en dehors de la capitale. Des centres de service distincts ont été créés dans les régions en fonction des nouveaux services demandés par la population et des besoins des services publics. II a été demandé au personnel existant de poursuivre son travail dans les centres de service. Néanmoins, les pouvoirs publics ne peuvent contraindre les fonctionnaires à changer de résidence et le programme de régionalisation n'a eu qu'un faible écho au sein du personnel. Cela a engendré des problèmes de gestion des ressources humaines et une pénurie de leadership à plus grande échelle.

92. En raison du développement démographique et de ses répercussions négatives sur le service public, le programme de productivité a pour but de réformer les fonctions de l'État afin que dans la pratique elles exigent moins de personnel, étant donné l'aggravation prévue de la diminution du personnel qualifié. L'idée maîtresse du programme est que les ministères et les agences se préparent à l'évolution des effectifs à venir. Cependant, le programme a été considéré par certains comme une mesure de réduction des effectifs. II s'est donc avéré nécessaire d'accroître le sens de la gestion du changement et du leadership.

93. La mise en œuvre de ces programmes de réforme, si elle s'est déroulée comme prévu, a également été confrontée à des difficultés en raison d'une argumentation, d'une communication et d'un débat inadaptés avec l'ensemble des acteurs concernés, ce qui a engendré un manque d'informations, de compréhension et de vision. À titre d'exemple, les parties prenantes n'étaient pas d'accord sur la quantité de personnel à supprimer et les hauts responsables n'étaient parfois pas assez impliqués dans les actions.

\section{Traiter le problème du manque de leadership - le programme Finwin}

94. Les autorités finlandaises ont réalisé qu'il était nécessaire d'accroître la diffusion des informations sur les moyens et outils concrets de gestion du changement pour préserver la mise en œuvre des programmes. Partant, en décembre 2006, la commission ministérielle chargée de la politique économique a mis en place le programme de gestion du changement intitulé Finwin - vers un nouveau leadership. Son but est de favoriser une communauté de vues et une vision commune des défis futurs et de la manière de les gérer. En d'autres termes, le programme Finwin prétend faciliter la mise en œuvre du changement par la diffusion d'informations et l'échange d'enseignements positifs et négatifs sur les programmes de réforme dans différents domaines et à différents niveaux de l'administration.

95. Le programme Finwin est destiné aux hauts fonctionnaires et aux représentants des employés. II a commencé en juin 2007 et il devrait s'achever en mai 2008, quelque 1200 personnes devant y participer. Le programme s'organise autour de séminaires qui permettent de débattre de sujets tels que le bien-être au travail, les innovations sociales, les fonctions du secteur public et la réforme de l'administration régionale. II prévoit également d'autres méthodes de travail telles que les " learning cafés » et les ateliers d'apprentissage qui complètent les débats des séminaires.

96. Cette action en faveur du leadership vise avant tout à favoriser une communauté de vues et une conception commune de l'avenir dans 150 ministères et agences qui appartiennent à des branches administratives spécifiques et jouissent d'une grande indépendance de fonctionnement. Le message est que tous les efforts doivent tendre vers le même but. Le programme Finwin se concentre en particulier sur la formation des hauts fonctionnaires à la gestion du changement. Son but est de leur fournir les 
compétences de communication et de leadership dont ils ont besoin pour affronter un environnement changeant au titre d'une stratégie commune.

97. L'un des avantages du programme est qu'il facilite le dialogue horizontal. Les hauts responsables des différents ministères et des différentes agences ont la possibilité de débattre d'une conception commune de l'orientation de leur action, de ce qu'ils veulent pour l'avenir, de leur situation dans 10 ans, et du niveau de qualité souhaité pour leurs activités. Les séminaires leur donnent l'occasion de partager leurs expériences et leurs craintes au sujet de l'avenir et des défis futurs.

\section{La perception du programme par le public}

98. Le programme Finwin est géré par le Ministère des finances avec l'aide d'une entreprise de conseil privée. De plus, un groupe de travail intersectoriel et un groupe directeur ont été créés dès le départ pour assurer la coordination du programme. Le groupe de travail, qui se réunit toutes les deux semaines, se compose de représentants du Ministère des finances, du Trésor public, de l'administration finlandaise des routes et d'un représentant des employés. D'autre part, le groupe directeur, qui se réunit une fois par mois, regroupe des directeurs de haut niveau issus de différents organismes publics et le président d'une association, et est présidé par le secrétaire permanent du Ministère de l'environnement.

99. Pendant son déroulement le programme s'est heurté à une certaine résistance, quoique non généralisée, certains hauts responsables et représentants des employés le percevant comme une "couverture" pour le programme de productivité. Le programme Finwin ayant été créé par le Ministère des finances, il a peut-être été vu comme une intervention et comme un instrument de contrôle plutôt que comme un outil destiné à aider les agences et les ministères à gérer le changement. L'absence de bien des directeurs de haut niveau à certains séminaires témoigne peut-être d'un certain scepticisme et du peu d'implication en faveur de cette action. Une fois encore, la communication a été sous-estimée. Il est alors devenu évident pour les autorités finlandaises qu'une communication claire et adaptée était indispensable à la gestion du changement.

100. Grâce à des sujets débattus lors de séminaires tel que le bien-être au travail, le programme a suscité un grand intérêt général. La participation active des employés et des représentants de syndicats aux étapes préparatoires et aux séminaires a joué un rôle décisif. En faisant des gens des partenaires actifs de l'exercice, on a favorisé l'acceptation du programme. Le soutien politique, le soutien des syndicats ainsi que des ressources financières suffisantes ont également joué un rôle fondamental dans la mise en œuvre du programme Finwin.

101. La réussite du programme Finwin dépend de son impact positif sur la mise en œuvre des projets de changement, tel que le programme de régionalisation. Cela dépend d'un certain nombre de paramètres qui varient d'un cas à l'autre, de sorte qu'il ne peut s'agir que d'un jugement d'ordre général. L'évaluation du niveau de réussite du programme s'appuiera sur les informations en retour collectées lors de chaque séminaire et des entretiens avec les acteurs de premier plan. Certains ont préconisé la poursuite du programme d'une manière ou d'une autre, mais les modalités n'ont pas encore été déterminées.

102. L'un des résultats concrets du programme Finwin devrait être l'élaboration d'un programme en cinq points pour une meilleure gestion. Au début du programme, le Ministre des finances a demandé aux participants d'élaborer un programme en cinq points pour l'amélioration de la gestion incluant, entre autres, la mise au point de mécanismes et une meilleure utilisation des $\mathrm{Tl}$, le développement de structures et d'organisations, l'amélioration de la gestion de l'âge et le transfert de 
connaissances aux jeunes, l'accent mis sur le recrutement et l'image de l'employeur, entre autres. II faut que ce programme soit concret et sa création exige l'implication des participants.

\section{En conclusion -- quels sont les enseignements pour la gestion du changement ?}

103. La mise en œuvre des programmes de régionalisation et de productivité et le lancement du programme Finwin pour contribuer à leur réussite offrent des enseignements utiles en matière de gestion du changement. Premièrement, ils montrent les dangers et les conséquences de l'absence d'une stratégie de communication et d'une vision d'avenir lorsque l'on recherche le changement. La vision d'avenir devrait donner aux gestionnaires et aux gens une idée de l'orientation à prendre, ce qui n'a pas toujours été le cas en Finlande. II faut mettre au point une stratégie de communication parallèlement à la politique de réforme afin que toutes les parties prenantes puissent disposer d'informations en temps opportun et pour éviter les malentendus et les sentiments négatifs. Les employés ne sont disposés à faire des sacrifices tels qu'un changement de résidence que s'ils sont convaincus qu'un changement bénéfique est possible.

104. Deuxièmement, l'expérience finlandaise montre également qu'un dialogue entre l'ensemble des parties prenantes tout au long du processus de changement est nécessaire. La communication et le dialogue sont deux facteurs étroitement liés. Des canaux de communication qui fonctionnent bien contribuent au dialogue. Un dialogue et une communication appropriés dès le début du processus de formulation des politiques sont des conditions préalables importantes pour la mise en œuvre.

105. Troisièmement, la mise en œuvre des programmes de régionalisation et de productivité met en évidence le rôle important du leadership dans la concrétisation du changement. Les gestionnaires n'ont pas été suffisamment préparés à assumer leur nouveau rôle en tant que responsables du processus de changement. Les hauts responsables ne sont pas nécessairement les auteurs des mesures de réforme mais ils jouent un rôle décisif dans leur mise en œuvre; ils doivent donc comprendre le processus de changement et s'attacher à le conduire. De plus, les hauts responsables doivent disposer des outils nécessaires pour gérer le processus de changement.

106. Enfin, l'expérience finlandaise montre que les hauts fonctionnaires doivent être à la fois des gestionnaires et des dirigeants. En tant que gestionnaires ils doivent minimiser le risque lorsqu'ils mettent en œuvre de nouveaux programmes et en tant que dirigeants ils doivent instaurer les bases d'une nouvelle culture dans le service public. On peut aussi avancer que, indirectement, le programme Finwin donne naissance à une coalition directrice visant à restructurer et mettre en œuvre la stratégie destinée à affronter les difficultés liées à une population vieillissante. 


\section{LA FRANCE - LE CAS DE LA LOI ORGANIQUE RELATIVE AUX LOIS DE FINANCE}

\section{Introduction}

107. En 2001 la France a entrepris une vaste réforme de la gestion des finances publiques. La Loi organique relative aux lois de finance (LOLF) a mis fin à un cadre réglementaire ancien et a instauré une nouvelle logique budgétaire qui représente, dans une large mesure, un changement culturel dans le secteur public français. Les objectifs de la présente étude de cas sont d'examiner de quelle manière le changement est géré et intégré dans la culture du secteur public, et d'esquisser quelques enseignements pour la gestion du changement dans le service public.

\section{La LOLF - une nouvelle culture budgétaire}

108. Pendant plus de 40 ans les règles et procédures de gestion du budget et des dépenses publiques de la France ont été fixées par ce que l'on appelle l'ordonnance organique de 1959. Ce cadre réglementaire n'a quasiment pas été modifié pendant toute sa période d'application ${ }^{22}$. En 1998, l'Assemblée nationale a constitué un groupe de travail chargé d'examiner l'efficacité budgétaire et le contrôle du Parlement sur les dépenses publiques. Il a conclu que le Parlement participait peu à l'amélioration de l'efficacité des dépenses publiques, et qu'il avait peu de moyens à sa disposition pour favoriser la transparence du processus budgétaire et évaluer ses performances. De plus, la fragmentation du budget en plus de 800 articles était désormais jugée inutile. Le souhait d'instaurer l'obligation de rendre des comptes et une culture orientée vers les performances dans le service public et la nécessité d'octroyer au Parlement un rôle plus actif dans le processus budgétaire a conduit à la promulgation de la LOLF.

109. La LOLF est le produit d'une négociation et d'un accord politiques entre les différentes forces politiques représentées à l'Assemblée nationale, ce qui est considéré comme un "petit miracle » en politique française. Le nouveau cadre réglementaire financier instaure une logique budgétaire selon laquelle le budget est établi sur la base de missions, de programmes et d'actions. Il établit un processus budgétaire axé sur deux éléments clés : i) la logique de la gestion de la performance publique, et ii) la transparence des informations budgétaires qui permet au Parlement d'avoir un contrôle sur le processus. La LOFL est destinée à réduire les dépenses de l'État, notamment la facture des salaires, afin d'alléger la dette publique.

110. La logique de la gestion de la performance publique est d'instaurer une culture budgétaire centrée sur les résultats plutôt qu'une logique de moyens, ce qui constituait l'une des plus grandes pierres d'achoppement dans la réglementation de 1959. Ainsi les administrateurs doivent rendre compte de l'utilisation des ressources financières qui leur sont affectées pour fonctionner. Ce faisant, deux projets sont mis en œuvre, les projets annuels de performance (PAP) qui présentent les activités des différentes administrations pour l'année suivante, et les rapports annuels de performance (RAP) qui évaluent les objectifs de l'année suivante. L'idée de base est de séparer les fonctions de l'État de ses structures en fixant des missions.

22 Le cadre réglementaire financier de 1959 a fait l'objet de plus de 38 propositions de réforme au cours de la période de 40 ans ; elles ont toutes été rejetées. 
111. La LOLF accroît la transparence budgétaire et renforce le contrôle du Parlement sur les finances publiques. Elle impose aux pouvoirs publics de présenter au Parlement, lors de l'introduction du projet de loi de finances, un rapport sur les perspectives économiques, sociales et financières pour les quatre années à venir. Cette mesure devrait accroître la transparence dans la gestion budgétaire. Suivant cette logique, le Parlement est mieux informé et joue un rôle actif dans l'affectation du budget car il est habilité à modifier la répartition des fonds entre les programmes.

\section{Préparation du terrain en vue du changement}

112. La LOLF représente le passage d'une culture de procédures à une culture de résultats. D'aucuns soutiennent que la LOLF est le fait de visionnaires qui ont conceptualisé un nouveau cadre réglementaire pour une gestion plus transparente, plus rationnelle et efficace des fonds publics ${ }^{23}$. Le Parlement a adopté la LOLF le $1^{\mathrm{er}}$ août 2001 et a accordé un délai de cinq ans pour préparer le terrain avant la présentation du premier budget selon la LOLF. La stratégie de mise en œuvre comportait quatre éléments principaux : a) mise au point du cadre conceptuel; b) information/communication ; c) expérimentation; et d) formation.

113. Avec l'introduction de la LOLF, il a fallu mettre au point un nouveau cadre conceptuel. En effet, les concepts, les outils et les procédures nécessaires à la mise en en œuvre de la LOLF étaient inexistants. Les autorités françaises ont donc dû instituer un concept original de la performance qui donnait plus d'importance à l'efficacité, la qualité et l'impact sur la société ${ }^{24}$. Tous les objectifs et indicateurs doivent être orientés vers la réalisation de ces éléments. De plus, il a fallu concevoir un nouveau système comptable et par-dessus tout une nouvelle relation entre l'État et le Parlement. II a également fallu nommer un chef de projet, le directeur de la réforme budgétaire, responsable de la diffusion, de l'information et de la formation.

114. L'une des conséquences de la LOLF a été l'apparition de nouveaux acteurs dans le paysage administratif français. Par exemple, le directeur du programme a dû trouver et se faire sa place entre le directeur des finances et le directeur de la GRH. De plus, les préfets jouaient un nouveau rôle en tant que coordinateurs de l'action publique chargés de veiller à la cohérence au niveau vertical comme au niveau horizontal au sein de la structure administrative. Ces nouveaux acteurs assumaient différents rôles et leur premier défi a été de trouver leur légitimité et leur organisation propre au sein de l'organisation. La mise en œuvre de la LOLF a également introduit de nouveaux thèmes dans le service public tels que le contrôle de la performance et de la qualité. II a fallu modifier l'inspection, qui est passée d'un contrôle ex-ante à un contrôle ex-post.

115. La stratégie d'information et de communication consistait en l'organisation de colloques destinés à expliquer la réforme. Le premier colloque s'est déroulé en octobre 2001 à l'occasion du lancement de la LOLF. Début 2002 le plan de communication a été lancé ; il consistait à travailler avec les médias, les groupes influents, les autorités locales et le Parlement. Internet a été un outil utile pour atteindre un public plus large, car il a permis, à l'aide de lettres d'information publiques, d'expliquer les nouveaux développements sur le plan de la gestion du budget et de ses implications pour la société. Plusieurs documents pédagogiques ont été publiés et de bonnes pratiques en matière de gestion du budget et d'expérimentation ont été largement diffusées.

116. Les travaux préparatoires on notamment consisté à tester les nouveaux concepts et les nouvelles procédures envisagées par la LOLF. Pour la France cela a également représenté une nouvelle

23 La LOLF est le fruit d'une initiative parlementaire proposée par le député Didier Migaud et le sénateur Alain Lambert avec l'approbation du président de l'Assemblée nationale, Laurent Fabius.

24 C'est ce qui est connu sous le nom de « performance à la française ». 
étape dans la mise en œuvre de l'action publique. II s'agissait de tester les nouveaux concepts et les nouvelles procédures pour voir comment elles fonctionnaient ensemble. Plusieurs organisations se sont portées volontaires pour tester les nouveaux concepts, procédures et outils budgétaires. Les organisations procédant aux tests se sont réunies un certain nombre de fois pour examiner les aspects les plus complexes de la LOLF, en particulier les relations entre les objectifs et les indicateurs. Ces discussions ont permis à chacun d'apprendre des autres, en particulier au sujet de la performance. ${ }^{25}$ La coordination des tests a été confiée à la Direction de la réforme budgétaire (DRB) au sein du ministère de l'Économie et des Finances.

117. La formation a constitué un autre aspect capital des travaux préparatoires avant l'exécution de la LOLF. Le processus de formation a été divisé en deux étapes. La première avait pour objectif la sensibilisation à la nouvelle réglementation. Le rôle d' " ambassadeurs » de la LOLF créé exigeait une formation spéciale ${ }^{26}$. L'École de la LOLF a été créée dans le cadre de la structuration de l'offre de formation. La deuxième étape a consisté à créer des communautés de pratiques dans le but de forger une doctrine, la formation étant plus axée sur la formation des directeurs de programme, des personnes responsables de l'exécution du budget et des comptables. À titre d'exemple, en 2005 les autorités françaises ont publié un guide pratique sur les budgets de fonctionnement des programmes qui définissait une méthodologie pour le processus budgétaire et les relations entre les responsables des programmes et ceux du budget.

\section{La situation actuelle}

118. La LOLF est entrée pleinement en vigueur en 2006. La DRB, qui était chargée de l'étape de test, a été supprimée et la Direction du budget a repris les responsabilités de la gestion de la LOLF. Elle a été remplacée par la Direction générale de la modernisation de l'État créée au sein du ministère de l'Économie et des Finances. La mise en œuvre de la LOLF a constitué un défi pour les autorités françaises car elles ont dû faire évoluer une culture ancienne et profondément ancrée qui accordait bien plus d'importance aux procédures qu'aux résultats.

Figure 2 : La LOLF, un instrument de changement culturel

$1959-2001$

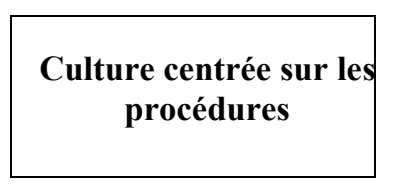

Source : OCDE (GOV)
$2001-2006$

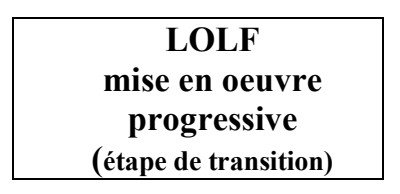

2006 -

Culture centrée sur les résultats (première loi de finances selon la LOLF)

119. On peut estimer que le nouveau mécanisme budgétaire est enfin intégré aux pratiques du service public. Toutefois, les relations entre les objectifs et les indicateurs de performance et leur pertinence sont encore en cours d'examen, et l'apparition de nouveaux acteurs exige l'élaboration dans certains ministères d'une charte définissant leur rôle.

120. La LOLF s'est également traduite par des changements importants dans le domaine de la gestion des ressources humaines (GRH). Le système de gestion a changé car il met maintenant l'accent

25 Les réunions étaient organisées par le ministère de l'Économie et des Finances et quelque 600 personnes y ont participé.

26 Il y avait environ 5 ou 6 « ambassadeurs » par ministère. 
sur les performances individuelles et insiste sur la nécessité de nouveaux outils pour gérer la masse salariale, d'un cadre conceptuel pour gérer les performances en $\mathrm{RH}$, et d'une restructuration des stages pour renforcer la formation.

121. Même si l'opinion des médias est favorable, les spécialistes ont des doutes quant à la mise en œuvre de la LOLF. Elle exigera d'autres changements tels que, par exemple, une nouvelle loi sur la fonction publique, une révision du système de comptabilité publique et un processus d'examen continu de la LOLF elle-même.

122. Globalement, la LOLF représente une révolution culturelle à l'intérieur du service public français. Elle s'est traduite par la redéfinition de certaines valeurs car elles sont désormais liées à la performance. II a fallu faire en sorte que chaque acteur ait le sentiment de participer pleinement au processus de réforme, en donnant une autre signification à leurs valeurs individuelles. Selon Françoise Waintrop, nous ne pouvons changer la culture sans changer les valeurs ${ }^{27}$. On estimait que le processus de modernisation devait avoir un sens pour l'ensemble des fonctionnaires pour qu'ils ne le considèrent pas comme une réforme purement technocratique. L'accent mis sur les résultats implique une évolution radicale des mentalités. Cela exige de nouvelles valeurs, attitudes, et priorités et la création d'un nouveau système de valeurs compatible avec une culture axée sur la performance.

123. La difficulté que rencontre à l'heure actuelle la LOLF est de passer de l'expérimentation à la généralisation. Cela s'est traduit par une sorte de période de stagnation. Lorsqu'elle fonctionnait sous forme de programme pilote dans certains domaines, la LOLF était considérée favorablement et la plupart des acteurs approuvaient le nouveau cadre conceptuel, mais au moment de la rendre applicable à tous, elle a suscité une résistance et certains se sont efforcés de la bloquer.

\section{Qu'est-ce qui a facilité la mise en œuvre de la réforme?}

124. La LOLF est une réponse aux inconvénients d'un cadre réglementaire daté qui n'a quasiment pas été modifié pendant toute sa période de validité et qui ne répondait pas au besoin d'une répartition plus équilibrée des pouvoirs. En effet, l'équilibre des pouvoirs entre l'exécutif et le législatif était inégal, du moins s'agissant de la gestion des finances publiques. De plus, plus de 30 tentatives de modification de l'ancien cadre réglementaire ont échoué. Ces éléments ont plaidé en faveur du besoin de réforme, en créant un sentiment d'urgence. Seule la combinaison de plusieurs facteurs, à savoir la vision d'avenir, le consensus politique, le leadership, le temps, les connaissances et un large débat, qui ont facilité la production du changement, a permis de faire évoluer la tradition déjà ancienne selon laquelle les procédures importaient plus que les résultats. De plus, l'objectif de la LOLF de faire un meilleur usage de l'argent public va clairement dans le sens des efforts pour réduire les dépenses publiques et faire des économies dans l'administration.

125. L'expérience de la LOLF montre combien il est important d'avoir une vision ou une idée claire de l'avenir souhaité et d'être en mesure de la faire connaître pour obtenir un soutien politique. Le fait qu'il s'agisse d'une proposition de réforme bipartisane et qu'elle soit approuvée par une majorité des membres de l'Assemblée nationale lui a apporté légalité et, plus important, légitimité. Ce que les réformateurs voulaient était clair: un meilleur équilibre des pouvoirs par le biais du contrôle du Parlement sur le budget et les finances publiques. Tous les acteurs publics étaient conscients de la nécessité de changement. Il est possible que les partis d'opposition, bien que conscients du besoin de réforme, aient soutenu l'initiative pour se servir du contrôle du Parlement sur le budget public comme

27 Françoise Waintrop, présentation lors de la réunion du Groupe de travail sur l'emploi et la gestion publics, 28 et 29 novembre, Paris. 
$d^{\prime}$ un instrument politique ${ }^{28}$. L'Assemblée nationale est le grand gagnant dans ce processus; après tout c'est une initiative en faveur du Parlement, puisqu'il obtient davantage de contrôle sur l'exécutif. Le bipartisanisme constitue dans ce cas une forme de coalition de cause dont le but est d'apporter un soutien politique à l'initiative.

126. Une action de réforme telle que la LOLF n'aurait pas été possible sans un leadership impliqué. Même si deux membres de l'Assemblée nationale peuvent être considérés comme les initiateurs visionnaires de la réforme, le leadership s'est matérialisé dans le pouvoir législatif lui-même. Cette implication des gestionnaires de moyen et de haut niveau a permis de renforcer le leadership. Si l'opposition politique était minime, la résistance ne pouvait venir que des acteurs chargés de la mise en œuvre et de l'exécution du nouveau processus budgétaire, susceptibles de considérer les nouvelles réglementations comme trop compliquées, perturbant leurs procédures de fonctionnement traditionnelles et engendrant une incertitude sur le plan de leurs conditions de travail. Une campagne d'information dans les médias et l'organisation de colloques a permis d'expliquer la réforme et de limiter la résistance au changement.

127. Les travaux préparatoires de la LOLF illustrent combien il est opportun de mettre en œuvre progressivement une action de réforme afin que les parties prenantes saisissent ses aspects conceptuels et techniques. Dans le même temps, cela favorise un processus d'adaptation à une nouvelle culture, à de nouvelles valeurs et à un nouvel environnement et de nouvelles pratiques de travail. II a fallu cinq ans aux autorités françaises pour expliquer la réforme, former le personnel, la faire connaître et informer les fonctionnaires et la société des changements et de leur nécessité avant que la première loi de finances sur le modèle de la LOLF ne soit enfin mise en œuvre. Le partage d'informations et la diffusion de connaissances ont été les facteurs essentiels, dans une large mesure, d'une transition sans heurts vers la pleine mise en oeuvre de la LOLF. La formation des gestionnaires était un élément déterminant non seulement pour expliquer les aspects techniques du processus budgétaire mais aussi pour créer un sentiment d'adhésion à la réforme et aux nouvelles idées ellesmêmes.

128. Les essais pilote menés dans le cadre de l'action représentaient une innovation dans le processus d'élaboration des politiques français et constituaient un élément fondamental de la gestion du changement car ils ont favorisé une adaptation et une compréhension progressive de la réforme. L'expérimentation est possible lorsque les actions de réforme sont mises en œuvre progressivement ; elle doit s'accompagner d'un vaste débat sur les résultats et d'un échange d'expériences et d'informations. En effet, les autorités financières françaises ne disposaient pas du cadre conceptuel global nécessaire à la mise en œuvre la LOLF ; il a fallu un énorme travail intellectuel pour mettre au point la base conceptuelle de la réforme en fonction du contexte local. ${ }^{29}$ Les concepts et procédures étaient nouveaux et il a fallu les tester avant de généraliser leur application. II faut également tenir compte du fait que l'expérimentation encourage le débat et l'apprentissage et l'amélioration de la réforme.

\section{En conclusion - quels sont les enseignements pour l'avenir ?}

129. À ce stade on peut estimer que le changement a été intégré aux procédures budgétaires mais il faudra encore du temps pour qu'il fasse partie intégrante de la culture du service public et des mentalités. On peut tirer au moins sept enseignements en matière de gestion du changement dans

28 Cet argument exige encore des analyses et des éléments d'appréciation empiriques supplémentaires.

29 C'est la raison pour laquelle les responsables français de l'élaboration des politiques parlent de concepts tels que la «performance française ». 
l'administration de la mise en œuvre de la LOLF. Ils ont été énumérés tout au long de l'étude de cas mais on peut les résumer de la manière suivante :

- La nécessité de former une vision d'avenir et de mettre au point une stratégie pour y parvenir.

- Le besoin d'un soutien politique et la constitution d'une coalition directrice pour diriger l'effort de changement.

- Le besoin d'un leadership fort et impliqué ayant suffisamment d'autorité morale et juridique pour encourager les membres de la coalition à travailler ensemble comme une équipe.

- Il est opportun de mettre en œuvre progressivement la réforme car cela facilite la compréhension, l'adaptation et la formation d'un sentiment d'appartenance. Il faut un lapse de temps suffisant adapter les mentalités à un nouvel environnement.

- L'utilisation de différents canaux de communication pour expliquer la réforme et partager les informations, par exemple : les médias, les colloques, les groupes influents et Internet.

- Le recours à la formation pour éliminer les obstacles à la réforme et l'expliquer de manière plus approfondie.

- Il est opportun de tester l'action envisagée pour encourager la formation d'un système d'apprentissage par le partage d'expériences et l'amélioration de l'action de réforme. 


\section{ITALIE - LE CAS DE LA RÉFORME DE LA FONCTION PUBLIQUE}

\section{Introduction}

130. Le service public italien connaît un processus continu de transformation depuis plus d'une décennie. Les contraintes économiques et l'influence des pairs ont entraîné des adaptations dans la gestion de la fonction publique. Cependant, les nouvelles réformes se sont heurtées à une résistance sur les fronts politique, culturel et comportemental ce qui a compromis la consolidation des efforts de réforme. L'expérience italienne montre combien il est utile d'obtenir un soutien politique pour produire et gérer le changement dans le service public mais aussi la nécessité de conserver ce soutien politique tout au long du processus pour atteindre les objectifs de réforme. Le but de cette étude de cas est d'analyser la manière dont les autorités italiennes gèrent le changement et font face à l'opposition aux initiatives de réforme.

\section{Quel est l'objet de la réforme?}

131. La première étape de la réforme du service public italien a commencé au début des années 90 et a été considérée comme achevée en 1998. La deuxième étape correspond à un processus de consolidation et à l'amélioration des mesures de réforme en fonction de priorités politiques, économiques et sociales; ce processus, en revanche, n'est pas terminé, de nombreux objectifs de la réforme n'ayant pas été atteints, tels que la mise en place d'un système efficace d'évaluation des services et de la satisfaction des usagers, la normalisation des pratiques de gestion des ressources humaines $(G R H)$, une plus grande efficacité en matière de $G R H$, et de nouvelles réductions des dépenses de personnel.

132. Les objectifs généraux ont consisté à faire en sorte que l'administration publique soit proche des citoyens, que l'efficience et l'efficacité de la prestation des services publics augmentent, et que les dépenses de personnel diminuent. Dans cet ordre d'idées, certaines des mesures adoptées sont les suivantes : i) un processus de modernisation des structures, fonctions et procédures de l'organisation ; ii) la prestation de services dans une optique de ciblage; iii) une redéfinition de la mission des organismes publics; iv) une amélioration qualitative des services publics; et, v) les attributions des gestionnaires en matière de gestion des ressources humaines. Globalement, cela a représenté un changement culturel à l'intérieur de l'administration publique italienne.

133. La fonction publique a été l'un des domaines de réforme de ces dernières années. La fonction publique italienne a connu des changements au titre d'une optique de gestion publique. La gestion des fonctionnaires au moyen de règles et d'évaluations se rapproche de plus en plus des pratiques du secteur privé. Afin de limiter l'influence politique dans la fonction publique, les nouvelles mesures prévoient une différenciation des rôles des fonctions politiques et managériales. De plus, la mise en place d'un processus de négociation collective avec les représentants des syndicats, l'instauration d'un processus d'évaluation des performances et l'adoption d'une sorte de système de rémunération liée aux performances sont certaines des mesures destinées à consolider le processus global de réforme, en transformant la culture administrative traditionnelle de la fonction publique. 


\section{Quels sont les moteurs de la réforme ?}

134. Des facteurs structurels internes et externes sont à l'origine des réformes dans la fonction publique italienne. Des contraintes budgétaires et une gestion peu efficace des fonds publics sont les deux principaux facteurs internes qui ont déclenché la réforme. L'influence des autres pays de l'Union européenne (UE) et l'exploitation délibérée des enseignements tirés d'autres pays constituent les principaux éléments extérieurs qui ont facilité le processus de réforme.

135. Selon le Département de la fonction publique (dont les initiales italiennes sont DFP) ${ }^{30}$ il existe une forte corrélation entre le niveau de compétitivité du pays et la performance de l'administration publique. Partant, il juge nécessaire d'améliorer la qualité des politiques publiques et de la prestation de services pour appuyer les efforts destinés à renforcer la compétitivité économique et la croissance du pays. La nécessité de faire des économies dans les dépenses publiques a conduit à répartir différemment les ressources humaines entre les agences et ministères ainsi qu'entre les différents niveaux de l'administration.

136. L'expérience des pays Membres de l'UE a également suscité des réformes à l'intérieur du service public. En fait, le système d'examen par les pairs est considéré comme l'un des instruments de promotion de l'autoévaluation par l'administration italienne. En d'autres termes, les processus d'apprentissage en matière d'action publique ou de transfert délibéré d'actions ont entraîné des changements dans le service public, ce qui a ouvert des possibilités d'amélioration, d'actualisation et a renforcé la consolidation du processus de réforme.

\section{Qu'est-ce qui a changé ?}

137. Les réformes mises en œuvre il y a plus de dix ans ont modifié la culture administrative dans le service public italien. Le changement se manifeste dans les conditions de travail des fonctionnaires, l'introduction d'une gestion orientée vers les performances, et une administration orientée vers le service. Les pratiques traditionnelles et les particularités italiennes ont été radicalement modifiées pour rendre le service public plus réceptif et fiable. Cependant, ce processus de changement se poursuit et exige une consolidation ou une adaptation constantes des programmes de réforme. En effet, aujourd'hui les fonctionnaires doivent rendre des comptes aux citoyens en cas de retards dans les services administratifs, mais leurs résultats importent moins en raison de l'absence d'un système d'évaluation. La figure 2 illustre le processus continu de changement qu'a connu la culture du secteur public italien au cours des dix dernières années.

138. Bien qu'il reste encore beaucoup de chemin à parcourir pour consolider les réformes entreprises dans les années 90 , certains progrès ont été réalisés dans le sens où la manière de fonctionner dans le service public s'est transformée. Pour consolider les réformes et éviter un retour en arrière, l'État italien a remanié ses programmes et procédures pour améliorer et renforcer le processus de réforme. L'importance de l'autoévaluation (autovalutazione) à l'aide du Cadre d'autoévaluation des fonctions publiques (CAF) de l'UE, de l'évaluation par les pairs et de l'évaluation comparative est de plus en plus reconnue. L'objectif est d'instaurer une culture d'amélioration et d'apprentissage continus à l'intérieur du service public qui permette d'offrir des services publics de meilleure qualité.

30 Dipartimento de la Funzione Pubblica (DFP). 
Figure 3. Évolution du service public italien dans le cadre d'une approche managériale

Service public avant les années 90

\footnotetext{
- Hautement centralisé

- Fortement influencé par les événements politiques
}

- Clientélisme

- Fonctionnaires influencés par le favoritisme politique

- Inefficacité en matière de gestion des ressources

- Réceptivité, transparence et obligation de rendre des comptes limitées

- Mobilité limitée

\begin{tabular}{|c|c|c|}
\hline $\begin{array}{c}\text { Service public } 15 \text { ans } \\
\text { plus tard }\end{array}$ & Défis restant à relever & Effets att \\
\hline Étape o & Consolidation & $\begin{array}{c}\text { Culture } \\
\text { d'amélioration } \\
\text { continue }\end{array}$ \\
\hline $\begin{array}{l}\text { - Plus de souplesse en } \\
\text { matière de GRH }\end{array}$ & $\begin{array}{l}\text { - Un proc } \\
\text { des polit }\end{array}$ & \\
\hline $\begin{array}{l}\text { - Orienté vers les usagers } \\
\text { - Service public plus } \\
\text { décentralisé }\end{array}$ & $\begin{array}{l}\text { - Séparation entre le } \\
\text { politique et la gestion } \\
\text { - Davantage de transparencє }\end{array}$ & $\begin{array}{l}\text { Satisfaction des } \\
\text { besoins de la } \\
\text { population }\end{array}$ \\
\hline $\begin{array}{l}\text { Efficacité améliorée en } \\
\text { matière de gestion des } \\
\text { ressources }\end{array}$ & $\begin{array}{l}\text { Obligation de rendre des } \\
\text { comptes et réceptivité } \\
\text { accrues }\end{array}$ & $\begin{array}{l}\text { Restauration de } \\
\text { la confiance de }\end{array}$ \\
\hline $\begin{array}{l}\text { - Prestation des services } \\
\text { publics orientée vers la } \\
\text { qualité }\end{array}$ & $\begin{array}{l}\text { - Amélioration de la qualité } \\
\text { de la prestation des } \\
\text { services publics à tous les } \\
\text { niveaux de l'administratior }\end{array}$ & $\begin{array}{l}\text { la population à } \\
\text { l'égard de } \\
\text { l'administration } \\
\text { publique }\end{array}$ \\
\hline & 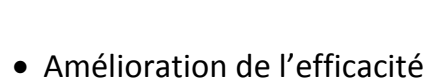 & \\
\hline $\begin{array}{l}\text { Différenciation des } \\
\text { fonctions politiques et } \\
\text { managériales }\end{array}$ & $\begin{array}{l}\text { et de l'effectivité en } \\
\text { matière de GRH }\end{array}$ & Légitimité accrue \\
\hline
\end{tabular}
dans le cadre d'une approche managériale

\section{Mise en œuvre de réformes}

Source : OCDE (GOV)

\section{Comment le changement est-il géré ?}

139. Les changements intervenus dans le service public se sont heurtés à une résistance politique, culturelle et comportementale. Les hommes politiques et les représentants des syndicats ont eu le sentiment que leurs intérêts étaient affectés par la mobilité croissante ou la répartition des fonctionnaires entre les différents secteurs et/ou niveaux de l'administration et se sont donc opposés aux réformes. La résistance culturelle s'est exprimée dans la notion selon laquelle l'administration publique a un rôle social à jouer dans la répartition des ressources économiques. De plus, l'idée de modifier des habitudes et pratiques déjà anciennes a suscité une résistance comportementale de la part des gestionnaires, des fonctionnaires et des dirigeants syndicaux. Partant, la culture traditionnelle du service public a également constitué un obstacle au changement. Les pratiques institutionnalisées et les mentalités se sont avérées difficiles à changer, particulièrement en raison d'une fonction publique vieillissante. L'ouverture et la réceptivité des individus aux nouvelles idées se sont révélées relativement limitées.

140. Pour affronter la résistance, les pouvoirs publics italiens ont pris deux mesures: la participation de la société civile et de l'ensemble des parties prenantes et l'utilisation des médias comme canal de communication pour expliquer les réformes. Le dialogue et le partage d'informations ont fortement contribué à surmonter la résistance aux réformes, l'ensemble des parties prenantes et des autres acteurs concernés ayant besoin de comprendre ce qui change et pour quelle raison. Les médias, par exemple, ont joué un rôle décisif en rendant plus transparents les processus de prise de 
décision, les dépenses et nominations de personnel. De plus, la formation continue a joué un rôle déterminant dans la familiarisation des gestionnaires avec les aspects conceptuels et techniques de la réforme. Le problème, cependant, c'est que la formation continue est parfois moins importante que l'ancienneté pour l'avancement professionnel, ce qui n'incite donc pas les individus à participer aux sessions de formation.

141. Comme indiqué plus haut, la réforme de la fonction publique italienne est en cours depuis plus de dix ans. La mise en œuvre progressive et par paliers des réformes devrait favoriser peu à peu la réceptivité et une meilleure adaptation des individus et des institutions à de nouvelles idées. Cependant, peu de progrès ont été réalisés en Italie où une population vieillissante et la persistance d'anciennes pratiques gênent le développement de nouvelles méthodes managériales et l'institutionnalisation d'une culture d'amélioration continue.

\section{En conclusion - quels sont les enseignements pour l'avenir?}

142. L'expérience italienne montre que pour qu'une réforme réussisse il est nécessaire d'obtenir un soutien politique mais dans le même temps d'éviter d'être influencé par des intérêts politiques ou élitistes. Certains acteurs politiques peuvent voir leurs intérêts et leur influence affectés par leur réforme et peuvent par conséquent s'efforcer de modifier l'orientation de la réforme. De ce fait, la gestion du changement requiert non seulement un soutien politique mais aussi le maintien de ce soutien par des processus de négociation dirigés par un leadership de confiance. Les dirigeants doivent veiller à empêcher les forces politiques de peser sur le processus de réforme et de lui donner une orientation qui favorise uniquement certains groupes au sein de l'arène politique.

143. On peut tirer au moins trois autres enseignements apparentés en matière de gestion du changement. Les examens par les pairs et l'évaluation comparative constituent, dans une certaine mesure, des moteurs extérieurs du changement. Le fait d'apprendre des expériences des autres pays favorise des processus libres de transfert et, en conséquence, engendre des forces structurelles favorables à la production du changement. Les autorités italiennes ont reconnu le rôle important que jouent ces instruments de réforme pour l'obtention d'idées et d'enseignements dans une optique d'amélioration continue.

144. Par ailleurs, la réforme italienne montre qu'il faut du temps et un engagement politique pour que le changement se produise. Enfin, la réforme de la fonction publique italienne démontre clairement que la gestion du changement exige une part d'auto-critique. En d'autres termes, les gouvernements doivent développer leur capacité d'autoévaluation et déterminer ce qu'il faut améliorer pour consolider le processus de réforme. Ainsi, il est nécessaire d'être ouvert à l'évaluation externe et interne pour améliorer les processus de réforme en cours. Qui plus est, le changement peut être considéré comme une activité d'expérimentation continue dont on peut tirer des enseignements positifs et négatifs. 


\section{PORTUGAL - LE CAS DU PROCESSUS DE RÉFORME DE L'ADMINISTRATION PUBLIQUE}

\section{Introduction}

145. Au cours des dernières décennies les autorités portugaises ont pris des mesures de réforme spécifiques au service public. Cependant, un processus de modernisation intégré visant à garantir l'efficacité de l'administration publique et à appuyer la stratégie de croissance économique n'a commencé qu'au deuxième semestre 2005. Les mesures adoptées, en dépit d'une certaine résistance de la part des fonctionnaires, sont néanmoins jugées nécessaires par les agents économiques et la société civile. Les médias, les membres de la société civile et les acteurs économiques ont exercé des pressions considérables sur les pouvoirs publics pour qu'ils accélèrent la réforme. La présente étude de cas vise à analyser la manière dont les autorités portugaises intègrent le changement dans la culture du secteur public et affrontent la résistance aux mesures de réforme. Certains enseignements importants en matière de gestion du changement peuvent être tirés de l'expérience portugaise.

\section{Quel est l'objet de la réforme?}

146. En 2005, le gouvernement portugais nouvellement élu a pris les premières mesures de remaniement de la stratégie de modernisation de l'administration publique. Il a fait de la réforme de l'administration publique une priorité et a introduit de grands changements dans le régime juridique des fonctions publiques. Son but était d'instaurer progressivement dans le secteur public une nouvelle culture de la gestion par objectifs, ainsi qu'une culture de l'évaluation et de l'obligation de rendre des comptes qui crée de meilleures relations entre l'État et les citoyens, facilite la prestation des services publics et favorise le développement économique.

147. En ce sens, la réforme de l'administration publique portugaise repose sur cinq piliers : i) la restructuration de l'administration centrale de l'État (PRACE) ; ii) la mobilité ; iii) le remaniement du système de carrière et de rémunération; iv) le système d'évaluation des performances; et v) la simplification administrative/l'administration en ligne.

148. Le programme de restructuration de l'administration centrale de l'État (PRACE) qui a commencé au deuxième semestre 2005 a pour ambition de favoriser le développement économique et d'améliorer la qualité des services publics. Il cherche à réduire le nombre de services publics de l'administration directe et les ressources qui leur sont affectées, à savoir le nombre de fonctionnaires qui travaillent dans ces services publics. Globalement, son but est de réduire les dépenses, de décentraliser les fonctions, de coordonner la déconcentration et de moderniser et automatiser les procédures, ainsi que d'accroître l'efficacité par des processus de gestion et de coordination plus performants.

149. Le système de mobilité des ressources humaines $(S M E)$ vise une meilleure utilisation et une meilleure répartition des ressources humaines en redéfinissant les structures et en introduisant un nouveau cadre pour la mobilité. L'objectif est de redéfinir les besoins en effectifs en fonction des nouveaux services gouvernementaux. À cet effet, les hauts responsables ont été habilités à évaluer et adapter la structure administrative existante et les besoins en personnel. 
150. La réforme de l'administration publique envisage le remaniement du système de carrière et de rémunération actuel. Le remaniement du système de carrière est principalement dû aux multiples types de statut juridique et au nombre excessif de carrières qui le composent, au manque de transparence de son système de rémunération et à l'extrême importance de l'ancienneté pour les promotions. Les réformes envisagent l'adoption de deux instruments juridiques uniquement: un contrat de travail pour les fonctions publiques et un statut spécial (nomination) pour les fonctions liées à la souveraineté (affaires étrangères, forces armées, sécurité, justice et inspection). De plus, les nouvelles mesures réduisent les carrières au type général et spécial, adoptent un barème de rémunération, et favorisent la souplesse dans le recrutement et la détermination des salaires. Le nouveau système de carrière et de rémunération entrera en vigueur début 2008.

151. Dans le même temps, le nouveau système intégré d'évaluation des performances dans l'administration publique (SIADAP) est entré en vigueur fin 2007. C'est l'un des grands atouts de la stratégie de modernisation de l'administration publique. Le système précédent mettait uniquement l'accent sur l'évaluation des cadres moyens et des employés et a été critiqué car il était jugé complexe et bureaucratique. Les nouvelles mesures incluent l'évaluation des performances des services, des cadres moyens et supérieurs et des employés. Ces mesures visent à créer un environnement de compétition, car les résultats de l'évaluation des performances auront un impact sur les rémunérations du personnel et sur l'avancement professionnel ainsi que sur le renouvellement des contrats. Le nouveau SIADAP vise à développer, d'une manière cohérente et intégrée, un système d'évaluation global pour améliorer la motivation professionnelle et les performances de l'administration publique.

152. En ce qui concerne la modernisation et la simplification administrative, le programme pour une meilleure législation vise à améliorer la qualité des lois votées par les pouvoirs publics, à rendre l'État moins bureaucratique et à faciliter la vie des citoyens et des entreprises. Parallèlement à la simplification administrative, l'administration électronique est aussi une étape décisive dans la recherche d'une compétitivité, $d$ 'une croissance économique et d'une qualité de vie supérieures. En ce sens, le programme "Simplex " a été conçu pour réduire la bureaucratie, accroître la transparence de l'État et favoriser l'efficacité dans l'administration publique.

\section{Quels sont les moteurs de la réforme?}

153. Le changement dans le secteur public portugais a été déclenché principalement par des facteurs internes : des contraintes budgétaires, le besoin d'accroître l'efficacité et la mauvaise image du service public. Cela a conduit les pouvoirs publics portugais à rechercher d'autres solutions pour réduire les dépenses sans pour autant oublier leurs objectifs en matière de politique sociale. Pour ce faire, le Portugal a notamment procédé à une réforme du service public sur le plan de la réglementation, des procédures et de la gestion des ressources humaines.

154. L'opinion publique voyait le secteur public comme un organisme extrêmement surdimensionné et comme une entrave au développement économique. Cette situation suscitait des interrogations quant à l'efficience et l'efficacité de la performance de l'administration et diminuait la confiance des citoyens à l'égard du service public, ce qui mettait en péril la légitimité de l'État et ses efforts de réforme sur les fronts économique et social. Cette perception négative se manifestait clairement dans les résultats de certaines études et dans le nombre de revendications des citoyens, en particulier des hommes d'affaire et des associations professionnelles.

155. Partant, pour restaurer l'opinion favorable des citoyens à l'égard de l'administration publique et atteindre leurs objectifs budgétaires, les autorités portugaises ont adopté des mesures pour 
renforcer la stratégie de modernisation de l'administration publique afin de la rendre plus rentable, transparente et responsable.

\section{Qu'est-ce qui a changé ?}

156. Le vaste ensemble de réformes a entraîné une transformation culturelle du service public portugais depuis $2004^{31}$. La gestion par objectifs définie dans la réforme a constitué une formidable matrice du changement. La mise en œuvre de la gestion par objectifs a introduit une certaine rigueur méthodologique dans l'évaluation des ministères, des organisations et des unités organiques et dans l'évaluation des performances des individus. L'élargissement de la portée de l'évaluation des performances aux services, aux cadres supérieurs et moyens et au personnel de niveau inférieur, et son rattachement aux rémunérations et à l'avancement professionnel représente également un grand changement dans la culture administrative portugaise. Ces mesures visent à institutionnaliser un modèle d'évaluation fiable qui est indispensable à l'introduction d'une culture de la gestion des performances.

157. Les changements intervenus dans la culture administrative ont favorisé l'apparition de nouvelles valeurs dans le service public. L'obligation de rendre des comptes, l'ouverture, la transparence, la flexibilité, le leadership et une méthode managériale sont autant d'éléments qui feront bientôt partie intégrante de la nouvelle culture administrative. Les notions de mesure des performances, de fixation d'objectifs, d'obligation de rendre des comptes, de responsabilité et d'autonomie accrue pour les cadres supérieurs et moyens, le fait de lier l'avancement professionnel aux performances et aux disponibilités budgétaires commencent à être assimilé dans le service public, non sans une certaine résistance cependant.

158. Les autorités portugaises ont entrepris un processus de réforme progressif et intégré. Les réformes sont conduites par le secrétaire d'État à l'administration publique, avec le soutien du Premier ministre. Afin de faciliter la compréhension des réformes, la direction générale de l'administration et de la fonction publique (DGAEP) organise des séminaires pour l'ensemble de l'administration publique ainsi que des sessions d'information dans les services qui le demandent et apporte une aide technique aux services publics en définissant des orientations techniques pour faciliter l'interprétation des mesures de réforme. L'institut national d'administration (INA), un organisme de formation, a organisé des stages de formation professionnelle sur des questions spécifiques de réforme telles que: l'évaluation des performances, le contrat de travail individuel, le système de mobilité et le nouveau régime juridique de la fonction publique.

\section{Comment le changement est-il géré ?}

159. La mise en œuvre de ces réformes a incontestablement entraîné des changements dans la culture traditionnelle du service public portugais. Néanmoins, les mesures ne suscitent pas l'enthousiasme des fonctionnaires car ils ont le sentiment que certaines réformes affectent leurs conditions de travail et les droits des travailleurs. À titre d'exemple, dans le cadre des efforts de réduction des dépenses publiques, le régime de retraite des fonctionnaires a été transformé pour le

31 L'administration publique portugaise a connu un processus continu de réforme mais la méthode a évolué avec le temps en fonction des priorités du gouvernement du moment. À la fin des années 80 et au début des années 90 , les efforts de réforme visaient principalement la restructuration du système de rémunération et la clarification du système de carrière et du statut juridique des fonctionnaires ainsi que l'amélioration du système de mobilité. À partir de la deuxième moitié des années 90 jusqu'en 2000-2002, la tendance a évolué avec la création d'un grand nombre de services, la règle étant le recours à des contrats de travail et une autonomie accrue. Les contraintes budgétaires ont rendu nécessaire un contrôle accu des recrutements de personnel. 
rapprocher du régime de sécurité sociale général qui est moins favorable. De plus, d'aucuns ont le sentiment que les efforts de réforme actuels dans le secteur public donnent davantage de pouvoir aux gestionnaires (trop du point de vue des employés), qui joueront un rôle plus décisif en matière d'avancement professionnel. Les médias et les acteurs économiques sont favorables aux réformes, qu'ils jugent nécessaires, et ils ont donc contribué à faciliter le changement ${ }^{32}$. Les médias se sont fréquemment fait l'écho des réformes de l'administration publique, poussant généralement les pouvoirs publics à accélérer le processus de réforme.

160. Les cadres supérieurs et moyens sont responsables de la mise en œuvre des mesures de réformes. Cependant, ils n'ont pas toujours les compétences nécessaires pour assumer le rôle de dirigeants et contribuer à la production et à la gestion du changement. En conséquence, la formation à l'aide de stages et de séminaires spécialisés a pour but d'apporter de nouvelles connaissances et de nouvelles compétences (comme le leadership), de motiver le personnel et de faciliter l'intériorisation du changement. De plus, la formation professionnelle est désormais obligatoire pour les cadres. Il existe également des cours de perfectionnement pour les nouveaux cadres et les cadres en poste. En somme, la formation professionnelle fait partie intégrante de la stratégie de réforme de l'administration publique qui joue un rôle décisif dans la gestion du changement et, dans une large mesure, dans la réussite des programmes de réforme.

\section{En conclusion - quels sont les enseignements pour l'avenir?}

161. On peut tirer des enseignements en matière de gestion du changement de l'expérience portugaise de la réforme du service public. D'une part, il est nécessaire de maintenir la dynamique et l'efficacité des réformes pour tirer profit des mesures qui ont déjà été prises par les gouvernements précédents, lesquelles étaient jugées essentielles et importantes, en les adaptant aux nouvelles priorités politiques. Cela a été le cas, par exemple, de l'organisation administrative, de la révision du statut des gestionnaires et de l'évaluation des performances. Cela évite de gaspiller des ressources et de perdre du temps. Des facteurs internes tels que le déficit budgétaire et la nécessité d'accroître l'efficacité des services publics sont à l'origine de changements dans les programmes de réforme.

162. D'autre part, l'expérience portugaise montre que pour intégrer réellement le changement à la culture du secteur public, il est judicieux de le faire progressivement, parallèlement à une stratégie de communication et d'information efficace, qui permet à toutes les parties prenantes de comprendre ce qui a changé et pour quelle raison. Le but est de favoriser un climat d'adhésion et d'adaptation des mentalités, de la culture et des attitudes du personnel du service public à un nouvel environnement et à de nouvelles conditions de travail.

163. Une réforme intégrée de l'administration publique compatible avec d'autres réformes de l'action, qui a remplacé les mesures de réforme spécifiques mises en œuvre au cours des quatre dernières années, s'est révélée plus rationnelle et productive en raison du fort impact qu'elle a eu sur le système de l'administration publique. Elle s'avère également plus cohérente et compréhensible pour les citoyens.

164. Il a également été estimé qu'un leadership impliqué est un facteur indispensable à la réussite des réformes et à la gestion du changement. C'est pourquoi la consolidation des compétences des gestionnaires a fait l'objet d'autant d'investissements, en particulier en ce qui concerne la direction, la motivation et de nouvelles méthodologies de travail. II faut également du temps pour que les

32 Des associations professionnelles telles que «Compromisso Portugal» ont joué un rôle important dans la définition du besoin de changement. 
changements s'installent, en particulier ceux qui impliquent l'adoption de nouvelles valeurs et d'une nouvelle culture, car ceux-là ne se font pas par décret. II faut permettre aux acteurs concernés de comprendre le besoin de réforme et leur donner du temps pour s'adapter à de nouvelles conditions de travail. L'adaptation ne signifie cependant pas le report de l'introduction de la réforme. Une mise en œuvre progressive de la réforme permet l'adaptation des mentalités, des attitudes et du comportement des individus à un nouvel environnement tout en mettant en route le mouvement favorable à la concrétisation du changement. 


\section{ESPAGNE - LE CAS DU STATUT DE BASE DE L’EMPLOYÉ PUBLIC}

\section{Introduction}

165. L'Espagne entreprend actuellement un processus de réforme de grande ampleur de la gestion des ressources humaines dans le secteur public. Le Statut basique de l'employé public est supposé donner de la cohérence à des pratiques variées de gestion des ressources humaines aux trois niveaux de l'administration. II introduit des techniques s'inspirant du secteur privé pour moderniser la culture administrative traditionnelle. Cependant, bien qu'elle n'en soit qu'à ses balbutiements, cette transformation culturelle présente des difficultés, la méconnaissance des nouvelles pratiques ayant suscité des doutes dans le service public. L'objectif de la présente étude de cas est d'analyser la manière dont les autorités espagnoles intègrent le changement dans la culture du service public et tire des enseignements en matière de gestion du changement.

\section{Quel est l'objet de la réforme?}

166. Le Statut basique de l'employé public (dont les initiales espagnoles sont EBEP) ${ }^{33}$ a pris effet le 13 mai 2007 et constitue l'une des grandes réformes de la gestion des ressources humaines (GRH) dans le secteur public espagnol de ces dernières années. II s'agit d'un dispositif de gestion unique applicable aux 2,5 millions de fonctionnaires et employés publics de l'administration centrale, des régions autonomes (appelées "communautés autonomes") et des entités locales. II vise à améliorer les conditions de travail des employés publics et, dans le même temps, la qualité des services publics en instaurant une culture orientée vers les performances.

167. Les partis politiques, les communautés autonomes, les entités locales, les agents sociaux, les syndicats et les citoyens ont participé aux débats dans le cadre de rencontres coordonnées par le Ministère des administrations publiques (dont les initiales espagnoles sont MAP) afin d'enrichir le premier projet de l'EBEP. Le débat parlementaire a débouché sur plus de 300 amendements au projet, qui a enfin été approuvé en avril 2007. À titre d'exemple, le champ d'application de l'EBEP est l'une des questions qui a suscité la plus grande polémique. II a été décidé, au terme d'un long débat, qu'il s'appliquerait à tous les membres du personnel travaillant pour l'administration publique : fonctionnaires et personnel temporaire.

\section{Quels sont les moteurs de la réforme?}

168. Ce sont des facteurs internes qui ont été les principales forces de déclenchement de la réforme du secteur public espagnol. Des vides juridiques, des pressions politiques et des processus administratifs datés sont les principaux facteurs qui ont conduit à la création de l'EBEP. Bien que la Constitution espagnole de 1978 exige la création d'un statut pour la fonction publique, celle-ci était encore régie par une loi préconstitutionnelle de 1964 sur la fonction publique, modifiée en partie par une loi de 1984. Par conséquent, l'EBEP vient combler un vide juridique dans la législation sur le service

33 Estatuto Básico del Empleado Público (EBEP). 
public et l'emploi public en créant un cadre normatif unique qui pose les principes de la gestion du service public.

169. Un nombre important de compétences ont été transférées aux communautés autonomes dans différents domaines depuis leur création par la Constitution de $1978 .{ }^{34} \mathrm{D}$ 'où la nécessité d'intégrer ces changements dans une seule loi-cadre de base. L'EBEP est maintenant la réglementation de base de la fonction publique et de l'emploi public, et devrait être complété par des lois et règlements centraux et régionaux. Si tout va bien cela devrait faciliter la mobilité et les possibilités d'avancement professionnel dans le service public espagnol aux trois niveaux de l'administration (central, régional et municipal).

170. Les gouvernements des communautés autonomes emploient plus de la moitié des agents publics du pays. Les municipalités locales ont également vu les effectifs de leur personnel augmenter considérablement ce qui s'est traduit par des différences dans les pratiques de GRH. Par ailleurs, une organisation bureaucratique traditionnellement fortement centralisée a été fragmentée par la création d'organismes de nature très différente qui a exigé réorganisation et coordination.

\section{Qu'est-ce qui a changé ?}

171. L'introduction de l'EBEP constitue une rupture importante dans la culture administrative traditionnelle espagnole, et ce pour trois raisons principales. Premièrement, il crée un modèle managérial prônant un minimum d'homogénéité dans la fonction publique qui s'applique à l'ensemble des employés du pays aux trois niveaux de l'administration. Deuxièmement, il instaure un système d'évaluation des performances au titre duquel les employés publics doivent rendre compte de leurs performances. ${ }^{35}$ Enfin, l'EBEP intègre un nouveau système de salaires lié en partie aux résultats de l'évaluation, qui se traduit par l'adoption d'une sorte de système de rémunération liée aux performances.

172. Les autres principaux changements incluent: a) une réglementation commune pour les fonctionnaires et les employés publics; b) une clarification des missions et des responsabilités des fonctionnaires et des employés publics; c) une souplesse accrue dans la détermination des salaires pour les régions et les municipalités; d) une nouvelle classification du personnel; e) un nouveau cadre pour les négociations collectives et les droits des syndicats; et, e) la nécessité d'une formation continue en fonction du contexte économique, social et technologique.

173. La mise en œuvre de l'EBEP s'est cependant heurtée à plusieurs complications, mais il est trop tôt pour pouvoir l'évaluer pleinement. L'intégration de la mobilité horizontale et verticale peut être source de conflits étant donné le manque traditionnel de mobilité dans la fonction publique. De plus, la conception d'un système unique d'évaluation des performances s'est avérée difficile, chaque unité indépendante présentant des caractéristiques administratives différentes.

\section{Comment le changement est-il introduit ?}

174. Trois principales lignes de travail visent à faciliter l'introduction progressive du changement dans le secteur public espagnol : la coordination entre les trois niveaux d'administration territoriale, la formation de gestionnaires publics et la mise à l'épreuve de plusieurs dispositions de la réforme avant sa pleine mise en œuvre.

34 Principalement les services de base tels que l'éducation, la santé et la dépendance future.

35 L'utilisation de la gestion des performances a été traitée en partie seulement par la loi de 1984. 
175. II existe plusieurs comités qui comprennent des représentants de l'administration centrale, des régions autonomes et des entités locales. L'administration centrale assure la coordination tout en respectant l'autonomie de chaque région. Ces comités travaillent à la mise au point de nouvelles orientations de l'EBEP portant sur l'amélioration du dialogue et de la coopération.

176. La mise en œuvre de l'EBEP exige la formation de ceux qui sont responsables de la gestion des ressources humaines aux différents niveaux de l'administration. L'objectif est de faciliter la compréhension des aspects techniques de la nouvelle réglementation tels que, entre autres: la classification du personnel, les règles de recrutement, l'évaluation des performances. Cependant, une formation plus structurée doit être adoptée ; en effet, pour le moment, les personnes qui ont participé à l'élaboration de l'EBEP rencontrent le personnel responsable de la GRH à tous les niveaux de l'administration pour répondre personnellement à leurs questions.

177. L'EBEP impose aux trois niveaux territoriaux de l'administration l'introduction de systèmes d'évaluation des performances. L'administration centrale pratique actuellement des tests d'évaluation des performances dans des délégations des régions autonomes. L'évaluation étant réalisée dans le cadre d'un programme pilote, ses résultats ne sont pas reliés aux salaires ni pris en compte pour les promotions pour le moment. L'expérience de ces tests pourra apporter des enseignements pour affiner l'action et les aspects techniques de l'évaluation mais ils permettront également une familiarisation et une adaptation progressive à la pratique, ce qui, de notoriété publique, est jugé déterminant pour la gestion du changement. De plus, il faut souligner que le système d'évaluation est perçu comme un surcroît de travail administratif dont l'efficacité est difficile à mesurer.

\section{Comment le changement est-il géré ?}

178. Même si l'application de l'EBEP n'en est qu'à ses débuts, il est possible de distinguer certains éléments de gestion du changement susceptibles d'ouvrir la voie au développement de l'action publique.

- L'implication politique et la participation sociale : l'approbation et l'introduction de l'EBEP a été facilitée par un processus ouvert d'élaboration des politiques. La participation d'un certain nombre d'acteurs politiques et de membres de la société civile aux débats sur le projet de réforme s'est révélée capitale pour obtenir la légitimité, un soutien et une implication politique de la part des autorités à chaque niveau de l'administration. Le besoin de changement a été ressenti à l'intérieur par les forces politiques et sociales à différents niveaux de l'administration, de sorte que l'ensemble des parties prenantes était acquis à la poursuite des mêmes objectifs. Selon le Ministère des administrations publiques (dont les initiales espagnoles sont MAP) ${ }^{36}$ le consensus se situait à trois niveaux: institutionnel (entre les communautés autonomes et la fédération espagnole des municipalités et des provinces), social (avec les syndicats), et politique (avec l'ensemble des partis politiques).

- Mise en œuvre progressive de la réforme : I'introduction de l'EBEP pourrait se traduire par un changement culturel dans le secteur public espagnol. La mise en œuvre progressive de la loi a pour but de faciliter l'adaptation et la familiarisation des fonctionnaires avec les changements. De plus, cela permettra d'éprouver les aspects techniques de la nouvelle législation dont on pourra tirer des enseignements positifs et négatifs.

36 Ministerio de las Administraciones Públicas (MAP). 
- Cohérence de l'action: l'EBEP est un cadre normatif général qui fait la synthèse des développements administratifs intervenus aux trois niveaux de l'administration. Ainsi, son acceptation est due, dans une certaine mesure, à sa compatibilité avec les pratiques locales.

- Formation des gestionnaires : elle contribue pour beaucoup à la compréhension de la réforme et à l'acceptation du changement.

\section{En conclusion - quels sont les enseignements pour l'avenir?}

179. Il y a au moins trois enseignements importants à tirer de la mise en œuvre de l'EBEP. Cela montre tout d'abord que la gestion du changement commence pendant le processus de formulation des politiques. Le dialogue avec différentes forces sociales et politiques et leur participation favorisent la création d'une conception d'avenir commune et l'engagement de l'ensemble des parties prenantes à la concrétiser. Cela limite indirectement le risque que la réforme ne soit perçue de façon négative et, en conséquence, l'apparition d'une résistance au changement.

180. Le deuxième enseignement est qu'il est opportun de mettre en œuvre progressivement l'action de réforme pour accroître la réceptivité au changement des institutions locales et des fonctionnaires. Le fait d'envisager le changement par paliers facilite l'adaptation à un nouvel environnement et la familiarisation avec les aspects conceptuels et techniques de la réforme. Ce sont deux éléments indispensables à une gestion efficace du changement.

181. Enfin, on peut souligner que, comme l'a montré la formulation et la mise en œuvre de l'EBEP, les politiques ne doivent pas seulement être compatibles avec des réformes plus générales mais avec des pratiques particulières à des niveaux inférieurs de l'administration lorsqu'ils sont directement touchés par les changements proposés. Cela contribue à l'acceptation et à la compréhension du changement.

182. La principale difficulté qui attend l'EBEP est l'institutionnalisation de la nouvelle culture administrative. Cela implique l'intégration complète d'aspects tels que la gestion des hauts fonctionnaires, l'évaluation des performances et la planification stratégique des $\mathrm{RH}$, ce qui exige une réglementation spécifique au niveau fédéral et dans les communautés autonomes. 


\section{LA SUISSE - LE CAS DE LA RÉFORME DU PERSONNEL}

\section{Introduction}

183. En 2005, le Conseil fédéral suisse a adopté une stratégie générale destinée à réformer le service public dont les structures et processus commençaient à devenir trop complexes et rigides et empêchaient les pouvoirs publics de répondre correctement aux besoins des citoyens. La stratégie vise à simplifier les procédures, à mieux structurer les processus et à éliminer les " double-emplois ". ${ }^{37}$ La réforme de l'administration fédérale fait partie d'un programme de réforme plus général visant à consolider le budget de la Confédération. En ce sens, la présente étude de cas a pour but d'analyser la manière dont les autorités suisses intègrent et gèrent le changement au sein du service public.

\section{Quel est l'objet de la réforme?}

184. En septembre 2005, le Conseil fédéral, conformément à la stratégie de réforme administrative générale, a approuvé plus de 30 projets spécifiques visant à réformer l'administration fédérale. Parmi eux, neuf projets devraient avoir des répercussions sur l'administration dans son ensemble (projets transversaux) tandis que 24 projets départementaux sont destinés à améliorer les structures et processus de l'administration fédérale. Le principal objectif de la réforme est de consolider les orientations générales, de retirer des tâches administratives au Conseil fédéral et d'accroître l'efficacité de l'administration. Bien que ce ne soit pas le motif principal de la réforme, le Parlement suisse escompte quelques économies budgétaires. Les neuf projets transversaux (PT) portent sur :

PT1 : le réexamen des structures départementales et de la gestion.

PT2 : I'optimisation de la gestion du personnel.

PT3 : la simplification et la réduction de la densité normative en matière de personnel.

PT4 : la répartition juste et claire des tâches, compétences et responsabilités entre les différents domaines.

PT5 : la simplification de l'organisation des achats (approvisionnement).

PT6 : la simplification des procédures et des documents.

PT7 : l'examen des commissions extraparlementaires.

PT8 : le regroupement des bibliothèques et des services de documentation.

PT9 : I'examen formel du droit fédéral.

185. En novembre 2006, le Département fédéral des finances (DFF), dans le cadre de la réforme de l'administration fédérale de 2005-2007, a mis en œuvre deux projets transversaux en rapport avec le domaine de la gestion des ressources humaines (GRH) : PT2, qui concerne l'optimisation de la gestion du personnel et PT3, qui concerne la simplification et la réduction de la densité normative en matière de personnel. Ces projets devraient donner à la Confédération, en tant qu'employeur, davantage de

37 Le double-emploi désigne une activité qui est réalisée dans deux services administratifs différents. 
souplesse pour définir et modifier les relations de travail avec ses employés. De plus, ces projets ont pour but de rendre le service public plus attractif et plus compétitif dans le cadre du vaste marché du travail.

\section{Quels sont les moteurs de la réforme?}

186. La dernière réforme de l'administration remonte à la fin des années quatre-vingt-dix. Cependant, un environnement socio-politique plus complexe et des pressions financières ont motivé la réforme. En effet, le besoin de consolider le budget, de modifier en partie des procédures rigides, compliquées, coûteuses et laborieuses et le risque d'une baisse de la compétitivité sont les principales forces à l'origine de la réforme de la GRH dans le secteur public suisse. Les dispositions actuelles empêchent la Confédération d'adapter ses pratiques de GRH aux défis d'une compétitivité croissante sur le vaste marché du travail. Avec les pratiques traditionnelles de l'administration, l'élimination des acteurs médiocres ou la modification unilatérale des contrats de travail est laborieuse et compliquée même si ces mesures sont pleinement justifiées. Selon le DFF, la loi sur la gestion du personnel présente deux inconvénients principaux: l'énumération exhaustive des raisons pour lesquelles un contrat de travail est suspendu et le principe selon lequel la Confédération n'est pas autorisée à renvoyer un employé avant d'avoir épuisé toutes les autres solutions pour empêcher le licenciement ou d'avoir envisagé d'autres postes pouvant correspondre à ses compétences.

187. Depuis 2003, le Conseil fédéral met en œuvre une stratégie de consolidation budgétaire. Cette mesure économique s'est imposée en raison du recul des recettes fiscales, qui était dû à la chute des prix de la bourse et à la morosité économique. Le programme d'allègement budgétaire prévoit essentiellement des réductions de dépenses; cependant, les dépenses affectées à l'éducation, à la recherche et au développement des nouvelles technologies devraient augmenter. Les mesures économiques envisagent une réduction du nombre de postes et la limitation des augmentations de salaires pour accroître les économies. En fait, la réforme de l'administration fédérale envisage de réduire les dépenses et le nombre de postes de $13 \%$ (4 200 postes environ) en $2009^{38}$.

\section{Qu'est-ce qui a changé ?}

188. En novembre 2006, le DFF a entrepris la mise en œuvre du PT2 sur l'optimisation de la gestion du personnel dont le but est de créer un système de gestion du personnel rationnel centré sur les résultats. Il assigne au Bureau fédéral du personnel (OFPER) le rôle d'organisme central chargé de la formulation des politiques relatives à la $\mathrm{GRH}$; il octroie aux ministères et bureaux opérationnels davantage de souplesse pour gérer leur personnel en fonction de leurs besoins spécifiques et des objectifs stratégiques généraux. De plus, le PT2 attribue à chaque niveau administratif (Confédération, ministère et bureau) des tâches, compétences et responsabilités spécifiques en matière de gestion du personnel, y compris la formation de leur personnel. Quant à l'OFPER, il est responsable de la formation des cadres supérieurs, des spécialistes du personnel, des apprentis et des formateurs. D'une façon générale, le réexamen et la redistribution des tâches, des compétences et des responsabilités en matière de gestion du personnel ont pour but de simplifier et d'accroître l'efficacité de la gestion du personnel. Selon le DFF, après une année de réforme le PT2 a contribué non seulement à réduire le nombre de postes et à éliminer les tâches inutiles, mais aussi à instaurer une culture plus orientée vers les usagers dans le service public. L'efficacité et la sensibilisation aux résultats souhaités se sont accrues

38 Fin 2007, les autorités suisses ont déjà supprimé 2600 postes dans le service public. 
et, par-dessus tout, on prend de plus en plus conscience du rôle de la GRH dans l'administration publique. $^{39}$

189. Les procédures de gestion du personnel de la Confédération sont jugées plus laborieuses et moins efficaces que celles du secteur privé. Partant, le PT3 sur la simplification et la réduction de la densité normative en matière de personnel vise à créer une nouvelle relation de travail de façon à instaurer davantage de souplesse sur le plan de la gestion du personnel. Le DFF, en coopération avec les syndicats, a réalisé une étude comparative sur les pratiques de GRH dans différents bureaux fédéraux, y compris le Bureau fédéral du personnel. Ses conclusions ont montré que les procédures actuelles sont laborieuses et exigeantes en ressources. Le Conseil fédéral a par conséquent demandé une révision de la loi sur la gestion du personnel afin de simplifier les formalités et procédures de prise de décision en matière de gestion du personnel. En 2008, le DFF devrait mener un processus de consultation sur la loi auprès des groupes d'intérêt, des institutions publiques, et des partis politiques entre autres, avant de soumettre les changements à l'approbation du Parlement.

190. Le PT2 et le PT3 constituent une réorientation des pratiques, processus et procédures de GRH qui a pour but d'accroître la compétitivité, l'efficience et l'efficacité. Pour faire évoluer la culture administrative du service publique, il ne suffit pas de veiller aux procédures liées à la réalisation des objectifs budgétaires. Le tableau 5 décrit la situation actuelle du système de GRH suisse et dépeint la vision pour 2015 dans ce domaine.

Tableau 5. Caractéristiques du système de GRH suisse

\begin{tabular}{|c|c|c|}
\hline \multicolumn{2}{|c|}{ Situation initiale du système de GRH } & \multirow{2}{*}{ Vision pour 2015 en matière de GRH } \\
\hline Atouts & Faiblesses & \\
\hline $\begin{array}{l}\text { - Base normative claire } \\
\text { - Instruments (paie, gestion par } \\
\text { objectifs etc.) } \\
\text { - Systèmes informatiques pour la } \\
\text { gestion du personnel }\end{array}$ & $\begin{array}{l}\text { - Pas de rôles clairement définis en } \\
\text { matière de gestion du personnel } \\
\text { - Même tâches à différents niveaux } \\
\text { administratifs (« double-emplois " } \\
\text { - Normes de prestation de services } \\
\text { et de qualité }\end{array}$ & $\begin{array}{l}\text { - L'Administration fédérale en tant } \\
\text { qu'employeur attractif, } \\
\text { compétitif. } \\
\text { - Reconnaissance des compétences } \\
\text { du personnel fédéral. } \\
\text { - Relations de travail reposant sur le } \\
\text { respect, la loyauté et l'estime de } \\
\text { soi. }\end{array}$ \\
\hline
\end{tabular}

Source : OCDE (GOV) sur la base de l'intervention du délégué suisse lors de la réunion du Groupe de travail sur l'emploi et la gestion publics les 28 et 29 novembre 2007 au siège de l'OCDE.

191. Le PT2 et le PT3 visent clairement à modifier l'image de l'administration fédérale pour la rendre plus attrayante aux yeux des demandeurs d'emplois. D'une façon générale, les autorités suisses cherchent à accroître la compétitivité du service public sur le marché du travail pour attirer et retenir le personnel hautement qualifié en modifiant les relations de travail et en reconnaissant les bons éléments. Le PT2 a été déployé rapidement en tant que projet susceptible de donner des résultats rapides et majeurs. II implique l'adoption d'une méthode orientée vers les performances, de techniques de gestion des performances et une délégation d'autorité aux ministères et agences opérationnels en matière de GRH.

39 Pour de plus amples informations voir DFF (2007b). 


\section{Comment le changement est-il géré ?}

192. L'une des caractéristiques les plus importantes du processus de réforme dans le secteur public suisse est le fait d'avoir élaboré une vision pour son système de GRH. ${ }^{40}$ Tous les départements et la Chancellerie fédérale se sont mis d'accord sur une stratégie qui donne de la cohérence et une orientation aux efforts de réforme. Les objectifs des projets transversaux et départementaux sont de faire de l'administration fédérale un employeur attractif et compétitif qui reconnaît les bons éléments et pour qui les relations de travail reposent sur le respect, la loyauté et l'estime de soi. Le fait même que les sept ministères ainsi que les représentants des employés se soient mis d'accord sur cette vision octroie aux projets de réforme un appui politique, en créant une coalition directrice qui soutient, défend et explique aux fonctionnaires et aux citoyens pourquoi le changement est nécessaire.

193. L'existence de plusieurs projets de réforme transversaux et départementaux fait qu'une direction centrale est nécessaire. Une équipe de projet centrale ainsi qu'une commission appelée "Ausschuss" composée de trois ministres et du Chancelier fédéral ont été constituées pour accompagner l'ensemble de la réforme. L'OFPER est chargé de diriger le PT2 et le PT3. Cependant, le Conseil fédéral, composé des sept ministres, demeure entièrement responsable de l'ensemble de la réforme administrative. Dans le même temps, le Conseil fédéral se présente comme le dirigeant de la réforme. De plus, le processus de réforme habilite chaque ministère et chaque agence à mettre en œuvre la vision et à éliminer les obstacles à la mise en œuvre du changement. On peut également avancer que le PT2 et le PT3 sont des exemples de cohérence de l'action en matière de gestion du changement. En effet, il s'agit de projets spécifiques qui vont dans le sens de stratégies de réforme plus générales, dans ce cas la réforme de l'administration fédérale et les mesures économiques générales. La cohérence permet d'éviter la confusion et l'apparition d'opinions négatives en rendant les objectifs de réforme intelligibles pour l'ensemble des parties prenantes.

194. Pour introduire le changement, il faut avoir les moyens de le faire, que ce soit en temps, en connaissances, en finances et en ressources humaines. Dans ce cas, les dépenses engendrées par la mise en œuvre des projets transversaux sont financées par l'ensemble des départements et par la Chancellerie fédérale, tandis que les coûts des projets départementaux sont couverts par le budget ordinaire de chaque département. Les réformes sont conçues et mises en œuvre par le même personnel de l'administration fédérale. La délégation de responsabilités en matière de gestion du personnel aux cadres opérationnels s'est traduite par une nouvelle répartition des tâches dans le domaine de la GRH. Les cadres devront être formés avant de prendre leurs nouvelles fonctions au sein du secteur public.

195. Le temps ne semble pas avoir été un obstacle à la réforme, car le processus de changement a été constant. Par exemple, la nouvelle loi relative à la gestion du personnel a été introduite en 2002, le processus de consolidation du budget a commencé en 2003, et la réforme du service public en 2005. Cela a permis de fixer des objectifs à moyen et long terme, tous orientés vers transformation de l'image du service public, en particulier en matière de GRH, d'ici 2015.

40 Pour de plus amples informations voir DFF (2007a). 


\section{En conclusion - quels sont les enseignements pour l'avenir ?}

196. Selon le DFF, fin 2007, les objectifs de la réforme de l'administration ont été atteints. ${ }^{41}$ L'expérience de la réforme suisse, en ce qui concerne le PT2 en particulier, permet de tirer des enseignements en matière de gestion du changement dans l'administration :

- La formation d'une vision d'avenir est indispensable pour donner une orientation aux efforts de réforme en facilitant la mise en place de stratégies visant à atteindre les résultats souhaités.

- La mise en place d'une coalition directrice assure le leadership et le dialogue qu'il faut pour améliorer l'acceptation et combattre la résistance probable au changement.

- La cohérence de l'action est nécessaire pour éviter les situations confuses et augmenter les chances de réussite.

- Des objectifs à moyen et long terme augmentent les possibilités que le changement se produise car ils laissent aux réformes le temps de mûrir, d'apporter des améliorations et d'être comprises par tous les acteurs concernés.

41 Pour de plus amples informations voir le communiqué du DFF du 14 décembre 2007 diffusé par les médias. 


\section{Interventions à la réunion du Groupe de travail sur l'emploi et la gestion publics 28-29 novembre 2007, siège de l'OCDE, Paris}

\begin{tabular}{lll}
\hline Veikko Liuksia & $\begin{array}{l}\text { Directeur de la politique du } \\
\text { personnel au Ministère des } \\
\text { finances, Finlande }\end{array}$ & $\begin{array}{l}\text { La gestion du changement dans le } \\
\text { secteur public finlandais }\end{array}$ \\
Françoise Waintrop & $\begin{array}{l}\text { Direction générale de la fonction } \\
\text { publique, France }\end{array}$ & $\begin{array}{l}\text { La LOLF ou comment accompagner un } \\
\text { changement de culture }\end{array}$ \\
Francesco Verbaro & $\begin{array}{l}\text { Directeur du bureau de la } \\
\text { fonction publique, Italie }\end{array}$ & $\begin{array}{l}\text { La gestion du changement dans le } \\
\text { secteur public }\end{array}$ \\
Joana Ramos & $\begin{array}{l}\text { Direction générale de } \\
\text { l'administration et de la fonction } \\
\text { publique, Portugal }\end{array}$ & $\begin{array}{l}\text { Les réformes de l'administration } \\
\text { publique portugaise - points de } \\
\text { changement }\end{array}$ \\
Emilio Viciana Duro & $\begin{array}{l}\text { Ministère des administrations } \\
\text { publiques, Espagne }\end{array}$ & $\begin{array}{l}\text { La gestion du changement dans le } \\
\text { secteur public espagnol : la mise en } \\
\text { œuvre du Statut de base de l'employé } \\
\text { public }\end{array}$ \\
& $\begin{array}{l}\text { Département fédéral des } \\
\text { finances, Suisse }\end{array}$ & $\begin{array}{l}\text { La politique du personnel de la } \\
\text { Confédération dans une période de } \\
\text { changement : projets-clés au sein de } \\
\text { l'Office fédéral du personnel }\end{array}$ \\
\hline Thierry Borel & &
\end{tabular}




\section{Bibliographie}

Baulcomb, J.S. (2003), " Management of Change through Force Field Analysis », Journal of Nursing Management, 11, Blackwell Publishing Ltd., pp. 275-280.

Brunsson, N. (2006), "Administrative reform as routines ", Scandinavian Journal of Management, Vol.22, 3ème édition, Elsevier Science Ltd., pp.243-252.

Butler, M. (2003), « Managing from the Inside Out: Drawing on 'Receptivity' to Explain Variation in Strategy Implementation ", British Journal of Management, Vol. 14, édition spéciale, Blackwell Publishing Ltd., pp. S47-S60.

Christensen, T. et P. Laegreid (2007), " The Whole-of-Government Approach to Public Sector Reform », Public Administration Review, novembre-décembre, Vol. 6, 6ème édition, Blackwell Publishing Ltd, pp.1059 - 1066.

Dipartimento della Funzione Pubblica. Direttiva del Ministro per le Riforme e le Innovazioni nella Pubblica Amministrazione per una Pubblica Amministrazione di Qualitá.

Dipartimento della Funzione Pubblica. Ufficio per il Personale delle Pubbliche Amministrazioni. Circolare n.3, 2 maggio 2006.

Dipartimento della Funzione Pubblica. Per una Nuova Qualitá del Servizi e delle Funzioni Pubbliche. Memorandum d'intesa su lavoro pubblico e riorganizzazione delle Amministrazioni Pubbliche. 18 gennaio 2007.

Doig, A. et J. Wilson (1998), « What Price New Public Management? », The Political Quarterly Publishing Co. Ltd., pp. 267-276.

Doyle, M. (2002), "Selecting Managers for Transformational Change ", Human Resource Management Journal, Vol.12, No. 1, pp. 3-16.

Driscoll A. et J. Morris (2001), "Stepping out: Rhetorical Devices and Culture Change Management in the UK Civil Service ", Public Administration, Vol. 79, No.4, pp. $803-824$.

Drucker. P. (1995), Managing in a Time of Great Change, Penguin Books Ltd., New York, États-Unis.

Dussauge, M (2007), « Paradojas de la Reforma Adminsitrativa en México », Buen Gobierno, No. 2, pp.28 $-42$.

Dyer, G. (2006), Future Tense: the coming world order, Mackays of Chatham, Londres, Angleterre.

Evans, M. (dir. pub.) (2004), Policy Transfer in Global Perspective, Ashgate Ltd., Aldershot, Angleterre.

Ferlie, E., J. Hartley, et S. Martin, (2003), « Changing Public Service Organizations: Current Perspectives and Future Prospects ", British Journal of Management, Vol. 14, édition spéciale, Blackwell Publishing Ltd., pp. S1-S14.

Garvin, D. A. (1993), « Building a Learning Organisation », Harvard Business Review, 71 (4), pp. 78-91.

Giddens, A. (2000), The Third Way and its Critics, Polity Press, Cambridge, Angleterre.

Hushion, N. (1999), "Managing Change or Navigating through Turbulent Times ", Museum International, No.202, (Vol. 51, No.2), pp. 44-49.

Ivanova, V. and M. Evans (2004), " Policy Transfer in a Transition State: the case of the local government reform in the Ukraine », in M. Evans (dir. pub.), Policy Transfer in Global Perspective, Ashgate Ltd., Aldershot, Angleterre. 
Kettl, D. (2000), "The Transformation of Governance: Globalization, Devolution, and the Role of Government ", Public Administration Review, Vol.60, No. 6, pp.488-497.

Kotter, J.P. (1995), « Leading Change: Why Transformation Efforts Fail », Harvard Business Review, marsavril, pp. 59-67

Ladi, S. (2004), « Environmental Policy Transfer in Germany and Greece », in M. Evans (dir. pub.), Policy Transfer in Global Perspective, Ashgate Ltd., Aldershot, Angleterre.

Lana, X. et M. Evans (2004), « Policy Transfer between Developing Countries: The Transfer of the BolsaEscola Programme to Ecuador ", in M. Evans (dir. pub.), Policy Transfer in Global Perspective, Ashgate Ltd., Aldershot, Angleterre.

McDonald, R. et S. Harrison (2004), " Autonomy and Modernisation: the management of change in an English primary trust ", Health and Social Care in the Community, 12(3), Blackwell Publishing Ltd., pp. 194201.

OCDE (2001), Le secteur public au XXlème siècle : repenser le leadership, OCDE, Paris.

OCDE (2005), Moderniser l'État : La route à suivre, OCDE, Paris.

OCDE (2006), Renforcer la confiance dans l'action publique, OCDE, Paris.

OCDE (2007a), Comparative Studies for Better Governance in Asian Countries, OCDE, Séoul.

OCDE (2007b), « Making Reform Happen », supplément de l'Observateur de l'OCDE, septembre, OCDE, Paris.

OCDE (2007c), Vieillissement et service public : Le défi des ressources humaines, OCDE, Paris.

Oliver, M. J. et H. Pemberton (2004), « Learning and Change in $20^{\text {th }}$-Century British Economic Policy », Governance, Vol.17, No. 3, pp.415-441.

Peters, G. B. (1997), « Policy Transfer between Governments: The Case of Administrative Reforms », West European Politics, Vol. 20, No. 4, pp. 71-88.

Pettigrew, A. (1997), "What is a processual analysis? ", Scandinavian Journal of Management, Vol.13, No. 4, Elsevier Science Ltd., pp.337-348.

Pollitt, C. (2003), The Essential Public Manager, Open University Press, Berkshire, Angleterre.

Pollitt, C. et G. Bouckaert, (2004), Public Management Reform: a comparative analysis, Oxford University Press, Oxford, Angleterre.

Pollitt, C. et H. Summa, (1997), "Trajectories of Reform: public management change in four countries ", Public Money and Management, 17 (1) pp.7 - 18.

Pressman, J. et A. Wildavsky (1973) Implementation: How great expectations in Washington are dashed in Oakland, Berkeley, University of California Press, Los Angeles.

Reed, M. (1996), "Rediscovering Hegel: the new historicism in organisation and management studies », Journal of Management Studies, No. 33, pp. $139-158$.

Rhodes, R.A.W. (1999), Understanding Governance: policy networks, governance, reflexivity and accountability, Open University Press, Buckingham, Angleterre.

Rose, R. (2005), Learning from Comparative Public Policy: a practical guide, Routledge, Oxon.

Sabatier, P. (1991), "Toward Better Theories of the Policy Process », Political Science and Politics, pp. 147-156.

Simon, H. (1997), Administrative Behaviour, Free Press, États-Unis. 
Skinner, D. (2004), «Evaluation and Change Management: rhetoric and reality », Human Resource Management Journal, Vol. 14, No. 3, pp. 5-19.

Stoker, G. (2006), « Explaining Political Disenchantment: finding pathways to democratic renewal », The Political Quarterly, Vol. 77, No.2, Blackwell Publishing Ltd., pp. 184-194.

Street, A. (2004), « Policy Transfer in Kyrgyzstan: the case of the general practice fundholding », in M. Evans (dir. pub.), Policy Transfer in Global Perspective, Ashgate Ltd., Aldershot, Angleterre.

Sullivan, H. et M. Stewart (2006), "Who Owns the Theory of Change? ", Evaluation, Vol. 12(2), SAGE Publications, pp.179-199.

Weissert, C. et M. Goggin (2002) « Nonincremental Policy Change: Lessons from Michigan's Medicaid Managed Care Initiative ", Public Administration Review, Vol. 62, No. 2, pp. $206-216$.

Wallis, J. et Dollery, B. (2001), « Understanding Cultural Changes in an Economic Control Agency: the New Zealand Treasury ", Journal of Public Policy, 21, 2, pp.191-212.

Waugh, W. L. (2002), « Valuing Public Participation in Policy Making ", Public Administration Review, Vol, 62, No.3, pp.379-382.

Whiteley, A. (1995), Managing Change: a core values approach, Macmillan Ltd., Australie.

\section{Références Internet}

Chronologie: préparation et mise en place de la réforme.

www.ladocumentationfrancaise.fr/dossiers/lolf/chronologie.shtml

Département fédéral des finances, DFF. Révision du droit du personnel et réorganisation de la gestion du personnel au sein de la Confédération.

www.efd.admin.ch/

Département fédéral des finances, DFF. Réforme de l'administration fédérale 05-07.

www.efd.admin.ch/

Département fédéral des finances. Programme d'allégement budgétaire de 3,3 à 3,4 milliards mis au point - mesures fiscales complémentaires à l'étude.

www.edf.admin.ch/

Département fédéral des finances, DFF. (2007a) Vision RH 2015/mission RH / orientations stratégiques, 8 janvier 2007, Berne.

Département fédéral des finances, DFF. (2007b) Réforme de l'administration : résultats des projets transversaux. Documentation de base, 14 décembre 2007, Berne.

Département fédéral des finances, DFF (2007). Réforme de l'administration réussie - projets au stade de la mise en oeuvre, communiqué dans les médias du 14 décembre 2007.

www.edf.admin.ch/00468/index.html?lang=fr 
Direcção-Geral da Administração Pública (2004), Avaliação do desempenho dos recursos humanos da Administração Pública. Aplicação do novo sistema de avaliação.

www.dgap.gov.pt/3rjur/circulares/2004/circ_1_DGAP_2004.htm

Direcção-Geral da Administração Pública, Sistema Integrado de Avaliação do Desempenho da Administração Pública.

www.dgap.gov.pt

Goman, C. K. (2000), The Biggest Mistakes in Managing Change, No.506 from Innovative Leader Volume 9, Number 12, www.winstonbrill.com/bril001/html/article_index/articles/501-550/article506. Harding, P. (2004), Managing Change: a guide on how to manage change in an organisation, www.oursouthwest.com.

Institut de la gestion publique et du développement économique (2002) Public Management Outlook, publication de l'IGPDE $n^{\circ} 3$, septembre.

www.comite-histoire.minefi.gouv.fr

Ley 7/2007, de 12 de abril, del Estatuto Básico del Empleado Público.

http://noticias.juridicas.com/base_datos/Admin/17-2007.html

Lorenzi, N. et R. Riley (2000), Managing Change: an overview, www.pubmedcentral.nih.gov/articlerender.fcgi?artid=61464.

Ministerio de las Administraciones Públicas (MAP)

www.map.es/prensa/notas_de_prensa/2007/05/2007_05_13.html

Nickols, F. (2006), Change Management 101: a premier, http://home.att.net/ nickols/change.htm. Reh, F. J. (2007), Managing Change: managing people's fear, http://management.about.com/cs/people/a/MngChng092302.htm.

\section{Entretiens}

Entretien avec M. Asko Lindqvist, directeur du programme Finwin, Finlande, le 10 décembre 2007.

Entretien avec Mlle Joana Ramos, Direction générale de l'administration et de la fonction publique, Portugal, le 10 décembre 2007. 